\title{
Medical Species Used in Russia for the Management of Diabetes and Related Disorders
}

\begin{abstract}
Alexander N. Shikov ${ }^{1 *}$, Igor A. Narkevich ${ }^{1}$, Alexandra V. Akamova ${ }^{1}$, Oksana D. Nemyatykh ${ }^{1}$, Elena V. Flisyuk ${ }^{1}$, Vladimir G. Luzhanin ${ }^{2}$, Mariia N. Povydysh ${ }^{1}$, Iuliia V. Mikhailova ${ }^{1}$ and Olga N. Pozharitskaya ${ }^{3}$
\end{abstract}

${ }^{1}$ Saint-Petersburg State Chemical Pharmaceutical University, Saint-Petersburg, Russia, ${ }^{2}$ Perm State Pharmaceutical Academy, Perm, Russia, ${ }^{3}$ Murmansk Marine Biological Institute of the Russian Academy of Sciences (MMBI RAS), Murmansk, Russia

Background: Polyherbal mixtures called "medical species" are part of traditional and officinal medicine in Russia. This review aimed to analyze medical species used in Russia for the treatment of diabetes and related disorders. The information relevant to medical species, diabetes, and obesity was collected from local libraries, the online service E-library.ru, and Google Scholar. The prediction of the antidiabetic activity for the principal compounds identified in plants was performed using the free web resource PASS Online.

Results: We collected and analyzed information about the compositions, specificities of use, and posology of 227 medical species. The medical species represent mixtures of 2-15 plants, while the most frequently mentioned in the literature are species comprising 3-6 plants. The top 10 plants among the 158 mentioned in the literature include Vaccinium myrtillus L., Phaseolus vulgaris L., Taraxacum campylodes G.E. Haglund., Urtica dioica L., Rosa spp., Hypericum spp., Galega officinalis L., Mentha $\times$ piperita L., Arctium spp, and Fragaria vesca $L$. The leading binary combination found in medical species comprises the leaves of $V$. myrtillus and pericarp of $P$. vulgaris; leaves of $V$. myrtillus and leaves of $U$. dioica; and leaves of $V$. myrtillus and aerial parts of $G$. officinalis. In triple combinations, in addition to the above-mentioned components, the roots of $T$. campylodes are often used. These combinations can be regarded as basic mixtures. Other plants are added to improve the efficacy, treat associated disorders, improve gastrointestinal function, prevent allergic reactions, etc. Meanwhile, an increase in plants in the mixture necessitates advanced techniques for quality control. A feature of medical species in Russia is the addition of fresh juices, birch sap, seaweeds, and adaptogenic plants. Modern studies of the mechanisms of action and predicted activities of the principal compounds from medicinal plants support the rationality of polyherbal mixtures. Nevertheless, the mechanisms are not well studied and reported due to the limited number of compounds. Further investigations with calculations of synergistic or additive indices are important for strengthening the scientific fundamentals for the wider use of medical species in the therapy of diabetes. Two medical species, "Arfazetin" (7 medicinal plants) and "Myrphasinum" (12 medicinal plants), are approved for use in officinal medicine. The efficacy of these species was confirmed in several in vivo experiments and clinical trials. 
According to modern regulatory rules, additional experiments and clinical trials are required for more detailed investigations of the mechanisms of action and confirmation of efficacy.

Conclusion: We believe that the scientifically based utilization of rich plant resources and knowledge of Russian herbal medicine can significantly contribute to the local economy as well as to the sectors seeking natural healing products.

Keywords: polyherbal mixture, herbal medicine, obesity, blood glucose, binary combination, triple combination, mechanisms of activity, synergy

\section{INTRODUCTION}

Disorders of carbohydrate and lipid metabolism predispose individuals to diseases of the endocrine system, particularly diabetes. The rapid increase in patients with diabetes is one of the gravest and fastest-growing public health problems in the world. About 463 million people currently suffer from diabetes globally (IDF, 2019), of whom over 60 million were in European countries (Timmis et al., 2020), 34.2 million in the US (Centers for Disease Control and Prevention, 2020), and 4.5 million in Russia in 2017 (Dedov et al., 2018).

The mean cost of the development of a new drug from concept to market is considered to range from $\$ 314$ million to $\$ 2.8$ billion (Wouters et al., 2020). According to recent literature data, $50-70 \%$ of all the small-molecule therapeutics in clinical use today trace their origins to natural products (Newman and Cragg, 2020). Medicines derived from natural sources exhibit greater ranges of structural and physicochemical features that have been tailored through evolution for selective binding to functional macromolecules of the human body (Stratton et al., 2015). The intensive exploration of natural resources and utilization of the knowledge of traditional medicine provides an opportunity to reduce the time needed for development and keep costs reasonably low.

About four billion people around the world believe that, as "natural" products, herbal medicinal products (HMP) are "safe" or "safer" than conventional drugs and have turned to phytotherapeutics (Ekor, 2014). For centuries, Russia has been regarded a "herbophilious" society in which plants have been used as one of the primary foods and for the treatment of different diseases (Shikov et al., 2017). It is estimated that $58-60 \%$ of the population of Russia relies on HMP for the prophylaxis or treatment of different diseases (Shikov et al., 2011; Sammons et al., 2016). HMP currently makes up $20 \%$ of the Russian market for drugs (Akamova et al., 2017).

Medicinal plants have been effectively used for the treatment of diabetes in different systems of traditional as well as officinal medicine (Anzar, 2013; Shikov et al., 2014; Suzuki et al., 2017; Xiao and Luo, 2018; Okovitiy et al., 2018; Skalli et al., 2019; Salehi et al., 2019). The philosophy "one disease, one target, one drug" oversimplifies the mechanisms of disease and is becoming increasingly inefficient (Ulrich-Merzenich, 2014; Panossian et al., 2018; Shikov et al., 2018). Due to its multifactorial etiology, the holistic treatment of diabetes requires multipathway understanding and multi-targeting approaches.
Modern network pharmacology studies emphasize the importance of the network-targeting, multicomponent therapy used in traditional Indian systems of medicine (Mukherjee et al., 2018; Banerjee et al., 2019), traditional Chinese medicine (Li et al., 2014; Xue et al., 2019), Kampo (Suzuki et al., 2017), etc.

Multicomponent or polyherbal mixtures for the treatment of diabetes are presented in different systems of Eastern traditional medicine (Namdul et al., 2001; Sato, 2004; Tong et al., 2012; Malgaonkar et al., 2016; Ghadge and Kuvalekar, 2017; Suzuki et al., 2017; Xu et al., 2019) as well as in European herbal medicine (Madić et al., 2021). Such traditional formulations include carefully selected leaves, stems, flowers, roots, seeds, sometimes minerals, and animal products. The main goal of complicated mixtures is to increase therapeutic efficacy and minimize toxicity.

Russian herbal medicine has adopted Eastern philosophy and a Western pragmatic approach. Herbal medicine in Russia is part of officinal medicine. According to the 14th edition of the State Pharmacopoeia of the Russian Federation, which became effective in 2018, an HMP has been defined as "a medicinal product manufactured or prepared from one kind of medicinal plant material or several kinds of raw materials and marketed in consumer-ready packaging form" (The State Pharmacopoeia of Russian Federation, 2018). The general monograph (OFS.1.4.1.0020.15) is devoted to polyherbal mixtures, which are defined with the specific term "medical species". A "medical species" is a formulation representing a mixture of two or more types of integral, cut, or powdered medicinal plant materials, sometimes with the addition of mineral, synthetic, plant, or animal-derived substances. Medical species are used for the preparation of aqueous decoctions/infusions, occasionally in pure form as powders, powders for insufflation or ingestion, etc. (The State Pharmacopoeia of Russian Federation, 2018). Medical species have been known in Russia for centuries and were documented in the first herbalist manuscripts (Zmeev, 1896; Shikov et al., 2021). Apparently, due to their efficacy, the medical species were adopted from traditional medicine in officinal and were monographed in the first Russian military field “Pharmacopoeia Castrensis Rossica., 1765". Thereafter, medical species were embodied in all the following pharmacopoeias of Russia.

The polyherbal mixtures used in Eastern systems of traditional medicine have gained global popularity, and several new medicinal products are being marketed in different countries. However, the potential of the medical species used in Russia 
remains little known and underestimated. This review aimed to analyze the medical species used in Russia for the treatment of diabetes and related disorders and enable a better understanding of the rationality of plant combinations.

Information on medical species was collected from the Pharmacopoeias of Russia (I-VI editions), Pharmacopoeias of USSR (VII-XI editions), and online State Register of Medicinal Preparations of the Russian Federation (2021). Guided by the keywords "traditional medicine + diabetes", "phytotherapy + diabetes", "traditional medicine + obesity", and "phytotherapy + obesity", we systematically searched the literature in library catalogs, on the online service E-library.ru, and on Google Scholar. Next, the publications were screened using the keyword combination "medical species". Through this approach, 75 medical (herbalist) books were found. Some medical species were mentioned in different books. We provide several references for the same medical species in Table 1.

\section{RESULTS AND DISCUSSION}

For centuries, medicinal plants have been used in Russia for the management of diabetes and related disorders (Zmeev, 1896; Turova and Sapozhnikova, 1989; Minaeva, 1991; Protasenya and Vasilenko, 1992; Nazina, 2007; Chekina et al., 2010; Korsun et al., 2016; Povydysh et al., 2018). The focus of the current paper is on medical species used for the therapy of diabetes and related diseases in traditional and officinal Russian medicine.

\section{The Characteristics of the Composition of Medical Species, Their Preparation and Posology}

We collected information about the composition, specificity of preparation, and posology of 227 species (Table 1). The majority of the species (148) are suggested for the treatment of diabetes; 37 species are recommended for the management of obesity, and eight species are indicated for lowering blood glucose. Several species are recommended for specific cases when diabetes is accompanied by impotence in men (5 species), diabetes accompanied by liver and gallbladder diseases (3 species), diabetes accompanied by gastritis (2 species), etc. Altogether, 158 plants are mentioned in medical species, among which 96 medicinal plants are monographed in the State Pharmacopoeia of the Russian Federation and used in officinal medicine (Shikov et al., 2021). A significant proportion of the plants used in medical species are native to Russian flora.

Medical species represent mixtures of $2-15$ plants. The most frequently mentioned in the literature are medical species comprising mixtures of four plants (66 species), followed by mixtures of five plants (47 species), three plants (28 species), and six plants (24 species) (Figure 1). According to some experts' opinions, the industrial-scale production of polyherbal mixtures with more than 10 medicinal plants is not rational (Kiseleva and Chauzova, 1999). Indeed, only 18 species among the 227 described contain over 10 plants (Figure 1). However, the numbers of plants in polyherbal mixtures in Ayurveda (Parasuraman et al., 2014), Kampo (Arai et al., 2020), and traditional Chinese medicine (Xutian et al., 2014) are not limited to 10. On the other hand, the species "Myrphasinum", approved as officinal medicine in Russia, includes 12 plants (Table 1). Although the idea of combining so many plants in one mixture is part of traditional medicine, the quality control of medical species becomes more complicated with each additional component due to challenges related to the specificity of each plant.

Medical species are prepared predominantly in form of infusions or decoctions. Infusions are common for soft plant parts such as aerial parts, leaves, and flowers. Decoctions are preferred for more hard barks, fruits, and roots. The recommended single doses vary from a tablespoon up to $200 \mathrm{ml}$ and depend on the pharmacological activity of the plants in the mixture.

\section{The Plants Most Frequently Used in Medical Species}

The top 10 plants in medical species used for the therapy of diabetes and related disorders (Table 1) include Vaccinium myrtillus L. (leaves in 97, shoots in 11, and fruits in 5 species), Phaseolus vulgaris L. (pericarp in 65 species), Taraxacum campylodes G.E. Haglund. (syn. Taraxacum officinale Wigg) (roots in 49 and leaves in 15 species), Urtica dioica L. (leaves in 49 and roots in 1 species), Rosa spp. (fruits in 44 species), Hypericum spp. (aerial parts in 37 and flowers in 6 species), Galega officinalis L. (aerial parts in 41 species and seeds in one species), Mentha $\times$ piperita L. (leaves in 29 and aerial parts in 11 species), Arctium spp. (roots in 34 and leaves in 2 species), and Fragaria vesca L. (leaves in 26, aerial parts in 5, and roots in 1 species). Although the main plant parts used in species are the same as those recorded in the State Pharmacopoeia of the Russian Federation, multiple parts of some plants are utilized. Particularly, aerial parts, fruits, and roots (Petroselinum crispum (Mill.) Fuss); leaves, flowers, and roots (Sambucus nigra L.); and roots and leaves (Cichorium intybus L., Rubus caesius L., and Arctium spp.) have been used.

\section{The Popular Combinations of Medicinal Plants and Rationality for Combination}

It is believed that, in medical species, several herbs work together harmoniously to achieve an ideal therapeutic effect. Modern studies on the mechanisms of activities of individual plant extracts support the rationality of empirically composed polyherbal mixtures in traditional medicine. Furthermore, we discuss the most frequent combinations of plants used in medical species in light of their mechanisms of action. The most frequently mentioned binary combinations of plants in medical species used for the treatment of diabetes are specified in Table 2.

The leading binary combination noted in medical species (Table 2) comprises the leaves of Vaccinium myrtillus L. and pericarp of Phaseolus vulgaris L. (quoted in 40 medical species). 
TABLE 1 | The list of medical species used for the management of diabetes and related disorders in Russia.

Code $^{\text {a }}$ Plant name, part used /(proportion) Method of preparation

Recommended

Indication

dosage

\section{2 plants}

2.1 Frangula alnus Mill. bark; Taraxacum campylodes G.E.Haglund. roots; (10:3)

2.2 accinium myrtillus L. leaves; Arctium spp. ${ }^{b}$ roots; (1:1)
Decoction; 1 table spoon in $200 \mathrm{ml}$ of boiling water Infusion; $10 \mathrm{~g}$ in $200 \mathrm{ml}$ of boiling water
$200 \mathrm{ml} 2-3$ times a day

Obesity

1 table spoon 3-4 times a Diabetes day before eating
Vaccinium myrtillus L. leaves;

Phaseolus vulgaris L. pericarp; (1:1)
Infusion; 1 table spoon in $200 \mathrm{ml}$ of water, boil 15 min, maceration 30 min at room temp.
$100 \mathrm{ml}$ 3-4 times a day Diabetes before eating 
TABLE 1 | (Continued) The list of medical species used for the management of diabetes and related disorders in Russia.

Code $^{\mathrm{a}}$ Plant name, part used /(proportion) Method of preparation

3.6 Frangula alnus Mill. bark; Achillea millefolium L. aerial part; Juniperus communis L. fruits; (3:2:1)

3.7 Vaccinium myrtillus L. leaves; Urtica dioica L. leaves; Sambucus nigra L. leaves; $(2: 1: 1)$ in $200 \mathrm{ml}$ boiling water

Method of preparation $\quad \begin{array}{r}\text { Recommen } \\ \text { dosag }\end{array}$
$\begin{aligned} & \text { Infusion; } 2 \text { table spoons in } \\ & 500 \mathrm{ml} \text { of boiling water }\end{aligned}$
$\begin{aligned} & \text { Decoction; } 1 \text { table spoon } \\ & \text { in } 200 \mathrm{ml} \text { boiling water }\end{aligned}$

Infusion; 1 table spoon in $400 \mathrm{ml}$ hot water

arvense $L$ aerial part Polygonum aviculare $\mathrm{L}$. aerial part; Fragaria vesca L leaves; (1:2:1)
Vaccinium myrtillus L. leaves; Urtica dioica L. leaves; Taraxacum campylodes G.E.Haglund. roots; (1: $1: 1)$
Infusion (herbal tea); $10 \mathrm{~g}$ in $200 \mathrm{ml}$ of boiling water
$100 \mathrm{ml} 3$ times a day before eating
Lowering of blood glucose level
Lowering of blood glucose level
Obesity

Lowering of blood glucose level

Reference

Lager (1991), Lager (2002), Dontsov and Dontsov (2000), Rendiuk (2006), Safonov (2016 Sokolov and Zamotaiev (1984), Matkovskaya et al. (1988), Sinyakov (1992), Sinyakov (1999); Efimov and Shcherbak (1993), Tarasenko et al. (1998), Kukes (1999), Blinov (2000), Dontsov and Dontsov (2000), Sokolov (2000), Bubenchikova et al. (2003), Podduev (2001), Onipko (2002), Davydovich et al. (2008), Bogdanova and Bashkirova (2010)

Sokolov and Zamotaiev (1984), Matkovskaya et al. (1988), Sinyakov (1992), Sinyakov (1999); Chirkov and Seryi (1993), Nikultseva (1994), Tarasenko et al. (1998), Trofimenko and Mogilny (1998), Kukes (1999), Dontsov and Dontsov (2000), Sokolov (2000), Bubenchikova et al. (2003), Turishchev (2000), Blinov (2000), Podduev (2001), Dremova et al. (2003), Turishchev (2005), Nazina (2006), Davydovich et al. (2008), Volynchenko (2003),

Ruzhenkova (2014), Maznev (2014)

Sokolov and Zamotaiev (1984), Matkovskaya et al. (1988), Sinyakov (1992), Chirkov and Seryi (1993), Efimov and Shcherbak (1993), Nikolaychuk (1997), Fedyukovich (1998), Kukes (1999), Bubenchikova et al. (2003), Turishchev (2000), Sokolov (2000), Blinov (2000), Dontsov and Dontsov (2000), Podduev (2001), Onipko (2002), Nikolaychuk and Zubitskaya (2003), Dremova et al. (2003), Brusenskaya and Kaz'min (2005), Turishchev (2005), Nazina (2006), Davydovich et al. (2008), Bogdanova and Bashkirova (2010), Pigulevskaya (2018)

Brusenskaya and Kaz'min (2005) boiling water

$100 \mathrm{ml}$ or $66 \mathrm{ml} 4$ times a Diabetes Galega officinalis L. aerial part; Urtica dioica L. leaves; $(5: 4: 3)$

3.11 Hypericum perforatum L. aerial part; Mentha $\times$ piperita $L$ leaves; Vaccinium myrtillus L. leaves; $(1: 1: 1)$

3.12 Vaccinium myrtillus L. leaves; Elymus repens(L.)rhizomes; Rubuscaesius L. roots; (250:10:2)

Infusion; 1 table spoon in $250 \mathrm{ml}$ of boiling water

Infusion; $262 \mathrm{~g}$ in $1,500 \mathrm{ml}$ of boiling water day before eating

$125 \mathrm{ml} 2$ times a day before eating

During the day instead of Diabetes water

Diabetes
Osetrov and Shreter (2001)

Osetrov (1993), Osetrov and Shreter (2001)

(Continued on following page) 
TABLE 1 | (Continued) The list of medical species used for the management of diabetes and related disorders in Russia.

Code $^{\mathrm{a}}$ Plant name, part used /(proportion) Method of preparation

3.13 Vaccinium vitis-idaea L. leaves; Ruta
graveolens L. leaves; Angelica archangelica L. roots; (5:3:2)

3.14 Arctostaphylos uva-ursi (L.) Spreng. leaves; Valeriana officinalis $L$. roots and rhizomes; Vaccinium myrtillus L. leaves; (1:1:2)

3.15 Fragaria vesca L. leaves; Cichorium intybus L. leaves; Sambucus nigra L. flowers; (2:2:1)

3.16 Vaccinium myrtillus L. leaves; Fragaria vesca L. leaves; Rubuscaesius $L$. leaves; $(1: 1: 1)$

3.17 Vaccinium myrtillus L. leaves; Phaseolus vulgaris L. pericarp; Matricaria chamomilla L. flowers; (1: 2:1)

3.18 Arctium spp. ${ }^{b}$ roots; Phaseolus vulgaris L. pericarp; Vaccinium myrtillus L. leaves; (1:1:1)

3.19 Vaccinium myrtillus L. leaves; Galega officinalis L. aerial part; Urtica dioica L. leaves; (1:1:1)
Infusion; 1 table spoon in $300 \mathrm{ml}$ of boiling water

Infusion; $40 \mathrm{~g}$ in $400 \mathrm{ml}$ of boiling water, maceration 5-6 $\mathrm{h}$ at room tempature

Infusion; $60 \mathrm{~g}$ in 1,000 ml of cold water, maceration $12 \mathrm{~h}$ at room temperature, boil $5 \mathrm{~min}$, maceration $1 \mathrm{~h}$
$200 \mathrm{ml}$ of boiling water,

boil $10 \mathrm{~min}$, maceration
$100 \mathrm{ml} 4$ times a day 20-30 min before eating

$150 \mathrm{ml} 5$ times a day $1 \mathrm{~h} \quad$ Diabetes after eating

Diabetes

Indication

Reference

dosage

$100 \mathrm{ml}$ 3-4 times a day Diabetes

$30 \mathrm{~min}$ before eating

Diabetes

$200 \mathrm{ml}$ 3-4 times a day Diabetes before eating

Diabetes

$66 \mathrm{ml} 3$ times a day

20 min before eating

$100 \mathrm{ml} 3$ times a day

20 min before eating

Diabetes

2 table spoons 3-4 times Diabetes a day 20 min before eating

\author{
Infusion; 1 table spoon in $100 \mathrm{ml}$ 2-3 times a day Diabetes \\ $300 \mathrm{ml}$ of boiling water 20 min before eating \\ Infusion; 1 table spoon in \\ $200 \mathrm{ml}$ of boiling water
}

Vaccinium myrtillus L. leaves;

Taraxacum campylodes G.E.Haglund. leaves; Galega officinalis L. aerial part; $(1: 1: 1)$

3.21 Leonurus spp. 'leaves; Fragaria vesca L. leaves; Morus alba L. leaves; (1:2:4)
$10 \mathrm{~min}$ at room tempature

\section{$200 \mathrm{ml}$ of boiling water,} 30 min at room temp.

$200 \mathrm{ml}$ of boiling water, boil 5 min, maceration $1 \mathrm{~h}$ $300 \mathrm{ml}$ of boilir (1)
Sinyakov (1992), Sinyakov (1999), Efimov and Shcherbak (1993), Tarasenko et al. (1998), Bogdanova and Bashkirova (2010)

Tarasenko et al. (1998), Podduev (2001)

Efimov and Shcherbak (1993), Nikolaychuk (1997), Rendiuk (2006), Bogdanova and Bashkirova (2010)

Efimov and Shcherbak (1993), Nikolaychuk (1997), Fedyukovich (1998), Nikolaychuk and Zubitskaya (2003), Dremova et al. (2003), Rendiuk (2006), Bogdanova and Bashkirova (2010), Pigulevskaya (2018)

Lavrenova and Lavrenov (2007)

Seredin and Sokolov (1973), Lager (1991), Lager (2002), Makhlayuk (1991), Chirkov and Seryi (1993), Dmitriev et al. (1994) Nikultseva (1994), Fedyukovich (1998), Podduev (2001), Pirogov (2008), Grechanyi (2013), Melik-Gusseinov and Rekkandt (2014), Maznev (2014) Sinyakov (1992), Sinyakov (1999), Efimov and Shcherbak (1993), Nikultseva (1994), Nikolaychuk (1997), Trofimenko and Mogilny (1998), Fedyukovich (1998), Blinov (2000), Onipko (2002), Nikolaychuk and Zubitskaya (2003), Nazina (2006), Bogdanova and Bashkirova (2010), Maznev (2014) Sinyakov (1992), Sinyakov (1999), Trofimenko and Mogilny (1998), Blinov (2000), Nazina (2006)

Sinyakov (1992), Sinyakov (1999), Nikultseva (1994), Nikolaychuk (1997), Tarasenko et al. (1998), Trofimenko and Mogilny (1998), Fedyukovich (1998), Blinov (2000), Podduev (2001), Dremova et al. (2003), Kiyanova (2005), Nazina (2006), Davydovich et al. (2008), Bogdanova and Bashkirova (2010), Maznev (2014) (Continued on following page) 
TABLE 1 | (Continued) The list of medical species used for the management of diabetes and related disorders in Russia.

Code $^{\mathrm{a}}$ Plant name, part used /(proportion) Method of preparation

Vaccinium myrtillus L. leaves; Inula helenium L. roots; Polygonum aviculare L. aerial part; $(1: 1: 1)$

3.23 Vaccinium myrtillus L. leaves; Taraxacum campylodes G.E.Haglund. leaves; Artemisia vulgarisL., aerial part; $(5: 5: 4)$

3.24 Vaccinium myrtillus L. leaves; Phaseolus vulgaris L. pericarp; Mentha $\times$ piperita L. leaves; (1:1:1)

3.25 Avena sativa L. aerial part in flowering phase; Vaccinium myrtillus L. leaves; Phaseolus vulgaris L. pericarp; (1:2:2)
Decoction; 1 table spoon in $200 \mathrm{ml}$ of water

Decoction; 1 table spoon in $300 \mathrm{ml}$ of water, boil 5 min, maceration 30 min

Infusion; 2 table spoons in $500 \mathrm{ml}$ of boiling water, maceration $30 \mathrm{~min}$ Infusion; 1 table spoon in $200 \mathrm{ml}$ of boiling water

\section{Recommended \\ dosage}

$50 \mathrm{ml} 2-3$ times a day

$100 \mathrm{ml}$ 3-4 times a day

Indication

Reference Podduev (2001)

\section{before eating}

$70 \mathrm{ml} 3$ times a day

$200 \mathrm{ml}$ 3-4 times a day before eating
Diabetes

Diabetes

Diabetes

Diabetes accompanied by impotence in men
3.26 Helichrysum arenarium (L.) Moench. flowers; Fagopyrum esculentum Moench flowers and leaves; Vaccinium myrtillus L. leaves; (1:1:2)

3.27 Urtica dioica L. leaves; Inula helenium L. roots; Sugar; (9:1:5)

3.28 Viola tricolor L. aerial part; Bidens tripartite L. aerial part; Solanum dulcamara L. aerial part; (4:4:1)
Infusion; $12 \mathrm{~g}$ in 1,000 ml of boiling water, maceration 5-6 h at room temp., then 15 min in boil water bath

Decoction; 2 table spoons in $200 \mathrm{ml}$ of water Infusion (herbal tea); 1 table spoon in $200 \mathrm{ml}$ of boiling water
$200 \mathrm{ml}$ (warm) with $10 \mathrm{~g}$ of honey 3-4 times a day before eating

$100 \mathrm{ml}$ (warm ) 3 times a Metabolism improving day before eating

4 table spoon 3-4 times a day
Diabetes accompanied by impotence in men

King's evil, metabolic disorder
Chirkov and Seryi (1993), Kukes (1999), Bubenchikova et al. (2003), Dremova et al. (2003)

Podduev (2001)

Sklyarevsky and Gubanov (1989), Efimov and Shcherbak (1993), Blinov (2000); Orlova, (2001), Dremova et al. (2003), Brusenskaya and Kaz'min (2005), Kiyanova (2005), Rendiuk (2006), Davydovich et al. (2008), Bogdanova and Bashkirova (2010)

Brusenskaya and Kaz'min (2005)

Chirkov and Seryi (1993)

Chirkov and Seryi (1993)

\section{4 plants}

4.1 Mentha $\times$ piperita L. leaves; Foeniculum vulgare Mill. fruits; Matricaria chamomilla L. flowers; Tilia cordata Mill. flowers; (4:3:3:3)

4.2 Levisticum officinale W.D.J.Koch roots Juniperus communis L. fruits; Phaeophyceae (Cystoseira barbata (Stackh.) C.Agardh) thallus; Achillea millefolium L. aerial part; (1:1:1:1)

4.3 Ononis spinosa L. roots; Persicaria hydropiper (L.) Delarbre aerial part; Foeniculum vulgare Mill. fruits; Alchemilla xanthoch/ora Rothm. roots and aerial part; $(6: 1: 1: 1)$

4.4 Artemisia absinthium L., aerial part; Salvia officinalis L. leaves; Rosmarinus officinalisL.leaves; Prunus spinosaL. flowers; (1:1:1:1)

4.5 Frangula alnus Mill. bark; Taraxacum campylodes G.E.Haglund.roots; Petroselinum crispum (Mill.) Fuss fruits; Foeniculum vulgare Mill. fruits; (3:1:1:1)

4.6 Apium graveolens L. leaves; Phaseolus vulgaris L. pericarp; Humulus lupulus L. fruits; Pastinaca sativa L. root; (4:4:3:1)

4.7 Nasturtium officinaleR.Br.aerial part; Morus nigraL.leaves; Urtica dioica L. leaves; Phaseolus vulgaris L. pericarp; (1:1:1:1)
Infusion; $10 \mathrm{~g}$ in $200 \mathrm{ml}$ of boiling water

Decoction; 2 table spoons in $500 \mathrm{ml}$ of water 2-3 times a day

Decoction; 2 table spoons in $500 \mathrm{ml}$ of water

$100 \mathrm{ml} 4$ times a day before eating

Infusion; 3 table spoons in $500 \mathrm{ml}$ of boiling water

Infusion; $20 \mathrm{~g}$ in $400 \mathrm{ml}$ of boiling water

Herbal tea; 1 table spoon in $200 \mathrm{ml}$ of boiling water

Infusion; 1 table spoon in $300 \mathrm{ml}$ of boiling water
$400 \mathrm{ml}$ in the morning before eating

$30 \mathrm{ml} 6$ times a day

$100 \mathrm{ml} 3$ times a day before eating
Obesity

Safonov (2016)

Obesity

Chirkov and Seryi (1993)

Obesity

Chirkov and Seryi (1993)

Osetrov and Shreter (2001)

Obesity

Dontsov and Dontsov (2000), Maznev (2005), Kiyanova (2005)

Obesity and diabetes

Protasenya and Vasilenko (1992)

Diabetes

Osetrov (1993)

(Continued on following page) 
TABLE 1 | (Continued) The list of medical species used for the management of diabetes and related disorders in Russia.

Code $^{\text {a }}$ Plant name, part used /(proportion) Method of preparation $\quad \begin{gathered}\text { Recommended } \\ \text { dosage }\end{gathered}$

4.8 Phaseolus vulgaris L. pericarp; Betula $150 \mathrm{ml} 2$ times a day Diabetes

Osetrov (1993), Maznev (2014) G.E.Haglund. roots; Sinapis albaL. seeds; (8:12:4:1)

4.9 Vaccinium myrtillus L. leaves; Rubus $300 \mathrm{ml}$ of boiling water

Diabetes
caesius L. leaves; Fragaria vesca L. leaves; Rosa majalis Herrm. fruits; 1 : 1 :

Infusion; 1 table spoon in $200 \mathrm{ml}$ of boiling water. maceration $30 \mathrm{~min}$ $1: 1)$
$100 \mathrm{ml} 3$ times a day Diabetes before eating

Diastes

\subsection{Juniperus communis L. fruits; Linum usitatissimum L. seeds; Vaccinium myrtillus L. leaves; Vaccinium vitis- idaea L. leaves; (1:1:1:1) \\ 4.11 Galega officinalis L. aerial part; Urtica dioica L. leaves; Taraxacum campylodes G.E.Haglund. roots; Phaseolus vulgaris L. pericarp; (1:1: $1: 1)$ \\ 4.12 Vaccinium myrtillus L. leaves; Fragaria vesca L. leaves; Tilia cordata Mill. flowers; Verbascum densiflorum Bertol. flowers; (8:5:4:3)}

4.13 Vaccinium myrtillus L. leaves; Urtica dioica L. leaves; Taraxacum campylodes G.E.Haglund. roots; Phaseolus vulgaris L. pericarp; (1:1:1:1)
Infusion; 1 tea spoon in $200 \mathrm{ml}$ of boiling water, boil $5 \mathrm{~min}$, maceration $30 \mathrm{~min}$

Infusion; 1 table spoon in $200 \mathrm{ml}$ of boiling water

Infusion; 2 table spoons in $400 \mathrm{ml}$ of boiling water
$50 \mathrm{ml} 3$ times a day before eating

$50 \mathrm{ml} 3$ times a day

100-132 ml 3 times a day Diabetes $30 \mathrm{~min}$ before eating

Diabetes

Diabetes Diabetes 15 min before eating

\author{
(1)
}

Infusion; 2 table spoons in $500 \mathrm{ml}$ of boiling water, boil 12-15 min, maceration 30-40

Diabetes

$66 \mathrm{ml} 3$ times a day

(a)
4.14 Equisetum arvense L aerial part; Polygonum aviculare L. aerial part; Urtica dioica L. leaves; Capsella bursapastoris (L.) Medik. aerial part; (1:1:1:1)
Infusion; 2 table spoons in $500 \mathrm{ml}$ of boiling water, boil 3-5 min, maceration 30-40 min
4.15 Polygonum aviculare L. aerial part; Equisetum arvense L. aerial part; Fragaria vesca L leaves; Aralia elata (Miq.) Seem roots; (7:5:5:2)

4.16 Taraxacum campylodes G.E.Haglund. roots; Phaseolus vulgaris L. pericarp; Hypericum perforatum $L$.erial part; Vaccinium myrtillus $L$. leaves; (1:1:1:1)

4.17 Vaccinium myrtillus $L$. leaves; Phaseolus vulgaris L. pericarp; Arctium lappa L. roots; Vaccinium vitis-idaea L. leaves; (2:2:1:1)

4.18 Rubuscaesius L. leaves;Vaccinium vitis-idaea L. leaves; Primula veris $\mathrm{L}$. leaves; Galega officinalis L. aerial part; (3:3:2:4)

4.19 Centaurium erythraea Rafn aerial part; Vaccinium myrtillus L. leaves; Equisetum arvense $\mathrm{L}$ aerial part; Polygonum aviculare L. aerial part; (1:1: 1:1)
Infusion; 2 table spoons in $500 \mathrm{ml}$ of boiling water, boil 3-5 min, maceration 20-30 min

Infusion; 2 table spoons in $500 \mathrm{ml}$ of boiling water, maceration $12 \mathrm{~h}$ in

thermos

Infusion; 1 table spoon in $200 \mathrm{ml}$ of boiling water, boil 15 min, maceration $30 \mathrm{~min}$

Infusion; 1 table spoon in $300 \mathrm{ml}$ of boiling water, boil 3 min, maceration at room tempature

Infusion; 1 table spoon in $200 \mathrm{ml}$ of boiling water, boil $5 \mathrm{~min}$, maceration at room tempature
40-50 ml 3-4 times a day Diabetes $30 \mathrm{~min}$ before eating

40-50 $\mathrm{ml} \mathrm{3-4} \mathrm{times} \mathrm{a} \mathrm{day} \mathrm{Diabetes}$ 30 min before eating

$100 \mathrm{ml} 3$ times a day 30 min before eating

$200 \mathrm{ml} \mathrm{3-4} \mathrm{times} \mathrm{a} \mathrm{day}$ before eating

$100 \mathrm{ml} 3$ times a day after Diabetes eating

$200 \mathrm{ml} \mathrm{2-3}$ times a day before eating

\author{
Dan
}


TABLE 1 | (Continued) The list of medical species used for the management of diabetes and related disorders in Russia.

Code $^{\mathrm{a}}$ Plant name, part used /(proportion) Method of preparation

4.20 Arctium lappa L. roots; Cichorium intybus L. roots; Valeriana officinalis L. roots and rhizomes; Rubus caesius $\mathrm{L}$. root; (2:3:3:1)

$4.21 \quad$ Vaccinium myrtillus L. leaves; Phaseolus vulgaris L. pericarp; Arctium lappa L. roots; Vaccinium vitis-idaea $\mathrm{L}$. leaves; $(2: 2: 1: 1)$

4.22 Cichorium intybus L. roots; Plantago major L. leaves; Arctium lappa L. roots; Equisetum arvense L. aerial part; (1:1: $1: 1)$

4.23 Phaseolus vulgaris L. pericarp; Vaccinium myrtillus L. leaves; Taraxacum campylodes G.E.Haglund. roots; Urtica dioica L. leaves; (1:1:1:1)

4.24 Juglans regia L. leaves; Vaccinium myrtillus L. leaves; Phaseolus vulgaris L. pericarp; Arctium spp. ${ }^{\text {b }}$ roots; (1:1: $1: 1)$

4.25 Vaccinium myrtillus L. fruits; Sambucus nigra L. flowers; Arctium lappa L. roots; Zea mays L. corn silk; (1:1:1:1)

4.26 Vaccinium myrtillus L. leaves; Phaseolus vulgaris L. pericarp; Polygonum aviculare L. aerial part; Arctostaphylos uva-ursi (L.) Spreng. leaves; $(1: 1: 1: 1)$

4.27 Galega officinalis L. aerial part; Vaccinium myrtillus L. leaves; Urtica dioica L. leaves; Taraxacum campylodes G.E.Haglund. roots; (1:1: 1:1)

4.28 Phaseolus vulgaris L. pericarp; Galega officinalis L. aerial part; Betula pendula Roth. leaves; Vaccinium myrtillus $\mathrm{L}$. leaves;(1:1:1:1)

4.29 Galega officinalis L. aerial part; Vaccinium vitis-idaea L. leaves; Frangula alnus Mill. bark; Betula pendula Roth. Leaves; (40:40:10:10)

4.30 Galega officinalis L. aerial part; Vaccinium myrtillus L. leaves; Sambucus nigra L. leaves; Viscum album L. aerial part; (7:7:4:2)

4.31 Vaccinium myrtillus L. leaves; Fragaria vesca L. leaves; Tilia cordata Mill. flowers; Verbascum densiflorum Bertol. flowers; (8:5:4:3)

4.32 Phaseolus vulgaris L. pericarp; Vaccinium myrtillus L. leaves; Laurus nobilis L. leaves; Morus alba L. leaves; $(1: 1: 1: 1)$
Herbal tea; 3 table spoons $100 \mathrm{ml} 7$ times a day

in $1,000 \mathrm{ml}$ of boiling

water

Infusion; 1 table spoon in $200 \mathrm{ml}$ of boiling water, boil $15 \mathrm{~min}$, maceration 30 min

Infusion; 1 table spoon in $300 \mathrm{ml}$ of boiling water, boil 3 min, maceration 10 min

Infusion; 1 table spoon in $200 \mathrm{ml}$ of boiling water maceration 20 min

Infusion; 1 table spoon in $200 \mathrm{ml}$ of cold water, maceration $1-2 \mathrm{~h}$ at room tempature, boil 5-7 min

Decoction

Infusion; $60 \mathrm{~g}$ in $300 \mathrm{ml}$ of boiling water

Infusion; 1 table spoon in $200 \mathrm{ml}$ of boiling water

Infusion; 2 table spoons in $400 \mathrm{ml}$ of boiling water, boil $10 \mathrm{~min}$, maceration 30-40 min Infusion; 3 table spoons in $600 \mathrm{ml}$ of boiling water, 15 min in boil water bath, maceration 30-40 min Infusion; 2 table spoons in $400 \mathrm{ml}$ of boiling water, $15 \mathrm{~min}$ in boil water bath, maceration 30-40 min Infusion; 2 table spoons in $400 \mathrm{ml}$ of boiling water, 15 min in boil water bath, maceration 30-40 min Infusion; 2-3 table spoons in $500 \mathrm{ml}$ of boiling water, 15 min in boil water bath, maceration 30-40 min before eating

$66 \mathrm{ml} 3$ times a day 20 min before eating after eating a day 30 min before $66 \mathrm{ml} 3$ times a day

$100 \mathrm{ml} 3$ times a day 30 min before eating

$130 \mathrm{ml} 3$ times a day 30 min before eating 30 min before eating
$200 \mathrm{ml} 3-4$ times a day

Indicatio

Diabetes

Diabetes

Rendiuk (2006)

Reference

dosage

$200 \mathrm{ml} 3-4$ times a day

Diabetes

$200 \mathrm{ml}$ 5-6 times a day

1-2 table spoons 3 times eating for 1-1.5 months

$200 \mathrm{ml} 3-4$ times a day

50-130 ml 2-3 times a

50-130 $\mathrm{ml} 2-3$ times a day 30 min before eating

$200 \mathrm{ml}$ 3-4 times a day
Diabetes

Diabetes

Diabetes

Diabetes

Diabetes

Diabetes

Sinyakov (1999)

Diabetes

Sinyakov (1999)

Diabetes

Sinyakov (1999)

Diabetes

Sinyakov (1999)

Kukes (1999) (2010)

Sinyakov (1999)
Nikolaychuk (1997),

Fedyukovich (1998), Onipko

(2002), Nikolaychuk and Zubitskaya (2003), Rendiuk (2006), Bogdanova and Bashkirova (2010), Pigulevskaya (2018)

Volynchenko (2003)

Volynchenko (2003)

Lager (1991), Lager (2002), Efimov and Shcherbak (1993), Nikolaychuk (1997), Fedyukovich (1998), Nikolaychuk and Zubitskaya (2003), Bogdanova and Bashkirova (2010) Lager (1991), Lager, (2002), Efimov and Shcherbak (1993), Bogdanova and Bashkirova

(Continued on following page) 
TABLE 1 | (Continued) The list of medical species used for the management of diabetes and related disorders in Russia.

Code $^{a} \quad$ Plant name, part used /(proportion) Method of preparation

\subsection{Vaccinium vitis-idaea L. leaves;} Taraxacum campylodes G.E.Haglund. leaves; Urtica dioica L. leaves; Galega officinalis L. aerial part; (1:1:1:1)

4.34 Vaccinium myrtillus L. leaves; Betula pendula Roth. leaves; Phaseolus vulgaris L pericarp; Urtica dioica L. leaves; (60:20:10:10)

4.35 Phaseolus vulgaris L. pericarp; Vaccinium myrtillus L. leaves; Rosa spp. ${ }^{6}$ fruits; Equisetum arvense $\mathrm{L}$. aerial part; $(4: 4: 4: 1)$

4.36 Vaccinium myrtillus L. leaves; Hypericum spp. eaerial part; Phaseolus vulgaris L. pericarp; Galega officinalis L. aerial part; $(4: 4: 4: 5)$

4.37 Avena sativa L. aerial part; Linum (usitatissimum L.) seeds; Phaseolus vulgaris L. pericarp; Vaccinium myrtillus L. leaves; $(1: 1: 1: 1)$

Infusion; 3 table spoons
$600 \mathrm{ml}$ of boiling water $500 \mathrm{ml}$ of boiling water,

boil 5-6 min

aceration $1-2 \mathrm{~h}$

$500 \mathrm{ml}$ of boiling water, boil $10 \mathrm{~min}$

maceration 1-2 $\mathrm{h}$

Infusion; $65 \mathrm{~g}$ in 1,000 ml

of water, boil 2 min

maceration 8-12 $\mathrm{h}$ in a

dark place

Infusion (herbal tea); 1

table spoon in $200 \mathrm{ml}$ of

boiling water
4.38 Arctostaphylos uva-ursi (L.) Spreng. leaves; Galega officinalis L. aerial part; Vaccinium myrtillus L. leaves; Valeriana officinalis L. roots; (1:1:1:1)
Infusion; 1 tea spoon in $200 \mathrm{ml}$ of boiling water

$50 \mathrm{ml}$ 6-8 times a day

Diabetes

Infusion; 2 table spoons in

$100 \mathrm{ml} 2$ times a day

before eating

$00 \mathrm{ml} 3$ times a day

before eating

Diabetes

Indication

Diabetes

Diabetes

$00 \mathrm{ml} 3$ times a day

$200 \mathrm{ml}$ 3-4 times a day Diabetes before eating
Infusion; 1 tea spoon in $200 \mathrm{ml}$ of boiling water alnus Mill. bark; Vaccinium myrtillus L. leaves; Galega officinalis L. aerial part; (1:1:4:4)

4.40 Vaccinium myrtillus L. leaves; Galega officinalis L. aerial part; Phaseolus vulgaris L. pericarp; Mentha $\times$ piperita L. leaves; (1:1:1:1)
Infusion; 2 table spoons in $500 \mathrm{ml}$ of boiling water
50-66 ml 3-4 times a day Diabetes $30 \mathrm{~min}$ before eating
$200 \mathrm{ml}$ 3-4 times a day Diabetes before eating
Reference

Sinyakov (1999)

Sinyakov (1999)

Brusenskaya and Kaz'min (2005)

Efimov and Shcherbak (1993), Brusenskaya and Kaz'min (2005), Davydovich et al. (2008), Bogdanova and Bashkirova (2010)

Makhlayuk (1991), Sinyakov (1992), Chirkov and Seryi (1993), Dmitriev et al. (1994), Nikultseva (1994), Tarasenko et al. (1998), Trofimenko and Mogilny, (1998), Sinyakov (1999), Podduev (2001), Volynchenko (2003), Popov (2004), Lavrenova and Lavrenov (2007), Davydovich et al. (2008), Pirogov (2008), Grechanyi (2013), Maznev (2014), Melik-Gusseinov and Rekkandt (2014)

Yordanov et al. (1972), Matkovskaya et al. (1988), Lager (1991), Sinyakov (1992), Sinyakov (1999), Chirkov and Seryi (1993), Nikolaychuk (1997), Tarasenko et al. (1998), Nikolaychuk and Zubitskaya (2003), Kiyanova (2005), Davydovich et al. (2008), Maznev (2014)

Yordanov et al. (1972), Lager (1991), Larger (2002), Chirkov and Seryi (1993), Efimov and Shcherbak (1993), Dontsov and Dontsov (2000), Bogdanova and Bashkirova (2010)

Yordanov et al. (1972), Matkovskaya et al. (1988), Lager (1991), Lager (2002); Sinyakov (1992), Sinyakov (1999), Chirkov and Seryi (1993), Efimov and Shcherbak (1993), Nikultseva (1994), Trofimenko and Mogilny (1998), Dontsov and Dontsov (2000), Blinov (2000), Dremova et al. (2003), Maznev (2005); Nazina (2006), Davydovich et al. (2008), Maznev (2014), Pigulevskaya (2018) (Continued on following page) 
TABLE 1 | (Continued) The list of medical species used for the management of diabetes and related disorders in Russia.

Code $^{a}$ Plant name, part used /(proportion) Method of preparation

4.41 Plantago major L. leaves; Taraxacum campylodes G.E.Haglund. leaves; Urtica dioica L. leaves; Vaccinium myrtillus L. leaves; $(1: 1: 1: 1)$
Infusion; 1 table spoon in $200 \mathrm{ml}$ of boiling water $20 \mathrm{~min}$ before eating

Indication

Reference

Recommended

$00 \mathrm{ml}$ 3-4 times a day Diabetes
4.42 Capsella bursa-pastoris (L.) Medik. aerial part; Equisetum arvense $\mathrm{L}$. aerial part; Polygonum aviculare $\mathrm{L}$ aerial part; Valeriana officinalis $\mathrm{L}$. roots and rhizomes; (1:1:1:1)

4.43 Galega officinalis L. aerial part; Juglans regia $\mathrm{L}$. leaves; Mentha $\times$ piperita $\mathrm{L}$. leaves; Polygonum aviculare L. aerial part ; $(1: 1: 1: 1)$
Infusion; 1 table spoon in $200 \mathrm{ml}$ of boiling water

Infusion; 1 table spoon in $200 \mathrm{ml}$ of boiling water
1 table spoon 3-4 times a Diabetes day 20-30 min before eating

$66 \mathrm{ml} 3$ times a day 15-20 min before eating
Diabetes
Sinyakov (1992), Sinyakov (1999), Efimov and Shcherbak (1993), Nikolaychuk (1997), Tarasenko et al. (1998), Fedyukovich (1998), Kukes (1999), Blinov (2000), Podduev (2001), Nikolaychuk and Zubitskaya (2003), Nazina (2006), Ryzhenko (2007), Davydovich et al. (2008), Bogdanova and Bashkirova (2010), Maznev (2014) Blinov (2000), Nazina (2006)
4.44 Cichorium intybus L. leaves; Fragaria vesca $L$ leaves; Polygonum aviculare L. aerial part; Taraxacum campylodes G.E.Haglund. leaves; (4:3:2:3)
Infusion; 1 table spoon in $66 \mathrm{ml}$ a day before eating Diabetes $200 \mathrm{ml}$ of boiling water
4.45 Mentha $\times$ piperita L. leaves; Ribes nigrum L. leaves; Rubus caesius L. leaves; Taraxacum campylodes G.E.Haglund. leaves; (1:3:2:4)

4.46 Helichrysum arenarium (L.) Moench flowers; Rosa majalis Herrm. fruits; Vaccinium myrtillus L. leaves; Zea mays L. corn silk; (1:2:5:2)

4.47 Alchemilla xanthochlora Rothm. aerial part; Juniperus communis L. fruits; Linum usitatissimum L. seeds: Vaccinium myrtillus L. leaves; (1:2:2:4)
Infusion; 1 table spoon in $200 \mathrm{ml}$ of boiling water

2-3 table spoons 3 times a day before eating

Infusion; 2 table spoons in $300 \mathrm{ml}$ of boiling water, maceration $12 \mathrm{~h}$ in thermos

Decoction; 1 table spoon in $200 \mathrm{ml}$ of water
$66 \mathrm{ml}$ 3-4 times a day $30 \mathrm{~min}$ before eating

Diabetes

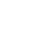
$200 \mathrm{ml}$ 2-3 times a day Diabetes

Diabetes

(1)


TABLE 1 | (Continued) The list of medical species used for the management of diabetes and related disorders in Russia.

Code $^{a}$ Plant name, part used /(proportion) Method of preparation

4.48 Galega officinalis L. aerial part; Mentha $\times$ piperita L. leaves; Phaseolus vulgaris L. pericarp; Zea mays L. corn silk; (1:1: 7:2)

4.49 Betula spp. 'Teaves; Frangula alnus Mill. bark; Vaccinium myrtillus L. leaves; Vaccinium vitis-idaea $L$ leaves; (1:1: 2:2)

4.50 Taraxacum campylodes G.E.Haglund. leaves; Urtica dioica L. leaves: Vaccinium myrtillus L. leaves; Vaccinium vitis-idaea L. leaves; (1:1: $1: 1)$

4.51 Avena sativa L. aerial part \& straw; Betula pendula Roth. leaves; Linum usitatissimum L. seeds; Vaccinium myrtillus L. leaves; (1:1:1:1)

4.52 Avena sativa L. aerial part \& straw; Equisetum arvense L. aerial part; Linum usitatissimum L. seeds; Oplopanax elatus (Nakai) Nakai roots and rhizomes; (2:2:2:1)

4.53 Arctostaphylos uva-ursi (L.) Spreng. leaves; Avena sativa L. aerial part \& straw; Linum usitatissimum L. seeds; Phaseolus vulgaris L. pericarp; (1:1: $1: 1)$

4.54 Juglans regia L. leaves; Mentha $\times$ piperita L leaves; Polygonatum odoratum (Mill.) Druce leaves;

Polygonum aviculare L. aerial part; (3:2: 2:3)

4.55 Aralia elata (Miq.) Seem roots; Galega officinalis L. aerial part; Rosa majalis Herrm. fruits; Vaccinium myrtillus $L$. fruits; (2:3:2:3)

4.56 Centaurium erythraea Rafn aerial part; Solanum tuberosum L. juice; Vaccinium myrtillus L. leaves; Viburnum opulus L. berries juice; (1:3:4:2)

4.57 Cichorium intybus L. roots; Rosa majalis Herrm. fruits; Schisandra chinensis (Turcz.) Baill. leaves; Taraxacum campylodes G.E.Haglund. roots; (3:4:1:3)

4.58 Betula pendula Roth. leaves; Ribes nigrum L. leaves; Rubus caesius L. leaves; Trifolium pratense L. leaves; (1: $1: 1: 1)$

4.59 Alchemilla xanthochlora Rothm. aerial part; Phaseolus vulgaris L. pericarp; Taraxacum campylodes G.E.Haglund. roots; Vaccinium myrtillus L. leaves; (1: $1: 1: 1)$

4.60 Achillea millefolium L. aerial part; Arctium spp. ${ }^{b}$ roots; Helichrysum arenarium (L.) Moench flowers; Hypericum spp. ${ }^{e}$ aerial part; (1:35:1:8)
Infusion; 3 table spoons in $400 \mathrm{ml}$ of boiling water

Decoction; Frangula bark cut, boil $20 \mathrm{~min}$ in boiling water + Infusion; in 300 m boiling water and boil 3 min

Infusion; 1 table spoon in $300 \mathrm{ml}$ of boiling water

Infusion; 3 table spoons in $600 \mathrm{ml}$ of boiling water, boil $10 \mathrm{~min}$, maceration 30-40 min Infusion; 1 table spoon in $200 \mathrm{ml}$ of boiling water, boil $15 \mathrm{~min}$, maceration $45 \mathrm{~min}$

Infusion; 1 table spoon in $200 \mathrm{ml}$ of boiling water, boil $10 \mathrm{~min}$

maceration $2 \mathrm{~h}$

Infusion; 2 table spoons in $500 \mathrm{ml}$ of boiling water, boil 2-3 min, maceration 30-40 min

Infusion or decoction; $10 \mathrm{~g}$ in $400 \mathrm{ml}$ of water

Infusion; $50 \mathrm{~g}$ in 1,000 ml of boiling water maceration 10-12 $\mathrm{h}$ at room temp.

Infusion; 1 tea spoon in boiling water

Infusion; 1 table spoon in $300 \mathrm{ml}$ of boiling water

Infusion; 1 table spoon in $200 \mathrm{ml}$ of water

Infusion; 2 table spoons in 300-400 ml of water

\section{Recommended} dosage

$100 \mathrm{ml} 3$ times a day Diabetes

Indication

Reference

$100 \mathrm{ml}$ 2-3 times a day before eating

Diabetes

$100 \mathrm{ml}$ 2-3 times a day Diabetes 20 min before eating

$50 \mathrm{ml}$ 6-8 times a day 20-30 min before eating

Diabetes

Podduev (2001)

$100 \mathrm{ml}$ a day

Diabetes

Podduev (2001)

$100 \mathrm{ml}$ 3-4 times a day

Diabetes 30 min before eating

66-100 $\mathrm{ml} 3$ times a day Low level

50-66 ml (warm) 3-4 times a day before eating

2 table spoons 4 times a day before eating and 30 min before sleeping for 30 days

$100 \mathrm{ml}$ with $1 / 2$ tea spoon of honey 3 times a day before eating

1 table spoon 3 times a day

Diabetes accompanied by impotence in men

Diabetes accompanied by gastritis

Diabetes accompanied by impotence in men

Diabetes accompanied by impotence in men

Brusenskaya and Kaz'min, (2005)

Brusenskaya and Kaz'min, (2005)

$100 \mathrm{ml}$ morning and evening
Diabetes accompanied by liver and gallbladder diseases
Brusenskaya and Kaz'min, (2005)

(Continued on following page) 
TABLE 1 | (Continued) The list of medical species used for the management of diabetes and related disorders in Russia.

Code $^{\mathrm{a}}$ Plant name, part used /(proportion) Method of preparation

4.61 Crataegus spp. ${ }^{f}$ lowers; Crataegus spp. fruits; Leonurus spp. deaves; Vaccinium myrtillus L. leaves; (1:1:1:4)

4.62 Asperula graveolens M.Bieb. ex Schult. \& Schult.f. aerial part; Fragaria vesca L. leaves; Melissa officinalis L. leaves; Thymus serpyllum L. aerial part; (1:2: $1: 1)$

4.63 Betula pendula Roth. leaves; Prunus spinosa L. flowers; Sambucus nigra L. flowers; Urtica dioica L. leaves; (1:1: $1: 1)$

4.64 Frangula alnus Mill. bark; Glycyrrhiza glabra L. roots; Viola tricolor L. aerial part; Juglans regia L. leaves; (1:1:4:4)

4.65 Betula pendula Roth. leaves; Melissa officinalis L. leaves; Salvia officinalis L. leaves; Urtica dioica L. leaves; (1:1:1:1)

4.66 Betula pendula Roth. leaves; Frangula alnus Mill. bark; Linum usitatissimum L. seeds; Urtica dioica L. leaves; (1:1:1:1)
Infusion; boiling of

Crataegus fruits $20 \mathrm{~min}$

than maceration of $30 \mathrm{~g}$ of

mixture in boiling water

8-12 h at room tempature

Infusion (herbal tea); 1

boiling water

Infusion (herbal tea); 1

table spoon in $200 \mathrm{ml}$ of

boiling water

Decoction; 1 table spoon in $600 \mathrm{ml}$ of water

Infusion (herbal tea); 1 table spoon in $200 \mathrm{ml}$ of boiling water

Decoction; 1 table spoon in $200 \mathrm{ml}$ of water

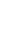

table spoon in $200 \mathrm{ml}$ of

\section{Recommended} dosage

$100 \mathrm{ml} 3$ times a day before eating

$100 \mathrm{ml} 4-5$ times a day

Metabolism improving

Chirkov and Seryi (1993)

Diabetes accompanied by Brusenskaya and Kaz'min, angina and shortness of (2005) breath

Indication

Reference

\section{5 plants}

5.1 Foeniculum vulgare Mill. fruits; Frangula alnus Mill. bark; Mentha $\times$ piperita L. leaves; Petroselinum crispum (Mill.) Fuss fruits; Taraxacum campylodes G.E.Haglund. roots; (1:3:1:1:1)

5.2 Achillea millefolium L. aerial part; Frangula alnus Mill. bark; Juniperus communis L. fruits; Levisticum officinale W.D.J.Koch roots; Phaeophyceae (Cystoseira barbata (Stackh.) C.Agardh) thallus; (10:3:1: 1:3)

5.3 Frangula alnus Mill. bark; Levisticum officinale W.D.J.Koch roots; Ononis spinosa L. roots; Phaeophyceae (Cystoseira barbata (Stackh.) C.Agardh) thallus; Taraxacum campylodes G.E.Haglund. roots; (5:1: $1: 2: 1)$

$5.4 \quad$ Matricaria chamomilla L. flowers; Foeniculum vulgare Mill. fruits; Mentha $\times$ piperita L. leaves; Sambucus nigra L. flowers; Tilia cordata Mill. flowers; (1:1: $1: 1: 1)$

\section{Infusion; 2 table spoons in} $500 \mathrm{ml}$ of boiling water

$500 \mathrm{ml}$ in the morning before eating

200-400 ml a day, before first eating

$400 \mathrm{ml}$ a day

$200 \mathrm{ml}$ in the morning and $200 \mathrm{ml}$ in the evening

$66 \mathrm{ml} 3$ times a day

66 mi times a day
Metabolism improving and diuretics

Exudative diathesis caused by metabolic disorder

Acne, in case of metabolic Chirkov and Seryi (1993) disorder

Skin rash, metabolic disorder
Chirkov and Seryi (1993)

Chirkov and Seryi (1993)

Chirkov and Seryi (1993)

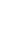


TABLE 1 | (Continued) The list of medical species used for the management of diabetes and related disorders in Russia.

Code $^{\mathrm{a}}$ Plant name, part used/(proportion) Method of preparation

Infusion; $10 \mathrm{~g}$ in $400 \mathrm{ml}$ of
water.

Arctostaphylos uva-ursi (L.) Spreng

leaves; Frangula alnus Mill. bark; Linum

usitatissimum L. seeds; Panax ginseng

C.A.Mey roots; Taraxacum

campylodes G.E.Haglund. roots; (3:4:

5:4:4)

5.6

Foeniculum vulgare Mill. fruits;

Hypericum perforatum L. aerial part;

Mentha $\times$ piperita L. leaves; Sambucus

nigra L. flowers Tilia cordata Mill.

flowers; (4:4:3:3:4)

5.7 Betula spp. ${ }^{\circ}$ leaves; Elymus repens(L.) Couldrhizomes; Frangula alnus Mill. bark; Melissa officinalis L. aerial part; Taraxacum campylodes G.E.Haglund. roots; (2:2:1:1:1)

5.8 Achillea millefolium L. aerial part; Carum carvi L. fruits; Frangula alnus Mill. bark; Viola tricolor L. aerial part; Zea mays L. corn silk; (1:1:6:1:1)

Humulus lupulus L. fruits; Panax ginseng C.A.Mey roots; Phaseolus vulgaris L. pericarp; Rosa majalis Herrm. fruits; Sorbus aucuparia L. fruits; (3:3:5:4:5)

5.10 Galega officinalis L. aerial part; Vaccinium myrtillus L. leaves; Phaseolus vulgaris L. pericarp; Taraxacum campylodes G.E.Haglund. roots; Urtica dioica L. leaves; (1:1:1: $1: 1)$
Infusion

Infusion; 2 table spoons in $500 \mathrm{ml}$ of boiling water, maceration $12 \mathrm{~h}$ in

thermos

Infusion; 4 table spoons in $800 \mathrm{ml}$ of boiling water

Infusion; $10 \mathrm{~g}$ in $400 \mathrm{ml}$ of water

Infusion; 1 table spoon in $200 \mathrm{ml}$ of boiling water
$200 \mathrm{ml} 3-4$ times a day before eating

2 table spoons 3 times a day 30 min before eating for 1-1.5 months

$100 \mathrm{ml} 3$ times a day

$400 \mathrm{ml} 2$ times a day

Obesity

66-100 ml 3 times a day

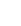

Recommended
Indication

Obesity

Obesity

ukes (1999)

Reference

Sokolov (2000) 
TABLE 1 | (Continued) The list of medical species used for the management of diabetes and related disorders in Russia.

Code $^{\mathrm{a}}$ Plant name, part used/(proportion) Method of preparation

5.14 Hypericum spp. ${ }^{e}$ flowers; Inula helenium L. roots; Sambucus nigra L. leaves; Taraxacum campylodes G.E.Haglund. leaves; Urtica dioica L. leaves; (2:1:2:2:1)

5.15 Arctium lappa L. roots; Galega officinalis L. aerial part; Oenanthe aquatica (L.) Poir. fruits; Polygonum aviculare L aerial part; Symphytum officinale L. root; (4:7:3:3:3)

5.16 Galega officinalis L. aerial part; Taraxacum campylodes G.E.Haglund. leaves; Urtica dioica L. leaves; Vaccinium myrtillus L. leaves; Vaccinium vitis-idaea L. leaves; (1:1:1: $1: 1)$
5.17 Galega officinalis L. aerial part; Phaseolus vulgaris L. pericarp; Taraxacum campylodes G.E.Haglund. roots; Urtica dioica L. leaves; Vaccinium myrtillus L. leaves; (1:1:1: $1: 1)$

5.18 Betula spp. 'leaves; Frangula alnus Mill. bark; Galega officinalis L. aerial part; Vaccinium myrtillus L. leaves; Vaccinium vitis-idaea L. leaves; (1:1:2: 2:2)

5.19 Cichorium intybus L. leaves; Galega officinalis L. aerial part; Juglans regia L. leaves; Taraxacum campylodes G.E.Haglund. leaves; Urtica dioica L. leaves; (1:1:1:1:1)
Infusion; 1 table spoon in $200 \mathrm{ml}$ of boiling water

$66 \mathrm{ml}$ 3-4 times a day before eating

Infusion; $7 \mathrm{~g}$ in $300 \mathrm{ml}$ of boiling water + Decoction Frangula bark cut in $300 \mathrm{ml}$ of boil warter, boil 20 min
Infusion; 1 table spoon in $400 \mathrm{ml}$ of boiling water

-3 table spoons 3 times a day 15-20 min before eating
5.20 Elymus repens (L.) Gould rhizomes; Sambucus nigra L. flowers; Tilia cordata Mill. flowers; Tussilago farfara L. leaves; Verbascum densiflorum Bertol. flowers; (1:1:1:1:1)

5.21 Althaea officinalis L. roots; Centaurium erythraea Rafn aerial part;Mentha $\times$ piperita L. aerial part; Prunusavium(L.) L. shoots; Zea mays L. corn silk; (1:1:5: $1: 1)$
Decoction; 5 table spoons $66 \mathrm{ml}$ 5-6 times a day Diabetes in $600 \mathrm{ml}$ of water

$90 \mathrm{~g}$ in $2000 \mathrm{ml}$ of boiling water; evaporate to residue of $1,000 \mathrm{ml}$

Diabetes
Diabetes

(a)

$150 \mathrm{ml}$ in the morning before eating, than 1 table spoon every $2 \mathrm{~h}$ during the day
Diabetes

Indication Diabetes

Diabetes

Diabetes $20 \mathrm{~min}$ before eating

$66 \mathrm{ml}$ before each eating Diabetes

Sinyakov (1992); Efimov and Shcherbak (1993); Nikultseva (1994); Nikolaychuk (1997); Tarasenko et al. (1998); Fedyukovich (1998); Blinov (2000); Podduev (2001); Nikolaychuk and Zubitskaya (2003); Nazina (2006); Davydovich et al. (2008); Bogdanova and Bashkirova (2010); Maznev (2014) Sinyakov (1992); Efimov and Shcherbak (1993); Nikultseva (1994); Nikolaychuk (1997); Fedyukovich (1998); Blinov (2000); Nikolaychuk and Zubitskaya (2003); Dremova et al. (2003); Nazina (2006);

Ryzhenko (2007);

Bogdanova and Bashkirova (2010) Chirkov and Seryi (1993) Osetrov (1993); Osetrov and Shreter (2001)

(Continued on following page) 
TABLE 1 | (Continued) The list of medical species used for the management of diabetes and related disorders in Russia.

Code $^{\mathrm{a}}$ Plant name, part used /(proportion) Method of preparation

5.22 Arctostaphylos uva-ursi (L.) Spreng. leaves; Mentha $\times$ piperita $L$. leaves; Ribes nigrum L. leaves; Rubus caesius L. leaves; Vaccinium myrtillus L. leaves; $(1: 1: 1: 1: 1)$

5.23 Arctium spp. ' roots; Juglans regia L. leaves; Phaseolus vulgaris L. pericarp Sambucus nigra L. flowers or roots; Vaccinium myrtillus L. leaves; (1:1:1: $1: 1)$

5.24 Equisetum arvense L. aerial part; Hypericum perforatum L. aerial part; Taraxacum campylodes G.E.Haglund. roots; Urtica dioica L. leaves;

Vaccinium myrtillus L. leaves; (1:1:1: $1: 1)$

5.25 Cichorium intybus L. roots; Crataegus spp. fruits; Elymus repens (L.) Gould rhizomes; Rosa spp. '9fruits; Vaccinium myrtillus L. fruits; (3:2:3:2:2)

5.26 Sambucus nigra L. flowers; Taraxacum campylodes G.E.Haglund. roots Urtica dioica L. leaves; Vaccinium myrtillus $\mathrm{L}$. fruits; Vaccinium myrtillus L. leaves; (3: 3:4:4:4)

5.27 Arctium lappa L. roots; Phaseolus vulgaris L. pericarp; Urtica dioica L. leaves; Vaccinium myrtillus L. leaves; Vaccinium vitis-idaea L. leaves; (1:1:1: $1: 1)$

5.28 Cichorium intybus L. roots; Hypericum perforatum L. aerial part; Mentha $\times$ piperita L. leaves; Taraxacum campylodes G.E.Haglund. roots; Vaccinium myrtillus L. leaves; (1:1:1: $1: 1)$

5.29 Cichorium intybus L. leaves; Galega officinalis L. aerial part; Juglans regia L. leaves; Taraxacum campylodes G.E.Haglund. leaves; Urtica dioica L. leaves; (1:1:1:1:1)

5.30 Galega officinalis L. aerial part; Phaseolus vulgaris L. pericarp; Taraxacum campylodes G.E.Haglund. roots; Urtica dioica L. leaves; Vaccinium myrtillus L. leaves; (1:1:1: $1: 1)$

5.31 Betula pendula Roth. leaves; Frangula alnus Mill. bark; Galega officinalis L. aerial part; Vaccinium myrtillus L. leaves; Vaccinium vitis-idaea $L$. leaves; $(3: 2: 5: 5: 5)$
Infusion; 2 table spoons in $500 \mathrm{ml}$ of boiling water, boil 2-3 min, maceration 30-40 min

Infusion; 2 table spoons in $500 \mathrm{ml}$ of boiling water, maceration $12 \mathrm{~h}$ in thermos

Decoction; Frangula bark boil for 20 min. Infusion; other part in $500 \mathrm{ml}$ of boiling water, boil for 3-4 min, maceration $30 \mathrm{~min}$. Mix with frangula decoction.
Infusion; $100 \mathrm{~g}$ in $400 \mathrm{ml}$ of boiling water, maceration $5 \mathrm{~h}$

Infusion; 1 table spoon in $200 \mathrm{ml}$ of boiling water, maceration $30 \mathrm{~min}$

Infusion; 1 table spoon in $200 \mathrm{ml}$ of boiling water, boil $10 \mathrm{~min}$, maceration at room tempature Infusion

Infusion; $20 \mathrm{~g}$ in $200 \mathrm{ml}$ of boiling water

Infusion; 1 table spoon in $300 \mathrm{ml}$ of boiling water

$66 \mathrm{ml} 3$ times a day

$100 \mathrm{ml} 4$ times a day $30 \mathrm{~min}$ before eating.

1-2 table spoon 3 times a day 30 min before eating for 1-1.5 months.

$66 \mathrm{ml} 3$ times a day
$50 \mathrm{ml} 3-4$ times a day 15-20 min before eating

$100 \mathrm{ml}$ (warm) 3 times a day 30 min before eating
Diabetes

Indication

66-100 $\mathrm{ml}$ 2-3 times a day 20-30 min before eating
Efimov and Shcherbak (1993); Nikolaychuk (1997);

Fedyukovich (1998); Smolianskil and Lifliandskii (2004);

Bogdanova and Bashkirova (2010); Melik-Gusseinov and Rekkandt (2014)

Smolianskii and Lifliandskii

(2004); Pigulevskaya (2018)
Diabetes

Diabetes

Lager (1991), Lager (2002)

Diabetes

Din

Diabetes

Diabetes

Diabetes

Diabetes

Sinyakov (1999)

Efimov and Shcherbak (1993); Nikolaychuk (1997); Fedyukovich (1998); Nikolaychuk and Zubitskaya (2003); Smolianskii and Lifliandskii (2004); Bogdanova and Bashkirova (2010) Efimov and Shcherbak (1993); Rendiuk (2006); Bogdanova and Bashkirova (2010)

Kukes (1999)

Lager (1991), Lager (2002); Efimov and Shcherbak (1993); Nikolaychuk (1997); Fedyukovich (1998); Nikolaychuk and Zubitskaya (2003); Bogdanova and Bashkirova (2010); Maznev (2014); Pigulevskaya (2018) Sinyakov (1999)

Diabetes Sinyakov (1999)

(Continued on following page) 
TABLE 1 | (Continued) The list of medical species used for the management of diabetes and related disorders in Russia.

Code $^{\mathrm{a}}$ Plant name, part used /(proportion) Method of preparation

5.32 Arctium lappa L. roots; Cichorium intybus L. roots; Linum usitatissimum L. seeds; Phaseolus vulgaris L. pericarp; Vaccinium myrtillus L. leaves; (2:2:2:7:7)

5.33 Alchemilla xanthochlora Rothm. roots and aerial part; Phaseolus vulgaris L. pericarp; Taraxacum campylodes G.E.Haglund. roots; Urtica dioica L. leaves; Vaccinium myrtillus L. leaves (1: $1: 1: 1: 1)$

5.34 Angelica archangelica L. aerial part; Betula spp. Leaves; Frangula alnus Mill. bark; Vaccinium myrtillus L. leaves; Vaccinium vitis-idaea $L$. leaves; (2:1:1 2:2)

5.35 Avena sativa L. aerial part; Fagopyrum esculentum Moench flowers; Linum usitatissimum L. seeds; Prunus laurocerasus L. leaves; Sambucus ebulus L. flowers; (3:2:2:3:2)

5.36 Acorus calamus L. root; Arctium spp. 'leaves; Matricaria chamomilla L. flowers; Frangula alnus Mill. bark; Vaccinium myrtillus L. leaves; (1:3:2:1:4)

5.37 Arctium lappa L. roots; Cichorium intybus L. roots; Rubus caesius L. root; Valeriana officinalis $\mathrm{L}$. roots and rhizomes; Vincetoxicum hirundinaria Medik. roots, rhizomes, leaves and seeds; (2:3:1:3:3)

5.38 Arctium lappa L. roots; Equisetum arvense L. aerial part; Gratiola officinalis L. aerial part; Orthosiphon aristatus (Blume) Miq. leaves; Phaseolus vulgaris L. pericarp; (2:3:2:1:4)

5.39 Asparagus officinalis L. rhizomes and aerial part; Centaurium erythraea Rafn aerial part; Fraxinus excelsior L. leaves Oplopanax elatus (Nakai) Nakai roots and rhizomes; Plantago major $\mathrm{L}$. leaves; (2:2:3:2:3)

5.40 Equisetum arvense L. aerial part; Gnaphalium uliginosum L. aerial part; Rosa majalis Herrm. fruits; Sambucus nigra L. flowers; Syringa vulgaris L. buds; (3:3:2:2:2)

5.41 Centaurium erythraea Rafn aerial part; Cichorium intybus L. roots; Hypericum

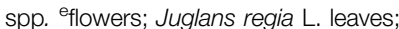
Plantago major L. leaves; (1:2:4:1:3)

5.42 Alnus spp. (A. incana (L.) Moench and A. glutinosa (L.) Gaertn.) fruits; Centaurium erythraea Rafn aerial part; Mentha $\times$ piperita L. leaves; Quercus

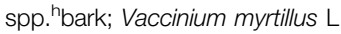
leaves; (2:1:1:4:4)

5.43 Betula spp. 'sap; Daucus sativusRoehl. juice; Leonurus spp deaves; Phaseolus vulgaris L. pericarp; Viburnum opulus L. berries juice; (20:2:1:4:2)

Infusion; 2-3 table spoons

in $500 \mathrm{ml}$ water,

maceration $12 \mathrm{~h}, 15 \mathrm{~min}$

in boil water bath,

maceration $1 \mathrm{~h}$

Infusion; 1 table spoon in $200 \mathrm{ml}$ of water

Decoction; Frangula bark cut boil 20 min + Infusion; other part in $300 \mathrm{ml}$ of boiling water, 3 min boil. Mix with frangula decoction. in $800 \mathrm{ml}$ of boiling water

Infusion; $55 \mathrm{~g}$ in 1,000 ml

of boiling water,

room tempature

in $1,000 \mathrm{ml}$ of boiling

water

Herbal tea; 3 table spoons in $1,000 \mathrm{ml}$ of boiling water

Herbal tea; 3 table spoons in $800 \mathrm{ml}$ of boiling water

Herbal tea; 3 table spoons

water

Decoction; Cichorium roots in $100 \mathrm{ml}$ of water Infusion; $45 \mathrm{~g}$ other part in $1,000 \mathrm{ml}$ of boil water, maceration $3-5 \mathrm{~h}$. Mix with cichorium decoction Infusion; $60 \mathrm{~g}$ in 1,000 ml of water, maceration 3-4 h at room tempature

Infusion; $40 \mathrm{~g}$ in 1,000 ml of boil water, maceration 3-5 h in a dark place
Herbal tea; 3 table spoons

$50 \mathrm{ml} 6$ times a day

$70 \mathrm{ml} 2-3$ times a day before eating

$200 \mathrm{ml}$ 3-4 times a day

Diabetes

Indication

Reference maceration $10-12 \mathrm{~h}$ at

Herbal tea; 3 table spoons $100 \mathrm{ml} 7$ times a day in $1,200 \mathrm{ml}$ of boiling
$50 \mathrm{ml} 6$ times a day

$70 \mathrm{ml} 6$ times a day

Diabetes accompanied by edema related to renal failure condition of the disease course

Diabetes accompanied by colitis and constipation

Brusenskaya and Kaz'min (2005)

Diabetes accompanied by metabolic polyarthritis,

Protasenya and Vasilenko rheumatoid arthritis

(1992)

Protasenya and Vasilenko (1992)

Diabetes accompanied by chronic gastritis with

Protasenya and Vasilenko (1992) reduced secretory function

$100 \mathrm{ml} 6$ times a day

Diabetes accompanied by hypertension and slight

Protasenya and Vasilenko edema of the lower extremities

$50 \mathrm{ml} 3$ times a day before eating

$50 \mathrm{ml}$ 3-4 times a day before eating, for 7-10 days

$100 \mathrm{ml} 4-6$ times a day before eating
Diabetes accompanied by angina and shortness of breath
Diabetes accompanied by colitis and constipation

Efimov and Shcherbak (1993); Brusenskaya and Kaz'min (2005); Davydovich et al. (2008); Bogdanova and Bashkirova (2010)

Diabetes with frequent diarrhea

Brusenskaya and Kaz'min (2005)

Brusenskaya and Kaz'min (2005)

(Continued on following page) 
TABLE 1 | (Continued) The list of medical species used for the management of diabetes and related disorders in Russia.

\begin{tabular}{|c|c|c|c|c|c|}
\hline Code $^{a}$ & Plant name, part used /(proportion) & Method of preparation & $\begin{array}{c}\text { Recommended } \\
\text { dosage }\end{array}$ & Indication & Reference \\
\hline 5.44 & $\begin{array}{l}\text { Polygonum aviculare L. aerial part Zea } \\
\text { mays L. corn silk; Hypericum spp. }{ }^{\text {e }} \\
\text { flowers; Viburnum opulus L. berries; } \\
\text { Arctostaphylos uva-ursi (L.) Spreng. } \\
\text { leaves; (1:2:2:1:2) }\end{array}$ & $\begin{array}{l}\text { Infusion; } 40 \mathrm{~g} \text { in 1,000 ml } \\
\text { of boil water, maceration } \\
3-5 \mathrm{~h} \text { in a dark place }\end{array}$ & $\begin{array}{l}100 \mathrm{ml} 3-4 \text { times a day } \\
\text { after eating }\end{array}$ & $\begin{array}{l}\text { Diabetes accompanied by } \\
\text { kidney and bladder } \\
\text { disease }\end{array}$ & $\begin{array}{l}\text { Brusenskaya and Kaz'min } \\
(2005)\end{array}$ \\
\hline 5.45 & $\begin{array}{l}\text { Anethum graveolens L. fruits; Mentha } \times \\
\text { piperita L. leaves; Crataegus sanguinea } \\
\text { Pall. flowers; Helichrysum arenarium } \\
\text { (L.) Moench. flowers; Matricaria } \\
\text { chamomilla L. flowers; (3:3:2:2:2) }\end{array}$ & $\begin{array}{l}\text { Infusion (herbal tea); } 1 \\
\text { table spoon in } 200 \mathrm{ml} \text { of } \\
\text { boiling water }\end{array}$ & $\begin{array}{l}100 \mathrm{ml} 3 \text { times a day } 1 \mathrm{~h} \\
\text { after eating }\end{array}$ & $\begin{array}{l}\text { Diabetes accompanied by } \\
\text { chronic pancreatitis }\end{array}$ & $\begin{array}{l}\text { Chirkov and Seryi (1993); Kukes } \\
\text { (1999); Bubenchikova et al. } \\
\text { (2003) }\end{array}$ \\
\hline 5.46 & $\begin{array}{l}\text { Taraxacum campylodes G.E. Haglund. } \\
\text { roots; Arctium lappa L. roots; Rubia } \\
\text { tinctorum L. roots; Saponaria officinalis } \\
\text { L. roots or Bidens tripartita L. aerial part; } \\
\text { Glycyrrhiza glabra L. roots; (3:3:6:6:2) }\end{array}$ & $\begin{array}{l}\text { Decoction; } 1 \text { table spoon } \\
\text { in } 200 \mathrm{ml} \text { of water }\end{array}$ & $\begin{array}{l}200-400 \mathrm{ml} \text { a day before } \\
\text { first eating }\end{array}$ & $\begin{array}{l}\text { Exudative diathesis } \\
\text { caused by metabolic } \\
\text { disorder }\end{array}$ & Chirkov and Seryi, (1993) \\
\hline 5.47 & $\begin{array}{l}\text { Humulus lupulus L. fruits; Menyanthes } \\
\text { trifoliata L. leaves; Gentiana lutea L. } \\
\text { root; Melissa officinalis L. aerial part; } \\
\text { Achillea millefolium L. aerial part; (2:3:2: } \\
4: 1)\end{array}$ & $\begin{array}{l}\text { Herbal tea; } 3 \text { table spoons } \\
\text { in 1,000 } \mathrm{ml} \text { of boiling } \\
\text { water }\end{array}$ & $100 \mathrm{ml} 6$ times a day & $\begin{array}{l}\text { Metabolic disorder with } \\
\text { multiple skin furuncles }\end{array}$ & $\begin{array}{l}\text { Protasenya and Vasilenko } \\
\text { (1992) }\end{array}$ \\
\hline
\end{tabular}

\section{6 plants}

6.1 Achillea millefolium L. aerial part; Carum carvi L. fruits; Frangula alnus Mill. bark; Prunus spinosa L. flowers; Viola tricolor $\mathrm{L}$. aerial part; Zea mays $\mathrm{L}$. corn silk; (1:1:6:1:1:1)

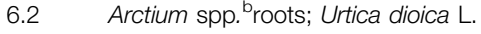
leaves; Avena sativa L. aerial part; Vaccinium myrtillus $L$. leaves; Fragaria vesca L. leaves; Rosa spp. 9fruits (3:4: 4:4:3:4)

6.3 Vaccinium myrtillus L. leaves; Rosa spp. 9fruits; Phaseolus vulgaris $L$. pericarp; Fragaria vesca L. leaves; Taraxacum campylodes G.E.Haglund. roots; Achillea millefolium $\mathrm{L}$. aerial part; (4:5:4:3:2:1)

6.4 Avena sativa L. aerial part; Cichorium intybus L. roots; Galega officinalis L. aerial part; Linum (usitatissimum L.) seeds; Phaseolus vulgaris L. pericarp; Vaccinium myrtillus $L$. leaves; (1:1:1:1: 1:1)

6.5 Urtica dioica L. leaves; Arctium lappa L. roots; Linum usitatissimum L. seeds; Juniperus communis $L$. fruits; Taraxacum campylodes G.E.Haglund. roots; Vaccinium myrtillus $L$. leaves; (2:2:1:1:1:3)

6.6 Linum usitatissimum L. seeds; Vaccinium vitis-idaea L. leaves; Inula helenium L. roots; Gnaphalium uliginosum $\mathrm{L}$ aerial part; Zea mays $\mathrm{L}$. corn silk; Matricaria chamomilla $L$. flowers; (4:4:3:3:3:3)

6.7 Mentha $\times$ piperita $L$ aerial part; Rosa spp. 9fruits; Sorbus aucuparia L. fruits; Taraxacum campylodes G.E.Haglund. roots; Urtica dioica L. leaves; Vaccinium myrtillus $L$. shoots; (1:1:1:1: 1:1) in $400 \mathrm{ml}$ of water

$400 \mathrm{ml} 2$ times a day

Obesity

Chirkov and Seryi (1993)
Herbal tea; 1 table spoon in $200 \mathrm{ml}$ of boiling water

Herbal tea; 1 table spoon in $200 \mathrm{ml}$ of boiling water

Infusion; $10 \mathrm{~g}$ in $300 \mathrm{ml}$ of boiling water, maceration $2 \mathrm{~h}$

Infusion; 3 table spoon in $600 \mathrm{ml}$ of boiling water

Infusion; 3 table spoons in $500 \mathrm{ml}$ of boiling water, maceration $12 \mathrm{~h}$ in

thermos

Infusion; $6 \mathrm{~g}$ in $350 \mathrm{ml}$ of boiling water, $10 \mathrm{~min}$ in boil water bath maceration $3 \mathrm{~h}$ in thermos
$100 \mathrm{ml} 2$ times a day before eating

$100 \mathrm{ml} 2$ times a day before eating

$100 \mathrm{ml} 3$ times a day before eating

$200 \mathrm{ml} 2-3$ times a day before eating

130-140 ml (warm) 3 times a day 20-30 min before eating

$100 \mathrm{ml} 3$ times a day before eating.
Diabetes prevention

nsulin-dependent

diabetes

Diabetes

\section{Diabetes}

Brusenskaya and Kaz'min (2005)

Brusenskaya and Kaz'min (2005); Maznev (2014)
(Continued on following page) 
TABLE 1 | (Continued) The list of medical species used for the management of diabetes and related disorders in Russia.

Code $^{a}$ Plant name, part used /(proportion) Method of preparation

\section{Arctium spp. ${ }^{\text {broots; }}$ Equisetum} arvense L. aerial part; Fragaria vesca L. leaves; Mentha $\times$ piperita $L$. aerial part; Vaccinium myrtillus L. shoots; Vaccinium vitis-idaea L. leaves; (1:1:1: $1: 1: 1)$ Betula spp. 'leaves; Foeniculum vulgare Mill. fruits; Mentha $\times$ piperita $\mathrm{L}$. aerial part; Petroselinum crispum (Mill.) Fuss aerial part; Ribes nigrum L. leaves; Rosa spp. 9fruits; (1:1:1:1:1:1)

6.10 Galega officinalis L. aerial part; Laurus nobilis L. leaves; Mentha $\times$ piperita $\mathrm{L}$. aerial part; Phaseolus vulgaris $\mathrm{L}$. pericarp; Sorbus aucuparia L. fruits; Vaccinium myrtillus L. leaves; (1:1:1:1:1:1)

6.11 Glycyrrhiza glabra L. roots; Hypericum spp. eaerial part; Juglans regia L. leaves; Phaseolus vulgaris L. pericarp; Syringa vulgaris L. buds; Vaccinium vitis-idaea L leaves; $(1: 1: 1: 1: 1: 1)$

6.12 Cichorium intybus L. roots; Elymus repens(L.) Couldroots; Fragaria vesca L. leaves; Rosa spp. Ileaves; Urtica dioica $\mathrm{L}$. leaves; Vaccinium myrtillus $\mathrm{L}$. leaves; (1:1:1:1:1:1)

6.13 Arctostaphylos uva-ursi (L.) Spreng. leaves; Fragaria vesca L. leaves; Vaccinium vitis-idaea L. leaves; Rosa spp. ${ }^{9}$ fruits; Vaccinium myrtillus $\mathrm{L}$. leaves; Urtica dioica L. leaves; (2:2:2:3: 4:1)

6.14 Ribes nigrum L. leaves; Hypericum

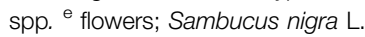
flowers; Urtica dioica L. leaves; Juglans regia $\mathrm{L}$. leaves; Fragaria vesca $\mathrm{L}$. leaves; (4:5:4:3:4:3)

6.15 Vaccinium myrtillus L. leaves; Urtica dioica L. leaves; Phaseolus vulgaris L. pericarp; Taraxacum campylodes G.E.Haglund. roots; Salvia officinalis L. leaves; Galega officinalis L. aerial part; (5:5:3:1:1:5)

6.16 Hypericum spp. ${ }^{e}$ aerial part; Achillea millefolium L. aerial part; Plantago major L. leaves; Arctium spp. ${ }^{b}$ roots; Centaurium erythraea Rafn aerial part; Matricaria chamomilla L. flowers; (6:2: 2:2:1:3)

6.17 Phaseolus vulgaris L. pericarp; Morus spp. Ileaves; Juglans regia L. leaves; Acorus calamus L. root; Frangula alnus Mill. bark; Ribes nigrum L leaves (20:5: 5:5:3:15)

6.18 Helichrysum (arenarium (L.) Moench. flowers; Hypericum spp. eaerial part; Polygonum aviculare L. aerial part; Rosa spp. 9fruits; Vaccinium myrtillus L. leaves; Zea mays L. corn silk; (2:2:3:2: 2:2) room tempature
Infusion; $8 \mathrm{~g}$ in $300 \mathrm{ml}$ of boiling water, $15 \mathrm{~min}$ in boil water bath,

maceration $1 \mathrm{~h}$ in thermos

Infusion; $8 \mathrm{~g}$ in $300 \mathrm{ml}$ of boiling water, $15 \mathrm{~min}$ in boil water bath, maceration $2 \mathrm{~h}$ in thermos

Infusion; $8 \mathrm{~g}$ in $400 \mathrm{ml}$ of boiling water, $15 \mathrm{~min}$ in

boil water bath, maceration $2 \mathrm{~h}$ in thermos

Infusion; 1 table spoon in $200 \mathrm{ml}$ of boiling water

Infusion; $70 \mathrm{~g}$ in 1,000 ml of water, maceration $3-5 \mathrm{~h}$ in a dark place

Infusion (herbal tea) 1 table spoon in $200 \mathrm{ml}$ (1 glass) of boiling water

Infusion; 1 table spoon in $500 \mathrm{ml}$ of boiling water maceration $12 \mathrm{~h}$ in thermos

Infusion; $80 \mathrm{~g}$ in 1,000 ml of boiling water, maceration 5-7 $\mathrm{h}$ at room temp.

Infusion; $53 \mathrm{~g}$ in 1,000 ml of boiling water, maceration $10-12 \mathrm{~h}$ at room temp.

Infusion; $65 \mathrm{~g}$ in 1,000 ml $50-70 \mathrm{ml}$ (warm) of boiling water maceration 10-12 $\mathrm{h}$ at before eating eating eating

3 table spoons eating
66-100 $\mathrm{ml}$ 3-4 times a day before eating

66-100 $\mathrm{ml}$ 3-4 times a day before eating

$100 \mathrm{ml}$ (warm) 3 times a day10 min before eating

$50 \mathrm{ml}$ several times a day Diabetes

Indication

Diabetes

$50-70 \mathrm{ml}($ warm
before eating

Vinogradova et al. (2001)

3-4 times a day after eating Diabetes

Brusenskaya and Kaz'min (2005)

100 ml, 2 times a day; Diabetes

$100 \mathrm{ml}$ (warm) 2-3 times a day 30 min before

$66 \mathrm{ml} 15-20 \mathrm{~min}$ before

Diabetes accompanied by colitis and constipation

Brusenskaya and Kaz'min (2005) 20-30 min before eating

Diabetes accompanied by colitis and constipation

Brusenskaya and Kaz'min, (2005) 3-4 times a day before
Sinyakov (1992), Sinyakov (1999); Chirkov and Seryi (1993)
Diabetes accompanied by liver and gallbladder diseases
Brusenskaya and Kaz'min (2005); Bogdanova and Bashkirova (2010)

(Continued on following page) 
TABLE 1 | (Continued) The list of medical species used for the management of diabetes and related disorders in Russia.

Code $^{a}$ Plant name, part used /(proportion) Method of preparation

6.19 Vaccinium myrtillus L. leaves; Helichrysum (arenarium (L.) Moench flowers; Zea mays L. corn silk; Polygonum aviculare L. aerial part; Hypericum spp. eaerial part; Phaseolus vulgaris L. pericarp; (4:1:2:1:2:2)

6.20 Achillea millefolium L. aerial part; Matricaria chamomilla L. flowers; Hypericum spp. eaerial part; Mentha $\times$ piperita L. leaves; Quercus spp. ${ }^{\mathrm{h}}$ bark; Tanacetum vulgare L. flowers; (30:8: 20:5:15:8)

6.21 Betula pendula Roth. leaves; Foeniculum vulgare Mill. fruits; Frangula alnus Mill. bark; Glycyrrhiza glabra L. roots; Sambucus nigra L. flowers; Viola tricolor L. aerial part; (1:1:1:1:1:1)

6.22 Arctium lappa L roots; Elymus repens (L.) Gould rhizomes; Foeniculum vulgare Mill. fruits; Frangula alnus Mill. bark; Glycyrrhiza glabra L. roots; Taraxacum campylodes G.E.Haglund. roots; $(1: 1: 1: 1: 1: 1)$

6.23 Arctostaphylos uva-ursi (L.) Spreng. leaves; Frangula alnus Mill. bark Herniaria (glabra) L. aerial part; Ononis spinosa L. roots; Saponaria officinalis L. roots; Solanum dulcamara L. aerial part; $(1: 1: 1: 1: 1: 1)$

6.24 "Normavit" Saccharina latissima (L.) C.E.Lane, C.Mayes, Druehl \& G.W.Saunders thallus; Rosa spp. Ifruits; Vaccinium vitis-idaea L. leaves; Leonurus spp daerial part; Bidens (tripartita L.) aerial part; Frangula alnus Mill. bark; (4:1:1:1:1:1)

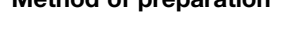

Infusion; $60 \mathrm{~g}$ in 1,000 ml of boiling water, maceration $10-12 \mathrm{~h}$ at room tempature

Infusion; $86 \mathrm{~g}$ in 1,000 m of boiling water, maceration 3-4 h at room tempature

Decoction; 1 table spoon in $200 \mathrm{ml}$ of boiling water

Decoction; 1 table spoon in $200 \mathrm{ml}$ of boiling water

Decoction; 1 table spoon in $200 \mathrm{ml}$ of boiling water

Decoction; $10 \mathrm{~g}$ in $130 \mathrm{ml}$ of boiling water

(20

50-100 ml 3 times a day Metabolism improving before eating for 20-30 days

Diabetes accompanied by liver and gallbladder 3-4 times a day before eating diseases morning before first

Reference

Indication

Brusenskaya and Kaz'min, (2005)

\section{$200 \mathrm{ml}$ (warm) in the} morning before first eating

$50 \mathrm{ml}$ 3-4 times a day before eating for

$7-10$ days

$66 \mathrm{ml} 3$ times a day

Skin rash, metabolic disorder

Metabolism improving Chirkov and Seryi, (1993)

200-400 $\mathrm{ml}$ in the eating

Metabolism improving and diuretics

Diabetes

Brusenskaya and Kaz'min, (2005)

Chirkov and Seryi, (1993)

\section{7 plants}

7.1 Betula spp. Meaves; Filipendula ulmaria(L.) Maxim. aerial part; Fragaria vesca L. leaves; Hypericum spp. eaerial part; Melissa officinalis L. aerial part;

Prunus spinosa L. flowers; Rosa spp. gruits; (1:1:1:1:1:1:1)

7.2 Achillea millefolium L. aerial part; Matricaria chamomilla L. flowers; Mentha $\times$ piperita L. leaves; Ribes nigrum L. leaves; Sorbus aucuparia L. fruits; Vaccinium myrtillus L. shoots; Vaccinium vitis-idaea L. leaves; (1:1:1: $1: 1: 1: 1)$

7.3 Tilia cordata Mill. flowers; Rosa majalis Herrm. fruits; Betula spp. 'leaves; Origanum vulgare $L$ aerial part; Hypericum perforatum L. aerial part; Calendula officinalis L. flowers; Ribes nigrum L. leaves; (3:3:1:1:1:2:2)
Infusion; $10 \mathrm{~g}$ in $300 \mathrm{ml}$ of $100 \mathrm{ml} \mathrm{3-4} \mathrm{times} \mathrm{a} \mathrm{day} \mathrm{Obesity}$

boiling water, boil $5 \mathrm{~min}$, maceration $2 \mathrm{~h}$ in thermos

$30 \mathrm{~min}$ before eating

ra
Samylina et al. (2010)
Infusion; $10 \mathrm{~g}$ in $300 \mathrm{ml}$ of boiling water, boil $5 \mathrm{~min}$, maceration $3 \mathrm{~h}$ in thermos

$100 \mathrm{ml} 3-4$ times a day 15 min before eating

Infusion; 2 table spoons in $2400 \mathrm{ml}$ of boiling water, maceration $8 \mathrm{~h}$ in thermos
$100 \mathrm{ml} 3$ times a day

Obesity
Vinogradova et al. (2001); Turishchev (2000), Turishchev (2005)

Vinogradova et al. (2001); Turishchev (2000), Turishchev (2005)

Kukes (1999); Bubenchikova et al. (2003)

(Continued on following page) 
TABLE 1 | (Continued) The list of medical species used for the management of diabetes and related disorders in Russia.

Code $^{\mathrm{a}}$ Plant name, part used /(proportion) Method of preparation

\subsection{Zea mays L. corn silk; Frangula alnus} Mill. bark; Cichorium intybus L. roots; Taraxacum campylodes G.E.Haglund. roots; Mentha $\times$ piperita $L$ leaves Petroselinum crispum (Mill.) Fuss fruits; Foeniculum vulgare Mill. fruits; (5:3:3:2: $1: 1: 1)$

"Arfazetin" Vaccinium myrtillus L. shoots; Phaseolus vulgaris L. pericarp; Aralia elata (Miq.) Seem roots; (or Oplopanax elatus (Nakai) Nakai roots and rhizomes); Rosa spp. Ifruits; Equisetum arvense L. aerial part; Hypericum perforatum L. aerial part; Matricaria chamomilla L. flowers; (4:4: 2:3:3:2:2)

Viburnum opulus L. berries; Vaccinium myrtillus L. fruits; Galega officinalis L. aerial part; Vaccinium vitis-idaea $L$ fresh berries (fruits); Hypericum spp. eflowers; Fragaria vesca L. leaves; Arctostaphylos uva-ursi (L.) Spreng. Leaves; (4:6:5:6:4:3:2)

7.7 Vaccinium myrtillus L. leaves; Urtica dioica L. leaves; Phaseolus vulgaris L. pericarp; Taraxacum campylodes G.E.Haglund. roots; Fragaria vesca L. leaves; Betula pendula Roth. leaves; Hypericum perforatum L. aerial part; (5: 2:5:2:2:2:2)

7.8 Avena sativa L. aerial part; Fragaria vesca L. aerial part; Linum usitatissimum L. seeds; Melissa officinalis L. aerial part; Rosa spp. Ifruits; Taraxacum campylodes G.E.Haglund. roots; Vaccinium vitisidaea L. leaves; (1:1:1:1:1:1:1)

7.9 Fragaria vesca L. aerial part; Galega officinalis L. aerial part; Helichrysum arenarium (L.) Moench flowers; Laurus nobilis L. leaves; Levisticum officinale W.D.J.Koch roots; Urtica dioica L. leaves; Vaccinium vitis-idaea $\mathrm{L}$ leaves; $(1: 1: 1: 1: 1: 1: 1)$

7.10 Equisetum arvense L. aerial part; Polygonum aviculare L. aerial part; Fragaria vesca L. leaves; Astragalus dasyanthus Pall. aerial part; Galega officinalis L. aerial part; Arnica montana L. flowers; Plantago major L. leaves; $(4: 4: 4: 3: 3: 1: 3)$

7.11 Equisetum arvense L. aerial part; Vaccinium myrtillus L. leaves; Juglans regia L. leaves; Phaseolus vulgaris L. pericarp; Fragaria vesca $L$ leaves; Matricaria chamomilla L. flowers; Cichorium intybus L leaves; (1:3:3:4:1: $1: 2)$
Infusion (herbal tea); 1 table spoon in $200 \mathrm{ml}$ of boiling water

Decoction; 1 table spoon in $600 \mathrm{ml}$ of water

Infusion; $10 \mathrm{~g}$ in $300 \mathrm{ml}$ of boiling water, $15 \mathrm{~min}$ in boil water bath,

maceration $1 \mathrm{~h}$ in thermos

Infusion; $10 \mathrm{~g}$ in $400 \mathrm{ml}$ of boiling water, $15 \mathrm{~min}$ in boil water bath, maceration $2 \mathrm{~h}$ in thermos

Infusion; 1 table spoons in $200 \mathrm{ml}$ of boiling water, boil 3-5 min, maceration 10-15 min at room tempature

Infusion; 2 table spoons in $400 \mathrm{ml}$ of boiling water, boil $15 \mathrm{~min}$
$100 \mathrm{ml} 2$ times a day before eating

before eating for 20-30 days 15 min before eating

70-100 ml 3-4 times a day before eating

2 table spoons 3-4 times a day 20-30 min before eating

$66 \mathrm{ml} 4$ times a day 20-30 min before eating
Lowering of blood glucose level, improving of glycogen-forming function of the liver, fortifying, antiinflammatory

Diabetes

Diabetes

Diabetes

Diabetes

Diabetes

Korsun et al. (2016)

Diabetes

Lavrenova and Lavrenov (2007)
Vinogradova et al. (2001)

Korotkova et al. (1988); Matkovskaya et al. (1988); Efimov and Shcherbak (1993); Nikultseva (1994); Sokolov (2000); Blinov (2000); Turishchev (2000), Turishchev (2005); Mashkovskii (2002); Dremova et al. (2003); Kiyanova (2005); Nazina (2006); Ryzhenko (2007); Davydovich et al. (2008); Vichkanova et al. (2009); Ruzhenkova (2014); Maznev (2014); Letova (2019); Register Russia (2021) Brusenskaya and Kaz'min, (2005)

Chirkov and Seryi (1993); Kukes (1999); Bubenchikova et al. (2003); Dremova et al. (2003)

Vinogradova et al. (2001) 
TABLE 1 | (Continued) The list of medical species used for the management of diabetes and related disorders in Russia.

\begin{tabular}{|c|c|c|c|c|c|}
\hline Code $^{a}$ & Plant name, part used /(proportion) & Method of preparation & $\begin{array}{l}\text { Recommended } \\
\text { dosage }\end{array}$ & Indication & Reference \\
\hline 7.12 & $\begin{array}{l}\text { Galega officinalis L. aerial part; } \\
\text { Phaseolus vulgaris L. pericarp; } \\
\text { Vaccinium myrtillus L. leaves; } \\
\text { Equisetum arvense L. aerial part; } \\
\text { Taraxacum campylodes G.E.Haglund. } \\
\text { roots; Urtica dioica L. leaves; } \\
\text { Gnaphalium uliginosum L. aerial part; } \\
\text { (2:2:2:1:1:1:1) }\end{array}$ & $\begin{array}{l}\text { Infusion; } 30 \mathrm{~g} \text { in } 400 \mathrm{ml} \text { of } \\
\text { boiling water, boil } 10 \mathrm{~min} \text {, } \\
\text { maceration } 1 \mathrm{~h}\end{array}$ & $\begin{array}{l}150 \mathrm{ml} 4 \text { times a day } \\
30 \mathrm{~min} \text { before eating }\end{array}$ & Diabetes & Lavrenova and Lavrenov (2007) \\
\hline 7.13 & $\begin{array}{l}\text { "Arfazetin E" Vaccinium myrtillus L. } \\
\text { shoots; Phaseolus vulgaris L. pericarp; } \\
\text { Eleutherococcus senticosus (Rupr. \& } \\
\text { Maxim.) Maxim. roots and rhizomes; } \\
\text { Rosa spp. }{ }^{9} \text { fruits; Equisetum arvense L. } \\
\text { aerial part; Hypericum perforatum L. } \\
\text { aerial part; Matricaria chamomilla L. } \\
\text { flowers (4:4:2:3:3:2:2) }\end{array}$ & $\begin{array}{l}\text { Infusion; } 10 \mathrm{~g} \text { in } 400 \mathrm{ml} \text { of } \\
\text { water }\end{array}$ & $\begin{array}{l}70-100 \mathrm{ml} 2-3 \text { times a } \\
\text { day before eating for } \\
20-30 \text { days }\end{array}$ & $\begin{array}{l}\text { Mild form of diabetes in } \\
\text { combination with diet and } \\
\text { exercise. Moderate } \\
\text { diabetes in combination } \\
\text { with oral hypoglycemic } \\
\text { drugs or insulin }\end{array}$ & Register Russia (2021) \\
\hline 7.14 & $\begin{array}{l}\text { Acorus calamus L. roots; Artemisia } \\
\text { absinthium L. aerial part; Bidens } \\
\text { tripartita L. aerial part; Mentha } \times \\
\text { piperita L. leaves; Origanum vulgare L. } \\
\text { aerial part; Pinus sylvestris L. buds; } \\
\text { Thymus serpyllum L. aerial part; (2:3:3: } \\
\text { 3:3:3:2) }\end{array}$ & $\begin{array}{l}\text { Herbal tea; } 6 \text { table spoons } \\
\text { in } 3,000 \mathrm{ml} \text { of boiling } \\
\text { water }\end{array}$ & For external use, baths & $\begin{array}{l}\text { Metabolic disorder with } \\
\text { skin furuncles }\end{array}$ & $\begin{array}{l}\text { Protasenya and Vasilenko } \\
\text { (1992) }\end{array}$ \\
\hline
\end{tabular}

\section{8 plants}

8.1 Arctostaphylos uva-ursi (L.) Spreng. leaves; Foeniculum vulgare Mill. fruits; Lavandula angustifolia Mill. leaves; Ononis spinosa L. roots; Persicaria hydropiper L. aerial part; Rheum palmatum L. roots; Rosa majalis Herrm. fruits; Senna alexandrina Mill. leaves; (2:2:2:2:2:1:1:1)

Agrimonia eupatoriaL.aerial part; Arctostaphylos uva-ursi (L.) leaves; Cetraria islandica L. thallus; Filipendula ulmaria(L.) Maxim.aerial part;Fumaria officinalis L.aerial part;Juglans regia L. leaves; Morusnigra L.leaves; Pinus silvestris L buds; (1:1:1:1:1:1:1:1) Achillea millefolium L. aerial part; Matricaria chamomilla L. flowers ; Elymus repens (L.) Gould rhizomes; Equisetum arvense L. aerial part; Fucus vesiculosus L. thallus; Hypericum perforatum L. aerial part; Melissa officinalis L. aerial part; Taraxacum campylodes G.E.Haglund. roots; (1:1: $1: 1: 2: 1: 1: 1)$

8.4 Achillea millefolium L., aerial part; Anethum graveolens L. fruits; Frangula alnus Mill. bark; Helichrysum arenarium (L.) Moench flowers; Orthosiphon aristatus (Blume) Miq. shoots; Rosa spp. 9fruits; Taraxacum campylodes G.E.Haglund. roots; Zea mays L. corn silk; $(1: 1: 1: 1: 1: 1: 1: 1)$

8.5 Tilia cordata Mill. flowers; Rosa spp. Ifruits; Betula spp. 'leaves; Origanum vulgare L. aerial part; Hypericum spp. eaerial part; Calendula officinalis L. flowers; Ribes nigrum L. leaves; Gnaphalium uliginosum L. aerial part; (3:3:1:1:1:1:2:2)
Decoction; 2 table spoons in $500 \mathrm{ml}$ of water

$100 \mathrm{ml} 4$ times a day

Obesity before eating

Infusion; 2 table spoons in $500 \mathrm{ml}$ of boiling water

$50 \mathrm{ml} 3$ times a day before eating

Obesity

Osetrov and Shreter (2001); Korsun and Korsun (2010)
Infusion; 1 tea spoon in $200 \mathrm{ml}$ of boiling water

Infusion; $15 \mathrm{~g}$ in $500 \mathrm{ml}$ of cold water, maceration $4 \mathrm{~h}$ at room temp., boil 3 min, maceration $1 \mathrm{~h}$ in thermos

Infusion; $10 \mathrm{~g}$ in $500 \mathrm{ml}$ of boiling water, maceration $6-8 \mathrm{~h}$ in thermos
$100 \mathrm{ml} 4-5$ times a day Obesity before eating

$125 \mathrm{ml} 3-4$ times a day before eating
Obesity and diabetes mellitus
Vinogradova et al. (2001); Turishchev (2000), Turishchev (2005) 
TABLE 1 | (Continued) The list of medical species used for the management of diabetes and related disorders in Russia. Code $^{\mathrm{a}}$ Plant name, part used /(proportion) Method of preparation Arctium spp. 'Pleaves; Avena sativa L. aerial part; Juniperus communis L. fruits; Linum usitatissimum L. seeds; Phaseolus vulgaris L. pericarp; Taraxacum campylodes G.E.Haglund. roots; Urtica dioica L. leaves; Vaccinium myrtillus L. leaves; (1:1:1:1: $1: 1: 1: 1)$

8.7 Equisetum arvense L. aerial part; Oplopanaxelatus(Nakai) Nakairoots and rhizomes; Taraxacum campylodes G.E.Haglund. roots; Rosa spp. ${ }^{9}$ fruits; Cichorium intybus L. roots; Linum usitatissimum L. seeds; Hypericum spp. ${ }^{e}$ aerial part; Tilia cordata Mill. flowers; (4:1:1:2:3:1:2:1)

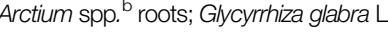
roots; Juglans regia L. leaves; Linum usitatissimum L. seeds; Rosa spp.' '9ruits; Sambucus nigra L. root; Vaccinium myrtillus L. shoots ; Viburnum opulus L. shoots; (1:1:1:1:1:1:1:1)

8.9 Matricaria chamomilla L. flowers; Viola tricolor L. aerial part; Equisetum arvense L. aerial part; Achillea millefolium L. aerial part;

Calendula officinalis L. flowers; Quercus spp. 'bark; Gnaphalium uliginosum $\mathrm{L}$ aerial part; Melilotus officinalis (L.) Pall.aerial part; (2:1:1:2:1: $2: 1: 1)$

8.10 Taraxacum campylodes G.E.Haglund. leaves; Vaccinium vitis-idaea L. leaves; Galega officinalis L. aerial part; Polygonum aviculare L. aerial part; Ribes nigrum L. leaves; Phaseolus vulgaris L. pericarp; Cichorium intybus L. leaves; Rosa spp. ${ }^{9}$ fruits; (1:4:4:1:2: 3:2:1)
Infusion; $8 \mathrm{~g}$ in $500 \mathrm{ml}$ of 1 table spoon 6 times boiling water, maceration a day $6-8 \mathrm{~h}$ in thermos

Infusion (herbal tea); 1 table spoon in $200 \mathrm{ml}$ of boiling water

Infusion; $10 \mathrm{~g}$ in $400 \mathrm{ml}$ of boiling water, $15 \mathrm{~min}$ in boil water bath, maceration $2 \mathrm{~h}$ in thermos

Infusion; 1 tea spoon in $200 \mathrm{ml}$ of boiling water, $15 \mathrm{~min}$ in boil water bath, maceration $45 \mathrm{~min}$ in thermos

Infusion; 2 table spoons in $400 \mathrm{ml}$ of boiling water, boil $8 \mathrm{~min}$, maceration $2 \mathrm{~h}$
$100 \mathrm{ml} 2$ times a day Diabetes before eating

70-100 ml 3-4 times a day before eating

$100 \mathrm{ml} 2$ times a day after Diabetes eating

$100 \mathrm{ml} 4$ times a day 20-30 min before eating
Lowering of blood glucose Podduev (2001) level

Indication

Reference 
TABLE 1 | (Continued) The list of medical species used for the management of diabetes and related disorders in Russia.

Code $^{a}$ Plant name, part used /(proportion) Method of preparation Matricaria chamomilla L. flowers; Equisetum arvense L. aerial part; Hypericum perforatum $L$ aerial part; Inula helenium L. roots; Mentha $\times$ piperita L leaves;

Oplopanaxelatus(Nakai) Nakairoots and rhizomes;Rosa majalis Herrm. fruits; Vaccinium myrtillus L. leaves; $(1: 1: 1: 1: 1: 1: 1: 2: 1)$

Bidens tripartita L. aerial part; Matricaria chamomilla L. flowers; Equisetum arvense L. aerial part; Fragaria vesca L roots; Hypericum perforatum L. aerial part; Inula helenium L. roots; Mentha $\times$ piperita L. leaves; Rosa majalis Herrm. fruits; Vaccinium myrtillus L. leaves; (1:1:1:4: $1: 1: 1: 1: 2)$

9.5 Crataegus sanguinea Pall. fruits; Rosa majalis Herrm. fruits; Urtica dioica L. leaves; Leonurus quinquelobatus Gilib. aerial part; Linum usitatissimum L. seeds; Mentha $\times$ piperita L. leaves; Asparagus officinalis L. rhizomes and aerial part; Thymus serpyllum L. aerial part; Vaccinium myrtillus L. leaves; (3:3: 3:5:2:1:4:4:7)

9.6 Carex arenaria L. rhizomes; CyanussegetumHillaerial part; Galega officinalis L. aerial part; Phaseolus vulgaris L. pericarp; Pimpinella saxifragaL. roots;Salvia officinalis $L$. leaves; Sambucus nigra L. flowers; Taraxacum campylodes G.E.Haglund. roots; Vaccinium myrtillus $L$. leaves;

$(1: 1: 1: 1: 1: 1: 1: 1: 2)$

9.7 Arctium lappa L. roots; Capsella bursapastoris (L.) Medik. aerial part; Hypericum perforatum L. aerial part; Juglans regia L. leaves; Mentha $\times$ piperita L. leaves; Rosa majalis Herrm. fruits; Vaccinium myrtillus L. leaves; Vaccinium vitis-idaea $L$. leaves; Zea mays L. corn silk; (6:3:3:4:1:4:4: 5:5)

9.8 Crataegus spp. fruits; Fragaria vesca L leaves; Hypericum spp. ${ }^{\text {eaerial part; }}$ Linum usitatissimum L. seeds; Phaseolus vulgaris L. pericarp; Plantago major L. leaves; Ribes nigrum L. leaves; Rosa spp. fruits; Vaccinium myrtillus L. leaves; (1:1:1:1:1:1:1:1:2)
Infusion; 1 table spoon in $200 \mathrm{ml}$ of boiling water

Infusion; 2-3 table spoons in $500 \mathrm{ml}$ of boiling water, maceration $12 \mathrm{~h}$ in thermos $500 \mathrm{ml}$ of boiling water, 15 min in boil water bath, maceration 30-40 min
Infusion; 2 table spoons in
Infusion; 2 table spoons in $500 \mathrm{ml}$ of boiling water, maceration $12 \mathrm{~h}$ in thermos Infusion; 3 table spoons in $500 \mathrm{ml}$ of boiling water, maceration $12 \mathrm{~h}$ in thermos
$66 \mathrm{ml} 3$ times a day before eating

$66 \mathrm{ml}$ (warm) 3 times a day 20-30 min before eating

$66 \mathrm{ml} 3$ times a day 30 min before eating
Diabetes

Diabetes Lowering of blood glucose level

Diabetes

Indication

Reference
$100 \mathrm{ml} 3-4$ times a day $30 \mathrm{~min}$ before eating

Diabetes Sinyakov (1999)

$140 \mathrm{ml}$ (warm) 3 times a

Sokolov and Zamotaiev (1984); Lisitsyn and Molodozhnikova (1989); Lager (1991), Lager (2002); Efimov and Shcherbak (1993); Nikultseva (1994); Tarasenko et al. (1998); Sinyakov (1999); Sokolov (2000); Dontsov and Dontsov (2000); Blinov (2000); Sokolov (2000); Dremova et al. (2003); Smolianskii and Lifliandskii (2004); Davydovich et al. (2008); Bogdanova and Bashkirova (2010); Ruzhenkova (2014); Maznev (2014)

Podduev (2001)
Ladynina and Morozova (1987), Ladynina and Morozova (1990); Blinov (2000); Sinyakov (1999); Nazina (2006); Davydovich et al. (2008); Bogdanova and Bashkirova (2010)

Sinyakov (1999) day 30 min before eating 
TABLE 1 | (Continued) The list of medical species used for the management of diabetes and related disorders in Russia.

Code $^{a}$ Plant name, part used /(proportion) Method of preparation
Recommended dosage
Reference

Indication

\section{0 plants}

10.1 Anethum graveolens L. fruits; Matricaria chamomilla L. flowers; Frangula alnus Mill. bark; Hypericum

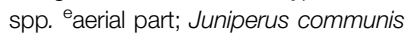
L. fruits; Prunus spinosa L. flowers; Rosa spp.9'9ruits; Taraxacum campylodes G.E.Haglund. roots; Urtica dioica L. leaves; Zea mays L. corn silk; (1:1:1:1:1:1:1:1:1:1)

10.2 Betula spp. 'leaves; Cichorium intybus L. roots; Frangula alnus Mill. bark; Glycyrrhiza glabra L. roots; Mentha $\times$ piperita L aerial part; Morus spp. ${ }^{i}$ leaves; Orthosiphon aristatus (Blume) Miq. shoots; Petroselinum crispum (Mill.) Fuss roots; Taraxacum campylodes G.E.Haglund. roots; Zea mays L. corn silk; (1:1:1:1:1:1:1:1:1:1)

10.3 Vaccinium vitis-idaea L. leaves; Zea mays L. corn silk; Syringa vulgaris L. buds; Arctium lappa L. roots; Mentha $\times$ piperita L. leaves; Juglans regia $L$ leaves; Hypericum perforatum L aerial part; Gnaphalium uliginosum L. aerial part; Vaccinium myrtillus L. leaves; Rosa majalis Herrm. fruits; (4:4:2:5:2:3: 2:2:3:1)

10.4 Achillea millefolium L. aerial part;

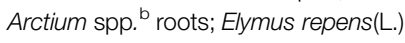
Gouldrhizomes; Fragaria vesca L. aerial part; Galega officinalis L. aerial part; Laurus nobilis L. leaves; Phaeophyceae (Cystoseira barbata (Stackh.) C.Agardh) thallus; Rosa spp. ${ }^{9}$ fruits; Trifolium pratense L. flowers; Vaccinium myrtillus L. leaves; (1:1:1:1:1:1:1:1:1:1)

10.5 Arctium lappa L. roots; Artemisia absinthium L. aerial part; Bidens tripartita L. aerial part;

Calendula officinalis L. flowers; Matricaria chamomilla L. flowers; Equisetum arvense L. aerial part; Gnaphalium uliginosum L. aerial part; Hypericum perforatum L. aerial part; Inula helenium L. roots; Salvia officinalis L. aerial part; $(1: 1: 1: 1: 1: 1: 1: 1: 1: 1)$
Infusion; $10 \mathrm{~g}$ in $300 \mathrm{ml}$ of boiling water, maceration $3 \mathrm{~h}$ in thermos

Infusion; $15 \mathrm{~g}$ in $500 \mathrm{ml}$ of boiling water, $15 \mathrm{~min}$ in boil water bath, maceration $2 \mathrm{~h}$ in thermos

Infusion; 2-3 table spoons in $500 \mathrm{ml}$ of boiling water, maceration $12 \mathrm{~h}$ in thermos

Infusion; $12 \mathrm{~g}$ in $350 \mathrm{ml}$ of boiling water, $10 \mathrm{~min}$ in boil water bath, thermos.

Decoction; 1-2 table spoons in $200 \mathrm{ml}$ of water maceration $2 \mathrm{~h}$ in
$100 \mathrm{ml} 3-4$ times a day

Obesity 15 min before eating

times a day 20 min before eating

Obesity

Vinogradova et al. (2001)
$66 \mathrm{ml}$ (warm) 3 times a Diabetes day,20-30 min before eating

$100 \mathrm{ml}$ (warm) 3 times a day10 min before eating
Diabetes

Vinogradova et al. (2001)
70-100 ml 3 times a day 30 min before eating
Diab

Diabetes accompanied by chronic pancreatitis
Chirkov and Seryi, (1993)
Vinogradova et al. (2001)
Ladynina and Morozova (1987), Ladynina and Morozova (1990); Blinov, 2000; Ladynina (2005); Nazina, (2006)

\section{1 plants}

11.1 Achillea millefolium L. aerial part; Arctium spp. 'roots; Cichorium intybus L. roots; Elymus repens(L.) Gouldroot; Inula helenium L. roots; Phaseolus vulgaris L. pericarp; Polygonum aviculare L. aerial part; Ribes nigrum L. leaves; Taraxacum campylodes G.E.Haglund. roots; Tilia cordata Mill. flowers; Vaccinium myrtillus L. leaves; $(1: 2: 1: 2: 1: 2: 2: 1: 2: 2: 2)$

Decoction; $10 \mathrm{~g}$ in $500 \mathrm{ml}$ of water, $2 \mathrm{~h}$ in boil water bath
00-150 ml 3-4 times a Insulin-dependent diabetes
Kolesova et al. (1998)

day before eating diabetes


TABLE 1 | (Continued) The list of medical species used for the management of diabetes and related disorders in Russia.

\begin{tabular}{|c|c|c|c|c|c|}
\hline Code $^{a}$ & Plant name, part used /(proportion) & Method of preparation & $\begin{array}{c}\text { Recommended } \\
\text { dosage }\end{array}$ & Indication & Reference \\
\hline 11.2 & $\begin{array}{l}\text { Betula pubescens Ehrh. leaves; } \\
\text { Crataegus sanguinea Pall. fruits; } \\
\text { Orthosiphon aristatus (Blume) Miq. } \\
\text { leaves; Rosa majalis Herrm. fruits; } \\
\text { Mentha × piperita L. leaves; Veronica } \\
\text { officinalis L. aerial part; Centaurium } \\
\text { erythraea Rafn aerial part; Arctium }\end{array}$ & $\begin{array}{l}\text { Infusion; } 2-3 \text { table spoons } \\
\text { in } 500 \mathrm{ml} \text { of boiling water, } \\
\text { maceration } 12 \mathrm{~h} \text { in } \\
\text { thermos }\end{array}$ & $\begin{array}{l}66 \mathrm{ml} \text { (warm) } 3 \text { times a } \\
\text { day 20-30 min before } \\
\text { eating }\end{array}$ & Diabetes & $\begin{array}{l}\text { Ladynina and Morozova (1987), } \\
\text { Ladynina and Morozova (1990); } \\
\text { Blinov (2000); Dremova et al. } \\
\text { (2003); Ladynina (2005); Nazina } \\
\text { (2006); Bogdanova and } \\
\text { Bashkirova (2010); Maznev } \\
\text { (2014) }\end{array}$ \\
\hline
\end{tabular}$$
\text { lappa L. roots; Leonurus }
$$

quinquelobatus Gilib. aerial part; Glycyrrhiza glabra L. roots; Cichorium intybus L. roots $(2: 3: 2: 2: 2: 1: 5: 5: 3: 2: 4)$

11.3 Vaccinium myrtillus L. leaves; Phaseolus vulgaris L. pericarp; Crataegus sanguinea Pall. Fruits; Hypericum perforatum L. aerial part; Plantago major L. leaves; Ribes nigrum L. leaves; Rosa majalis Herrm. fruits; Linum usitatissimum L. seeds; Mentha $\times$ piperita $L$ leaves; Fragaria vesca $L$ leaves Sambucus nigra L. flowers; (4:3:2:2:2:2:1:1:1:1:1)

11.4 Artemisia absinthium L. aerial part; Avena sativa L. aerial part; Urtica dioica L. leaves; Arctium spp. broots; Inula helenium L. roots; Alchemilla xanthochlora Rothm. aerial part; Taraxacum campylodes G.E.Haglund. leaves; Vaccinium myrtillus L. leaves; Gnaphalium uliginosum $L$ aerial part; Sambucus nigra L. flowers; Linum

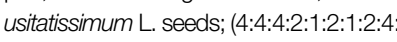
4:2)

11.5 Alchemilla xanthochlora Rothm. aerial part; Centaurium erythraea Rafn aerial part; Elymus repens(L.) Couldrhizomes; Gnaphalium uliginosum L aerial part; Juniperus communis L. fruits; Mentha $\times$ piperita $L$ aerial part; Rumex confertus Willd. roots; Sorbus aucuparia L. fruits; Syringa vulgaris L. buds; Taraxacum campylodes G.E.Haglund. roots; Trifolium pratense L. flowers; (1:1:1:1:1:1 1:1:1:1:1)

11.6 Arctium spp. ${ }^{\mathrm{b}}$ roots; Avena sativa L. aerial part; Betula spp. 'leaves; Galega officinalis L. aerial part; Glycyrrhiza glabra L. roots; Hypericum spp. eaerial part; Juglans regia L. leaves; Juniperus communis L. fruits; Laurus nobilis L. leaves; Vaccinium myrtillus L. leaves ; Vaccinium vitis-idaea $L$. leaves; $(1: 1: 1$ : $1: 1: 1: 1: 1: 1: 1: 1)$

11.7 Betula spp. 'leaves; Cyanus segetum Hill flowers; Foeniculum vulgare Mill. fruits; Laurus nobilis L. leaves; Linum usitatissimum L. seeds; Ononis spinosa L. roots; Petroselinum crispum (Mill.) Fuss roots; Phaseolus vulgaris L. pericarp; Taraxacum campylodes G.E.Haglund. roots; Urtica dioica L. roots; Viburnum opulus L. flowers; (1:1: 1:1:1:1:1:1:1:1:1)

Infusion; 3 table spoons in $500 \mathrm{ml}$ of boiling water. maceration $12 \mathrm{~h}$ in thermos

Infusion; 2 table spoons in $500 \mathrm{ml}$ of boiling water, maceration $12 \mathrm{~h}$ at room temp.

Infusion; $12 \mathrm{~g}$ in $350 \mathrm{ml}$ of boiling water, $10 \mathrm{~min}$ in boil water bath, maceration $2 \mathrm{~h}$ in thermos

Infusion; $10 \mathrm{~g}$ in $400 \mathrm{ml}$ of cold water, maceration $4 \mathrm{~h}$, boil $3 \mathrm{~min}$, maceration $2 \mathrm{~h}$ in thermos Infusion; $12 \mathrm{~g}$ in $400 \mathrm{ml}$ of boiling water, $15 \mathrm{~min}$ in boil water bath, maceration $1.5 \mathrm{~h}$ in thermos
130-140 ml (warm) 3 times a day 20-30 min before eating

$100 \mathrm{ml} 3$ times a day 15 min before eating.

$100 \mathrm{ml}$ (warm) 3 times a day 10 min before eating
Diabetes

Diabetes

Diabetes

Diabetes before eating

70-100 $\mathrm{ml} 3-4$ times a day 10 min before eating
Diabetes

Vinogradova et al. (2001)
Sinyakov (1992); Sinyakov (1999); Tarasenko et al. (1998); Davydovich et al. (2008)

Rendiuk (2006)

Vinogradova et al. (2001) 
TABLE 1 | (Continued) The list of medical species used for the management of diabetes and related disorders in Russia.

\begin{tabular}{|c|c|c|c|c|c|}
\hline Code $^{a}$ & Plant name, part used /(proportion) & Method of preparation & $\begin{array}{l}\text { Recommended } \\
\text { dosage }\end{array}$ & Indication & Reference \\
\hline 11.8 & $\begin{array}{l}\text { Arctium lappa L. roots; Centaurium } \\
\text { erythraea Rafn aerial part; Cichorium } \\
\text { intybus L. roots; Crataegus sanguinea } \\
\text { Pall. fruits; Rosa majalis Herrm. fruits; } \\
\text { Glycyrrhiza glabra L. roots; Leonurus } \\
\text { quinquelobatus Gilib. aerial part; } \\
\text { Orthosiphon aristatus (Blume) Miq. } \\
\text { leaves; Mentha × piperita L. leaves; } \\
\text { Betula nigra L. leaves; Veronica officinalis } \\
\text { L. aerial part; (3:3:2:2:2:2:2:1:1:1:1) }\end{array}$ & $\begin{array}{l}\text { Infusion; } 2-3 \text { table spoons } \\
\text { in } 500 \mathrm{ml} \text { of boiling water, } \\
\text { maceration } 12 \mathrm{~h} \text { in } \\
\text { thermos }\end{array}$ & $\begin{array}{l}130 \mathrm{ml} \text { (warm) } 3 \text { times a } \\
\text { day } 30 \text { min before eating }\end{array}$ & Diabetes & Sinyakov (1999) \\
\hline
\end{tabular}

\section{2 plants}

12.1 Crataegus sanguinea Pall. fruits; Mentha $\times$ piperita $L$. leaves; Rosa majalis Herrm. fruits; Sambucus nigra L. flowers; Hypericum perforatum L. aerial part; Fragaria vesca L leaves; Plantago major L. leaves; Ribes nigrum L. leaves; Saccharina latissima (L.) C.E.Lane, C.Mayes, Druehl \& G.W.Saunders thallus; Linum usitatissimum L. seeds; Phaseolus vulgaris L. pericarp; Vaccinium myrtillus L. leaves; (3:2:2:2:3:1:3:3:2:2:5:7)

12.2 "Myrphasinum" Vaccinium myrtillus $\mathrm{L}$. shoots; Phaseolus vulgaris L. pericarp; Rosa spp. ${ }^{9}$ fruits; Urtica dioica L. leaves; Plantago major L. leaves; Matricaria chamomilla L. flowers;

Calendula officinalis L. flowers; Leonurus spp ${ }^{d}$ aerial part; Hypericum spp. ${ }^{e}$ aerial part; Achillea millefolium L. aerial part; Glycyrrhiza glabra L roots; Inula helenium L. roots; (2:2:1:1:1:1:1:1:1:1:1:1)
Infusion; 2-3 table spoons in $500 \mathrm{ml}$ of boiling water, maceration $12 \mathrm{~h}$ in thermos $66 \mathrm{ml}$ (warm) 3 times a Diabetes day 20-30 min before eating

Infusion; $10 \mathrm{~g}$ in $400 \mathrm{ml}$ of $100 \mathrm{ml} 2-3$ times a day boiling water 30 min before eating for 20-30 days
Ladynina and Morozova (1987), Ladynina and Morozova (1990); Lisitsyn and Molodozhnikova (1989); Lager (1991); Lager (2002); Efimov and Shcherbak (1993); Sinyakov (1999); Blinov (2000); Ladynina (2005); Nazina (2006); Bogdanova and Bashkirova (2010);

Mild forms of diabetes

Turishchev (2000); Dremova et al. (2003); Belodubrovskaya et al. (2004); Turishchev (2005); Davydovich et al. (2008); Ruzhenkova (2014)

\section{3 plants}

13.1 Avena sativa L. aerial part; Capsella bursa-pastoris (L.) Medik. aerial part; Cyanus segetum Hill flowers; Frangula alnus Mill. bark; Laurus nobilis L. leaves; Petroselinum crispum (Mill.) Fuss aerial part; Phaseolus vulgaris L. pericarp; Pimpinella anisum L. fruits; Rumex confertus Willd. roots; Syringa vulgaris L. buds; Taraxacum campylodes G.E.Haglund. roots; Tilia cordata Mill. flowers; Vaccinium myrtillus $L$. leaves ; (1:1:1:1:1:1:1:1:1:1:1:1:1)

13.2 Alchemilla xanthochlora Rothm. leaves; Arctostaphylos uva-ursi (L.) Spreng. leaves; Centaurium erythraea Rafn aerial part; Dioscorea spp. root; Helichrysum arenarium (L.) Moench flowers; Juniperus communis L. fruits; Mentha $\times$ piperita $L$. aerial part; Morus alba L. leaves; Plantago major L. leaves; Ribes nigrum L. leaves; Saccharina latissima (L.) C.E.Lane, C.Mayes, Druehl \& G.W.Saunders thallus; Vaccinium vitis-idaea L. leaves; Veronica officinalis L. aerial part; (1:1:1: 1:1:1:1:1:1:1:1:1:1)
Infusion; $12 \mathrm{~g}$ in $350 \mathrm{ml}$ of boiling water, $10 \mathrm{~min}$ in

$100 \mathrm{ml}$ (warm) 3 times a

Diabetes day 10 min before eating

boil water bath, maceration $2 \mathrm{~h}$ in thermos

Infusion; $12 \mathrm{~g}$ in $350 \mathrm{ml}$ of boiling water, $10 \mathrm{~min}$ in boil water bath, maceration $2 \mathrm{~h}$ in thermos
$100 \mathrm{ml}$ (warm) 3 times a Diabetes day 10 min before eating
Vinogradova et al. (2001)

Vinogradova et al. (2001) 
TABLE 1 | (Continued) The list of medical species used for the management of diabetes and related disorders in Russia.

\begin{tabular}{|c|c|c|c|c|c|}
\hline Code $^{a}$ & Plant name, part used /(proportion) & Method of preparation & $\begin{array}{c}\text { Recommended } \\
\text { dosage }\end{array}$ & Indication & Reference \\
\hline 13.3 & 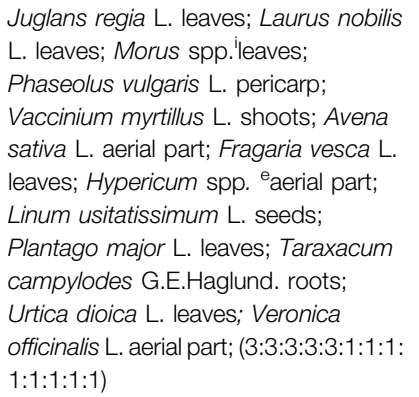 & $\begin{array}{l}\text { Infusion; } 15 \mathrm{~g} \text { in } 400 \mathrm{ml} \text { of } \\
\text { boiling water, } 15 \mathrm{~min} \text { in } \\
\text { boil water bath, } \\
\text { maceration } 2 \mathrm{~h} \text { in thermos }\end{array}$ & $\begin{array}{l}100 \mathrm{ml} 4 \text { times a day, } \\
15 \mathrm{~min} \text { before eating }\end{array}$ & Diabetes & Vinogradova et al. (2001) \\
\hline
\end{tabular}

\section{4 plants}

14.1 Achillea millefolium L. aerial part; Betula spp. 'leaves; Matricaria chamomilla L. flowers; Equisetum arvense L. aerial part; Foeniculum vulgare Mill. fruits; Frangula alnus Mill. bark; Glycyrrhiza glabra L. roots; Hypericum spp. ${ }^{e}$ aerial part; Juniperus communis L. fruits; Linum usitatissimum L. seeds; Melissa officinalis L aerial part; Petroselinum crispum (Mill.) Fuss roots; Ribes nigrum L. leaves; Urtica dioica L. leaves; (1:1:1: 1:1:1:1:1:1:1:1:1:1:1)

14.2 Anethum graveolens L. fruits; Crataegus spp. ${ }^{f}$ fruits; Fragaria vesca L. leaves; Helichrysum arenarium (L.) Moench flowers; Laurus nobilis L. leaves; Linum usitatissimum L. seeds; Mentha $\times$ piperita $L$. aerial part; Orthosiphon aristatus (Blume) Miq. shoots; Polygonum aviculare L. aerial part; Prunus spinosa L. flowers; Rheum palmatum L. roots; Rosa spp. ${ }^{9}$ fruits; Salvia officinalis L. leaves; Sorbus aucuparia L. fruits; $(1: 1: 1: 1: 1: 1: 1: 1: 1: 1$ : $1: 1: 1: 1)$

14.3 Juglans regia L. leaves; Plantago major L. seeds; Syringa vulgaris L. buds; Phaseolus vulgaris L. pericarp; Vaccinium myrtillus L. shoots; Arctium spp. 'broots; Taraxacum campylodes G.E.Haglund. roots; Ribes nigrum L. leaves; Helichrysum (arenarium (L.) Moench flowers; Betula spp. 'leaves; Urtica dioica L. leaves; Hypericum spp. eaerial part; Equisetum arvense L. aerial part; Fragaria vesca L. aerial part; (3:1: $1: 4: 4: 4: 4: 2: 2: 2: 2: 2: 2: 2)$

14.4 Laurus nobilis L. leaves; Vaccinium myrtillus L. shoots; Phaseolus vulgaris L. pericarp; Galega officinalis L. aerial part; Elymus repens(L.)

Couldrhizomes; Centaurium erythraea Rafn aerial part; Melissa officinalis L aerial part; Rosa spp. Ifruits; Glycyrrhiza glabra L. roots; Vaccinium vitis-idaea L. leaves; Betula spp. 'leaves; Linum usitatissimum L. seeds; Avena sativa L. aerial part; Trifolium pratense L.

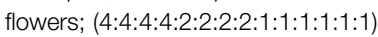

Infusion; $15 \mathrm{~g}$ in $500 \mathrm{ml}$ of boiling water, $15 \mathrm{~min}$ in boil water bath,

maceration $2 \mathrm{~h}$ in thermos

$100 \mathrm{ml} 4-5$ times a day

Obesity

Vinogradova et al. (2001) 20 min before eating
Infusion; $10 \mathrm{~g}$ in $500 \mathrm{ml}$ of cold water, maceration $4 \mathrm{~h}$ at room temp., boil 3 min, maceration $1 \mathrm{~h}$ in thermos

Infusion; $15 \mathrm{~g}$ in $400 \mathrm{ml}$ of boiling water, boil $3 \mathrm{~min}$, maceration $3 \mathrm{~h}$ in thermos before eating
$100 \mathrm{ml} 4-5$ times a day
Obesity
Vinogradova et al. (2001)
$100 \mathrm{ml} 4$ times a day 15 min before eating
Diabetes

Vinogradova et al. (2001)
Infusion; $15 \mathrm{~g}$ in $400 \mathrm{ml}$ of boiling water, $15 \mathrm{~min}$ in boik water bath, maceration $2 \mathrm{~h}$ in thermos.
$100 \mathrm{ml} 4$ times a day 15 min before eating
Diabetes
Vinogradova et al. (2001) 
TABLE 1 | (Continued) The list of medical species used for the management of diabetes and related disorders in Russia.

Code $^{\mathrm{a}}$ Plant name, part used/(proportion) Method of preparation

Recommended

dosage

\section{5 plants}

\section{1}

Arctium spp. 'broots; Galega officinalis L. aerial part; Laurus nobilis L. leaves; Phaseolus vulgaris L. pericarp; Vaccinium myrtillus L. shoots ; Vaccinium vitis-idaea L. leaves; Betula spp. ${ }^{C}$ leaves; Centaurium erythraea Rafn aerial part; Linum usitatissimum L. seeds; Rosa spp. ${ }^{\text {Ifruits; Syringa }}$ vulgaris L. buds; Melissa officinalis L. aerial part; Sorbus aucuparia L. fruits Trifolium pratense L. flowers; Viburnum opulus L. flowers; (3:3:3:3:3:3:2:2:2:2: $2: 1: 1: 1: 1)$

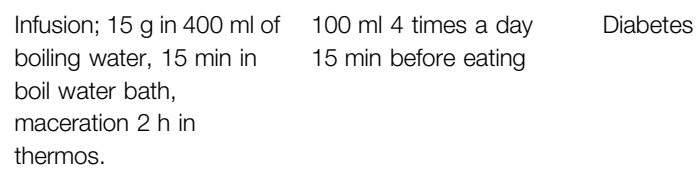

Indication

Reference

Code include number of plants and species number (for example 2.1 is mean 2 plants, species 1).

${ }^{b}$ Arctium spp. = Arctium lappa L., A. tomentosum Mill., A. minus (Mill.) Bernh.

${ }^{c}$ Betula spp. = Betula pendula Roth., B. pubescens Ehrh.

${ }^{d}$ Leonurus spp. = Leonurus quinquelobatus Gilib., L. cardiaca L

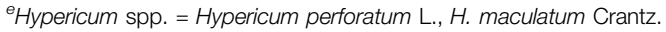

${ }^{f}$ Crataegus spp. = Crataegus laevigata (Poir.) DC. (C. oxyacantha sensu Pojark.), C. korolkovii L., Henry, C.chlorocarpa Lenne et C. koch (C. altaica (Lond.) Lange), C. dahurica Koehne ex Schneid., C. monogina Jacq., C.alemanniensis Cin., C. pentagyna Waldst. et Kit., C. orientobaltica Cin., C. curvisepala Lindm., C. $x$ curonica Cin.,C. $x$ dunensis Cin.

${ }^{g}$ Rosa spp. = Rosa majalis Herrm. (R. cinnamomea L.); R. acicularis Lindl.; R. davurica Pall.; R. beggeriana Schrenk.; R. fedtschenkoana Regel.; R. rugosa Thunb. et al.

${ }^{h}$ Quercus spp. = Quercus. robur L. and Q. petraea (Mattuschka) Liebl.

'Morus spp. = Morus alba L., M. nigra L.

The leaves of Vaccinium myrtillus were widely used in Europe for the treatment of diabetes for a long time before the discovery of insulin (Helmstädter and Schuster, 2010). The extract was considered a potent inhibitor of a-glucosidase, with an IC50 value not statistically significantly different from the IC50 of acarbose (Bljajić et al., 2017), and to decrease blood glucose (Cignarella et al., 1996) and glycated hemoglobin (Sidorova et al., 2017). An extract from the pericarp of Phaseolus vulgaris L. significantly decreases the levels of plasma triacylglycerol and low-density lipoprotein in the blood (Pari and Venkateswaran, 2004; Sidorova et al., 2017), lowers blood glucose and cholesterol in the blood, and inhibits a-amylase activity (Micheli et al., 2019). The normalization of lipid profiles and systemic antioxidant effects are also attributed to

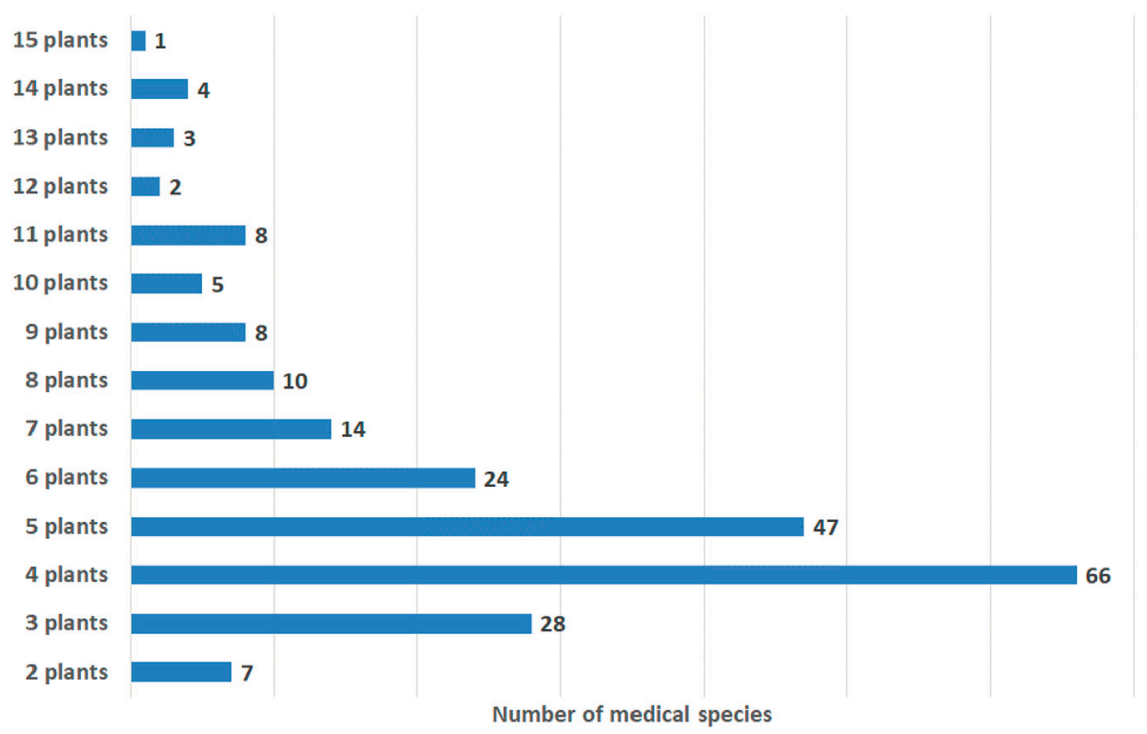

FIGURE 1 | Number of medical species according to the number of plants in the formulation. 
TABLE 2 | The most frequently mentioned binary combination of plants in medical species used for the treatment of diabetes.

\begin{tabular}{|c|c|c|c|c|c|c|c|c|c|c|c|}
\hline & $\begin{array}{l}\text { Vaccinium } \\
\text { myrtillus L. } \\
\text { leaves }\end{array}$ & $\begin{array}{c}\text { Phaseolus } \\
\text { vulgaris } \\
\text { L. } \\
\text { pericarp }\end{array}$ & $\begin{array}{c}\text { Urtica } \\
\text { dioica } \\
\text { L. } \\
\text { leaves }\end{array}$ & $\begin{array}{c}\text { Galega } \\
\text { officinalis } \\
\text { L. } \\
\text { aerial } \\
\text { part }\end{array}$ & $\begin{array}{c}\text { Taraxacum } \\
\text { campylodes } \\
\text { G.E.Haglund. } \\
\text { roots }\end{array}$ & $\begin{array}{c}\text { Fragaria } \\
\text { vesca } \\
\text { L. } \\
\text { leaves }\end{array}$ & $\begin{array}{c}\text { Rosa } \\
\text { spp. } \\
\text { fruits }\end{array}$ & $\begin{array}{l}\text { Arctium } \\
\text { spp. } \\
\text { root }\end{array}$ & $\begin{array}{c}\text { Vaccinium } \\
\text { vitis-idaea } \\
\text { L } \\
\text { leaves }\end{array}$ & $\begin{array}{l}\text { Hypericum } \\
\text { spp. }\end{array}$ & $\begin{array}{c}\text { Mentha } \\
\times \\
\text { piperita } \\
\text { L } \\
\text { leaves }\end{array}$ \\
\hline $\begin{array}{l}\text { Phaseolus } \\
\text { vulgaris L. } \\
\text { pericarp }\end{array}$ & 40 & & & & & & & & & & \\
\hline $\begin{array}{l}\text { Urtica dioica L. } \\
\text { leaves }\end{array}$ & 26 & 17 & & & & & & & & & \\
\hline $\begin{array}{l}\text { Galega officinalis } \\
\text { L. aerial part }\end{array}$ & 22 & 17 & 14 & & & & & & & & \\
\hline $\begin{array}{l}\text { Taraxacum } \\
\text { campylodes } \\
\text { G.E.Haglund. } \\
\text { roots }\end{array}$ & 21 & 19 & 19 & 9 & & & & & & & \\
\hline $\begin{array}{l}\text { Fragaria vesca L. } \\
\text { leaves }\end{array}$ & 14 & 8 & 8 & 4 & 5 & & & & & & \\
\hline Rosa spp. fruits & 16 & 10 & 6 & 4 & 4 & 10 & & & & & \\
\hline $\begin{array}{l}\text { Arctium spp. } \\
\text { roots }\end{array}$ & 15 & 9 & 5 & 5 & 2 & 4 & 8 & & & & \\
\hline $\begin{array}{l}\text { Vaccinium vitis- } \\
\text { idaea L. leaves }\end{array}$ & 13 & 8 & 5 & 10 & 2 & 4 & 7 & 8 & & & \\
\hline Hypericum spp. & 15 & 12 & 8 & 4 & 7 & 8 & 9 & 5 & 4 & & \\
\hline $\begin{array}{l}\text { Mentha } \times \\
\text { piperita } L \text {. leaves }\end{array}$ & 12 & 6 & 2 & 4 & 4 & 3 & 10 & 5 & 4 & 7 & \\
\hline $\begin{array}{l}\text { Linum } \\
\text { usitatissimum L. } \\
\text { seeds }\end{array}$ & 11 & 11 & 4 & 2 & 5 & 5 & 9 & 5 & 5 & 5 & 3 \\
\hline
\end{tabular}

this plant by other scientists (Venkateswaran et al., 2002; Helmstädter, 2010; Almuaigel et al., 2017).

The next most popular binary combination includes leaves of Vaccinium myrtillus L. and leaves of Urtica dioica L. (noted in 26 medical species). In addition to Vaccinium myrtillus L., the extract from the leaves of Urtica dioica L. reduces glycemia, potentiates the activity of insulin, enhances the utilization of glucose (El Haouari and Rosado, 2019), protects pancreatic $\beta$-cells (Golalipour and Khori, 2007), inhibits intestinal glucose absorption (Bnouham, et al., 2003), and shows total cholesterollowering activity (Avci et al., 2006). Eight weeks of treatment of patients with type 2 diabetes with Urtica dioica extract resulted in reductions in plasma glucose, triglycerides, and liver serum glutamic-pyruvic transaminase. Meanwhile, NO and superoxide markedly increased (Behzadi et al., 2016).

The combination of the leaves of Vaccinium myrtillus L. and aerial parts of Galega officinalis L. is described for 22 medical species. Complimentarily to Vaccinium myrtillus L., the extract from Galega officinalis L. reduces blood glucose, promotes the recovery of pancreatic $\beta$-cells (Sabeva et al., 2004; Shojaee et al., 2015), increases insulin-stimulated glucose uptake, activates peroxisome proliferator-activated receptor $(\operatorname{PPAR} \gamma)$ (Christensen et al., 2009), normalizes neutrophils, reduces lymphoblast numbers, and inhibits the apoptosis of lymphocytes, which prevents the development and progression of diabetic complications (Nagalievska et al., 2018). Galega officinalis L. is a world-renowned herbal lineage containing metformin (Bailey, 2017). It should be noted that the efficacy of the binary combination of extracts of Vaccinium myrtillus L. and Galega officinalis L. was confirmed in vivo. A dry extract of this combination $(50 \mathrm{mg} / \mathrm{kg}$ ) was intragastrically administered to rats with streptozotocin (STZ)-induced diabetes. After 21 days of treatment, histological examination evidenced the recovery of degenerative and focal necrobiotic changes in the parenchymatous structures of the liver and kidneys and their blood flow caused by STZ (Kurylo et al., 2018). In another study, the same combination of extracts was administered intragastrically to rats with STZ-induced diabetes for 28 days. After 7 days of treatment, blood glucose was decreased by $69 \%$ compared with control, while after 28 days of treatment, blood glucose was decreased by $25 \%$ compared with control. A positive effect of the combination was also observed in the oral glucose tolerance test (OGTT) (Kurylo et al., 2020). The rationality of the Vaccinium myrtillus L. and Galega officinalis L. combination was confirmed in a number of experiments by Achilov (2020). A screening study of the individual extracts (Vaccinium myrtillus L. $(50 \mathrm{mg} / \mathrm{kg})$ and Galega officinalis L. $(70 \mathrm{mg} / \mathrm{kg})$ ) and a combination at $50 \mathrm{mg} / \mathrm{kg}$ showed that, in OGTT in rats, the combination of the extracts decreased glucose more effectively than the individual extracts. The strongest effect was observed at $60 \mathrm{~min}$. Studies on models of epinephrine-induced hyperglycemia in rats, alloxan-induced diabetes in rats, and dithizone-induced diabetes in rabbits showed hypoglycemic activity of the combined extract at $60 \mathrm{mg} / \mathrm{kg}$ (Achilov, 2020). 
TABLE 3 | Complimentary mechanisms of most often used medicinal plants in binary combination in medical species used for the treatment of diabetes.

\begin{tabular}{|c|c|c|}
\hline $\begin{array}{l}\text { Medicinal plant and } \\
\text { part used }\end{array}$ & Mechanisms & References \\
\hline Fragaria vesca L. leaves & $\begin{array}{l}\text { Decrease of total cholesterol, triglycerides, low- and high-density } \\
\text { lipoproteins, normalization in antioxidant system (decrease of } \\
\text { malondialdehyde and increase of superoxide dismutase); inhibition of } \\
\text { a-glucosidase and a-amylase enzyme activity; reduce blood glucose level. }\end{array}$ & Tassa et al. (2012), Takács et al. (2020) \\
\hline Rosa spp. fruits & $\begin{array}{l}\text { Reduce blood glucose level, regulate lipid metabolism by inhibiting fat } \\
\text { accumulation (mainly visceral), decrease serum triglycerides, regeneration } \\
\text { of pancreas } \beta \text {-cells, increase expression of insulin-dependent genes Gck } \\
\text { and Ptp1b. }\end{array}$ & $\begin{array}{l}\text { Ninomiya et al. (2007), Orhan et al. (2009), Taghizadeh et al. (2016), } \\
\text { Fattahi et al. (2017), Bahrami et al. (2020) }\end{array}$ \\
\hline Arctium spp. roots & $\begin{array}{l}\text { Decrease of blood glucose, increase of insulin synthesis, suppression of } \\
\text { lipid synthesis by activating } 5^{\prime} \text {-adenosine monophosphate activated } \\
\text { protein kinase, regulated the expression of sterol regulatory element- } \\
\text { binding protein-1 and stearoyl-CoA desaturase. }\end{array}$ & Kuo et al. (2012), Ahangarpour et al. (2017), Chen et al. (2020) \\
\hline $\begin{array}{l}\text { Vaccinium vitis-idaea } \mathrm{L} \text {. } \\
\text { leaves }\end{array}$ & $\begin{array}{l}\text { Decrease of blood glucose, increase of insulin synthesis, decrease of } \\
\text { triglycerides, and high-density lipoproteins. }\end{array}$ & Barnaulov (2008), Zagayko et al. (2016) \\
\hline Hypericum spp. & $\begin{array}{l}\text { Reduce blood glucose level, inhibit pancreatic lipase, fat accumulation } \\
\text { reduce hypercholesterolemia, lowered total cholesterol and low-density } \\
\text { cholesterol, triglycerides, improved the insulin sensitivity, reduce } \\
\text { expression of Dgat1, ColV, andLp1 genes involved in the biosynthesis of } \\
\text { triglycerides }\end{array}$ & $\begin{array}{l}\text { Arokiyaraj et al. (2011), Husain et al. (2011), Hernández-Saavedra } \\
\text { et al. (2016), Tokgöz and Altan (2020) }\end{array}$ \\
\hline $\begin{array}{l}\text { Mentha } \times \text { piperita } L \\
\text { leaves }\end{array}$ & $\begin{array}{l}\text { Decrease of serum glucose, cholesterol, triglycerides, very low density } \\
\text { lipoprotein, low density lipoprotein. Increase the high density lipoprotein } \\
\text { cholesterol levels; inhibit glucosidase and tyrosinase }\end{array}$ & $\begin{array}{l}\text { Barbalho et al. (2011), Bayani et al. (2017), Pavlić et al. (2021), } \\
\text { Zeljković et al. (2021) }\end{array}$ \\
\hline $\begin{array}{l}\text { Linum usitatissimum L. } \\
\text { seeds }\end{array}$ & $\begin{array}{l}\text { Decrease blood glucose and polyphagia, control of lipid peroxidation } \\
\text { (thiobarbituric acid-reactive substances) and antioxidant enzymes } \\
\text { (glutathione peroxidase, superoxide dismutase, and catalase), inhibit } \\
\text { glucosidase and a-amylase }\end{array}$ & Bhat et al. (2011), Bouzghaya et al. (2020) \\
\hline
\end{tabular}

Roots of Taraxacum campylodes G.E. Haglund. in combination with the leaves of Vaccinium myrtillus L. are contained in 21 medical species; the former in combination with the leaves of Urtica dioica L. are used in 19 medical species, and the same in combination with the pericarp of Phaseolus vulgaris L. are used in 19 medical species (Table 2). The leaves of Taraxacum campylodes G.E. Haglund. are also used in binary combinations with the leaves of Vaccinium myrtillus L. and leaves of Urtica dioica L. The Taraxacum campylodes G.E. Haglund. root extract inhibits adipogenesis, regulates lipid metabolism by inhibiting fat accumulation, increases lipolysis, and normalizes cholesterol and triglyceride levels (GarcíaCarrasco et al., 2015). The leaf extract inhibited pancreatic lipase in vitro and in vivo, reduced triglyceride levels in the plasma of mice (Zhang et al., 2008), and stimulated the release of insulin in pancreatic $\beta$-cells (Hussain et al., 2004).

All the other binary combinations are used in fewer than $10 \%$ of the medical species discussed in this review. Therefore, we summarize the mechanisms of activities of the other plants cited in Table 2 separately in Table 3. The above-mentioned literature data suggest that binary combinations provide additive/ synergistic effects.

Notably, the binary combinations of the leaves of Vaccinium myrtillus L. and pericarp of Phaseolus vulgaris L.; leaves of Vaccinium myrtillus L. and roots of Arctium spp.; and roots of Taraxacum campylodes G.E. Haglund. and leaves of Mentha $\times$ piperita L. occur as self-sufficient medical species (Table $\mathbf{1}$ ).

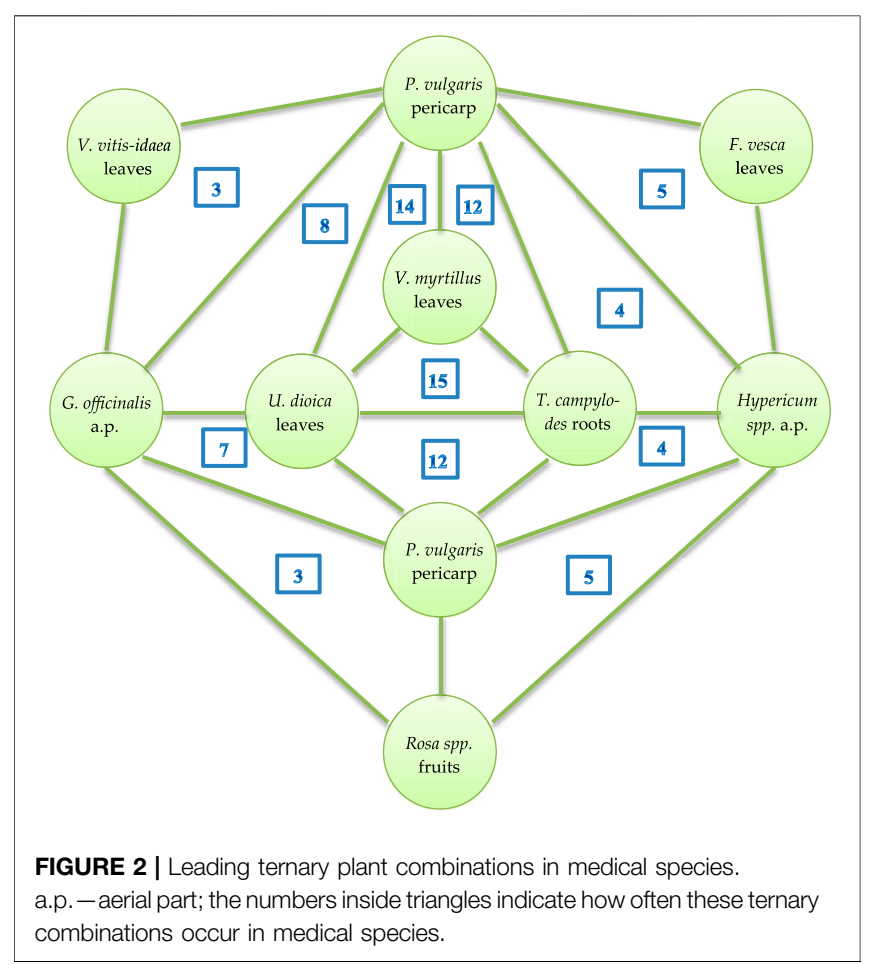

Deeper analysis of all the medical species allowed us to identify leading ternary plant combinations, which are presented in Figure 2. It is not surprising that 
TABLE 4 | The active compounds from medicinal plants most often used in combinations in medical species and probability of predicted antidiabetic mechanisms assitiated with these compounds.

\begin{tabular}{|c|c|c|c|c|}
\hline Medicinal plant/ (abbreviation) & Active compound & Mechanism of action & $\mathbf{P}_{\mathbf{a}}$ & $\mathbf{P}_{\mathbf{i}}$ \\
\hline \multirow[t]{4}{*}{ Vaccinium myrtillus L. (VM) } & Isoorientin & Antidiabetic & 0.806 & 0.005 \\
\hline & Vitexin-2"-rhamnosid & a-glucosidase inhibitor & 0.854 & 0.001 \\
\hline & & Antidiabetic & 0.767 & 0.005 \\
\hline & Inositol & Sugar-phosphatase inhibitor & 0.961 & 0.002 \\
\hline \multirow[t]{3}{*}{ Phaseolus vulgaris L. (PV) } & Isoorientin & Antidiabetic & 0.806 & 0.005 \\
\hline & Myricetin & Lipid peroxidase inhibitor & 0.836 & 0.003 \\
\hline & & $\beta$-glucuronidase inhibitor & 0.679 & 0.005 \\
\hline \multirow[t]{3}{*}{ Eleutherococcus senticosus (Rupr. \& Maxim.) Maxim. (ES) } & Syringin & Antidiabetic & 0.684 & 0.007 \\
\hline & & Hypolipemic & 0.674 & 0.016 \\
\hline & Eleutheroside E & Sugar-phosphatase inhibitor & 0.887 & 0.005 \\
\hline \multirow[t]{4}{*}{ Hypericum perforatum L. (HP) } & Hyperoside & Antidiabetic & 0.661 & 0.008 \\
\hline & & Lipid peroxidase inhibitor & 0.976 & 0.002 \\
\hline & & Sugar-phosphatase inhibitor & 0.874 & 0.006 \\
\hline & & a-glucosidase inhibitor & 0.842 & 0.001 \\
\hline \multirow[t]{7}{*}{ Aralia elata (Miq.) Seem (AE) } & Araloside A & Antidiabetic & 0.639 & 0.009 \\
\hline & & Hypolipemic & 0.955 & 0.003 \\
\hline & & Insulin promoter & 0.753 & 0.004 \\
\hline & Araloside B & Lipid peroxidase inhibitor & 0.969 & 0.002 \\
\hline & & Hypolipemic & 0.953 & 0.003 \\
\hline & & a-glucosidase inhibitor & 0.932 & 0 \\
\hline & Araloside C & Hypolipemic & 0.952 & 0.003 \\
\hline \multirow[t]{3}{*}{ Fragaria vesca L. (FV) } & Taxifolin-3-O-arabinofuranoside & Antidiabetic & 0.617 & 0.011 \\
\hline & & Lipid peroxidase inhibitor & 0.95 & 0.002 \\
\hline & & Antihypercholesterolemic & 0.901 & 0.003 \\
\hline \multirow[t]{3}{*}{ Urtica dioica L. (UD) } & 2-O-caffeoylmalic acid & Lipid metabolism regulator & 0.836 & 0.005 \\
\hline & Quercetin p-coumaroyl glucoside & Lipid peroxidase inhibitor & 0.978 & 0.002 \\
\hline & & a-glucosidase inhibitor & 0.729 & 0.001 \\
\hline \multirow[t]{4}{*}{ Galega officinalis L. (GO) } & Galegine & Sugar-phosphatase inhibitor & 0.632 & 0.047 \\
\hline & & Glucose oxidase inhibitor & 0.691 & 0.025 \\
\hline & Phytol & Lipid metabolism regulator & 0.828 & 0.005 \\
\hline & & Hypolipemic & 0.68 & 0.015 \\
\hline Taraxacum campylodes G.E.Haglund. (TC) & Taraxacin & $\beta$-glucuronidase inhibitor & 0.619 & 0.011 \\
\hline \multirow[t]{2}{*}{ Rosa spp. (Rsp) } & Lycopene & Sugar-phosphatase inhibitor & 0.794 & 0.017 \\
\hline & & Lipid metabolism regulator & 0.880 & 0.004 \\
\hline Arctium spp. (Asp) & Arctigenic acid & Insulin promoter & 0.579 & 0.017 \\
\hline \multirow[t]{2}{*}{ Vaccinium vitis-idaea L. (WI) } & Hydroquinone & Sugar-phosphatase inhibitor & 0.906 & 0.004 \\
\hline & & Inulinase inhibitor & 0.690 & 0.004 \\
\hline \multirow[t]{2}{*}{ Mentha × piperita L. (MP) } & Menthol & Insulin promoter & 0.773 & 0.004 \\
\hline & & Sugar-phosphatase inhibitor & 0.804 & 0.016 \\
\hline \multirow[t]{2}{*}{ Linum usitatissimum L. (LU) } & Gallic acid & Sugar-phosphatase inhibitor & 0.941 & 0.003 \\
\hline & & Glucan endo-1.6-beta-glucosidase inhibitor & 0.933 & 0.002 \\
\hline
\end{tabular}

the leaves of Vaccinium myrtillus L., pericarp of Phaseolus vulgaris L., roots of Taraxacum campylodes G.E. Haglund., leaves of Urtica dioica L., and aerial parts of Galega officinalis L. are principal members of the ternary combinations.

\section{In Silico Probability of Antidiabetic Activity for Principal Compounds Identified in Selected Plants}

The progress in computer science in symbiosis with modern pharmacology has led to the active implementation of computerbased prognosis for the activity of herb-derived compounds. Using an in silico approach, we analyzed the probability of antidiabetic activity for the principal compounds identified in the plants most often mentioned in binary and ternary combinations.
The prediction was performed using the free web resource PASS Online (Prediction of Activity Spectra for Substances). The prediction is based on an analysis of the structure and biological activity relationships for more than 300,000 organic compounds (Filimonov et al., 2014). Table 4 includes the prediction results for the antidiabetic efficacy of active compounds from selected medicinal plants with appropriate probability values: the likelihood of the given activity being revealed $(\mathrm{Pa})$ or not revealed $(\mathrm{Pi})$. If $\mathrm{Pa}>0.5$, the substance is very likely to exhibit the activity (Lagunin et al., 2000).

The predicted $\mathrm{Pa}$ values for the active compounds identified in the most frequently used combinations of plants in medical species were over 0.5 and ranged from 0.619 for compounds from Fragaria vesca L. up to 0.976 for compounds from Hypericum perforatum L. (Table 4). The highest $\mathrm{Pa}$ values were found for compounds derived from Urtica dioica L., 


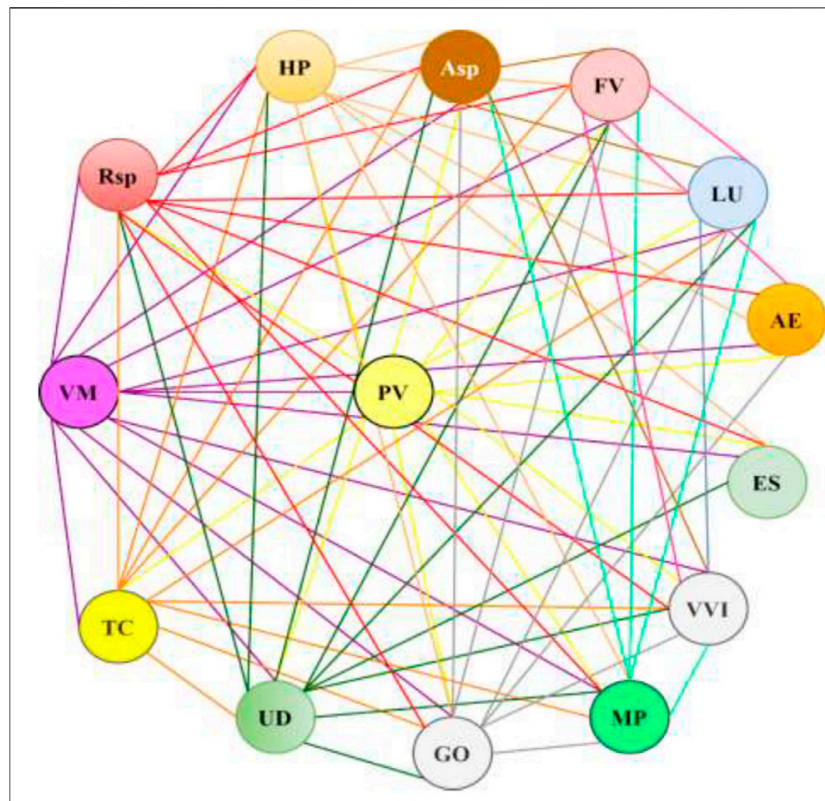

FIGURE 3 | The combinations of the medicinal plants with a high probability of antidiabetic effects in medical species. The plant abbreviations are presented in Table 4.

Hypericum perforatum L., Vaccinium myrtillus L., Fragaria vesca L., Linum usitatissimum L., and Vaccinium vitis-idaea L. $(\mathrm{Pa}>0.9)$. The diagram in Figure 3 demonstrates crosslinks between medicinal plants with a high probability of predicted antidiabetic effects (Table 4) used in medical species.

The calculated data support the rationality of the traditional use of medical species for the treatment of diabetes and its complications. Nevertheless, the chemical principles responsible for the observed effects are rarely studied. Except for the success story of metformin derived from Galega officinalis L., no other compounds are on the market. Systematic studies of the combinatory action of different plant decoctions/infusions, as well as plant-derived compounds, are needed.

\section{Specificity of Medical Species Used in Russia}

Several plants used in Russian traditional medicines for the treatment of diabetes and its complications are widely known in other countries. The leaves of Urtica dioica L., pericarp of Phaseolus vulgaris L., leaves of Vaccinium myrtillus L., and leaves and roots of Taraxacum campylodes G.E. Haglund. are among the most frequently used components for the management of diabetes by herbalists in Croatia (Končić and Bljajić, 2019). Phaseolus vulgaris is a well-known antidiabetic plant in the Ayurveda and Unani medicine systems (Ganesan and $\mathrm{Xu}$, 2017). It is widely used in medicine in Poland (Eabuda et al., 2017). The antidiabetic potential of Urtica dioica is well documented in Arabic traditional medicine (Said et al., 2008). Galega officinalis L. has been used for the treatment of diabetes in
Bulgaria (Petkov, 1982), Italy (Leporatti and Ivancheva, 2003), and Iran (Nowbandegani et al., 2015).

Unlike in other systems of medicine, the juices of some plants (the berries of Viburnum opulus L., Solanum tuberosum L., and Daucus sativus Roehl. and birch sap) have been used for preparing some medical species. Interestingly, eleven medical species contain seaweeds (Cystoseira barbata (Stackh.) C. Agardh; Saccharina latissima (L.) C.E.Lane, C.Mayes, Druehl \& G.W.Saunders; Fucus vesiculosus L.), and one species contains lichen (Cetraria islandica L.). It is noteworthy that several adaptogenic plants have been used in medical species. Besides the common properties of promoting the adaptability, resilience, and survival of living organisms under stress (Panossian et al., 2021), each adaptogen has some specific activity. In particular, Oplopanax elatus (Nakai) Nakai lowered blood glucose and increased insulin levels in vivo (Molokovskii et al., 2002). Glucose- and cholesterol-lowering effects, decreased glycosuria, and increased insulin levels were observed in diabetic patients after complex therapy with Oplopanax elatus (Nakai) Nakai (Klimakova and Kazmanm, 1962). Aralia elata (Miq.) Seem decreases blood glucose, inhibits insulin resistance, alleviates hyperlipidemia in vivo (Hwang et al., 2015), and improves blood glucose and lipid metabolism in humans (Abidov et al., 2006). The activity could be associated with aralosides ( $\mathrm{Pa}, 0.639-0.969$, Table 4). Eleutherococcus senticosus (Rupr. et Maxim.) Maxim. and its active compounds lowered blood glucose, increased glycogen levels, ameliorated insulin resistance, and increased insulin levels in vivo (Molokovskii et al., 2002; Niu et al., 2008; Ahn et al., 2013). The activity is associated with syringing and eleutheroside $\mathrm{E}$ ( $\mathrm{Pa}, 0.684$ and 0.887 ; Table 4). These adaptogens are not only used in medical species in traditional medicine but are included in the officinal medical species "Arfazetin" (Table 1).

\section{Principles for Compilation of Medical Species}

Due to the specific location of Russia, Russian herbal medicine has adopted the philosophy of Eastern traditional medicine and the pragmatic approach of Western medicine. One of the main principles for the compilation of the formulas used in traditional Chinese medicine is described in Shen-nong BenCao Jing. An effective formula should be based on a strong monarch, accompanied by a minister, assistant, and guide, which mimics a well-organized society (Xin et al., 2014; Xutian et al., 2014). However, this principle is difficult to follow in practice, due to the multiple symptoms of diseases and polyfunctionality of medicinal plants. Therefore, many formulas of TCM contain secrets that are not always explained by rationality (Wang et al., 2021).

The philosophy and conceptualization for the compilation of medical species in Russian medicine are not well described. After a comprehensive medical examination of a patient, a Russian phytotherapeutic doctor initially prescribes a basic medical species, which includes the plants that lower blood glucose. The binary and triple combinations emphasized in this review can be regarded as basic mixtures. Diabetes is often accompanied by obesity. Therefore, the basic mixture is fortified with plants reducing hypercholesterolemia. In the case of hypertension, the 
species include anti-hypertensive plants. The practical doctors also take into account the peculiarities of the gastrointestinal tracts of the patients. In this respect, medical species can include plants with astringent or laxative properties. To prevent allergic reactions, doctors recommend taking a basic mixture for a week and then continuously increasing the number of plants in the medical species one by one. Plants with antiallergic properties are sometimes included in the mixtures (Kovaleva, 1972; Ladynina and Morozova, 1987; Ladynina and Morozova, 1990).

This approach could be illustrated by the following medical species frequently cited in the literature. The species 4.39 (Table 1) includes a synergistic combination of Vaccinium myrtillus L. and Galega officinalis L., which effectively decreases glucose levels (Achilov, 2020; Kurylo et al., 2020). These basic plants have antidiabetic properties. Frangula alnus Mill. acts as a laxative, and Betula pendula Roth. acts as diuretic (Belodubrovskaya et al., 2004). Another species, 4.40 (Table 1), besides the synergistic combination of Vaccinium myrtillus L. and Galega officinalis L., includes Phaseolus vulgaris L., which reduces plasma triacylglycerol and low-density lipoprotein, and lowers blood glucose and cholesterol (Pari and Venkateswaran, 2004; Sidorova et al., 2017; Micheli et al., 2019). These basic plants ensure the antidiabetic effect, while Mentha $\times$ piperita $\mathrm{L}$. additionally provides anti-hypertensive, antiallergic, and spasmolytic effects (Mahendran and Rahman, 2020). The species 5.10 (Table 1) comprises 5 plants. The power of the basic mixture of the synergistic combination of Vaccinium myrtillus L. and Galega officinalis L. and Phaseolus vulgaris L. is reinforced by Taraxacum campylodes G.E. Haglund., which inhibits adipogenesis and fat accumulation (García-Carrasco et al., 2015). Additionally, Urtica dioica L. potentiates the activity of insulin and enhances the utilization of glucose (El Haouari and Rosado, 2019). We understand that the interpretation of the rationality of the above-mentioned medical species compilation took into account the results of modern research. Apparently, the architects of the discussed medical species compiled them according to their own experience and knowledge. Similar to the TCM formulas, some Russian medical species also have secrets that are yet to be deciphered. Nevertheless, the knowledge and practical experience of Russian traditional medicine were successfully utilized for the development of medical species used in officinal medicine.

\section{Medical Species Used in Russian Officinal Medicine}

In Russia, medical species are part of officinal medicine. Although medical species are available as OTC products, consultations with phytotherapeutic doctors are helpful and will lead to more effective results. Among the 227 medical species discussed in this review, only two, "Arfazetin" and "Myrphasinum", are approved for use in officinal medicine. Both species are recommended in the mild form of diabetes. The medical species "Arfazetin" was developed in the All-Union Institute of Medicinal and Aromatic Plants and was approved for medicinal use in 1986 (Ferubko et al., 2016). “Arfazetin” comprises seven medicinal plants (Table 1). In 1992, the composition of species was revised. The roots of Aralia elata (Miq.) Seem (syn. Aralia mandshurica Rupr. et Maxim.) or roots and rhizomes of Oplopanax elatus (Nakai) Nakai were excluded. Instead of these plants, the roots and rhizomes of Eleutherococcus senticosus (Rupr. et Maxim.) Maxim. were included in species at the same rate. A new species was named "Arfazetin-E". Both these species ("Arfazetin" and "Arfazetin-E") are now included in the State register (2021).

"Myrphasinum" was developed in 1985 by scientists from the first Moscow medical institute, named after I.M. Sechenov (Fas'kov et al., 1991), and was approved for medicinal use in 1991. The composition is complicated and includes the 12 medicinal plants (Table $\mathbf{1}$ ).

According to the regulatory requirements of the USSR/Russia, medical species are subject to preclinical and clinical evaluations of safety and efficacy.

\section{Preclinical and Clinical Data}

The efficacy of "Arfazetin" was studied in several experiments in vivo. Rats with alloxan-induced diabetes were administered an infusion of "Arfazetin" (10 ml/kg per day, orally) five days before alloxan injection and seven days after injection. Distilled water was administered in the control group. The blood glucose level, elevated by alloxan, was decreased in "the Arfazetin"-treated rats by 24 and $38 \%$ when compared with control on the third and seventh days, respectively, after alloxan injection. The total cholesterol, creatinine, and malondialdehyde in the blood and liver were decreased by $27,37,30$, and $30 \%$, respectively, compared with the control group on the seventh day after treatment. The treatment of rats with "Arfazetin" led to an increase in serum immunoreactive insulin and C-peptide after glucose load by 22 and 55\%, respectively, when compared with the control group (Azhunova et al., 2001). Similar results were observed in a prolonged study. The oral administration of "Arfazetin" ( $5 \mathrm{ml} / \mathrm{kg}$ of infusion) to rats with alloxan-induced diabetes over 30 days (7 days before alloxan induction and 23 days after injection) resulted in decreases in blood glucose of 46 and 39\%, respectively, compared with the control group on the 15 th and 30th days of the experiment. On Day 30, glycogen in the liver was increased by $17 \%$ when compared with the control group (Ishankulova et al., 2013). A further study by the same group evidenced the lipid-lowering properties of "Arfazetin" (infusion, $5 \mathrm{ml} / \mathrm{kg}$, orally). The total cholesterol in the blood decreased by $33 \%$ when compared with the negative control after 30 days of the treatment of rats with alloxan-induced diabetes. The levels of triglycerides and low- and high-density lipoproteins normalized and were equal to those in the intact group after 30 days of treatment with the infusion of "Arfazetin" (Ishankulova and Yuldasheva, 2019). In another study, the effects of "Arfazetin" on energy metabolism in rats were reported. Rats with alloxan-induced diabetes were treated with the infusion of "Arfazetin" (10 ml/kg, orally, daily) for 21 days. The control group received the same volume of distilled water. The treatment of rats with "Arfazetin" resulted in a double increase in adenosine triphosphate production in the liver when compared with control, starting from the seventh day of the experiment. The 
concentration of lactic acid decreased by 1.7 fold, while the activity of pyruvate kinase increased by 1.5 fold when compared with the control group after 21 days of treatment. The authors suggest positive effects of "Arfazetin" on energy metabolism (Lemza et al., 2014). The blood glucose in rats with alloxan-induced diabetes was decreased by 3.2 fold when compared with control at $3 \mathrm{~h}$ after the intragastric administration of a dry extract of the medical species "Arfazetin" (1,200 ng/kg). The efficacy of the extract was equal to that of gliclazide $(50 \mathrm{mg} / \mathrm{kg}$, intragastric administration) (Kvasova, 2011).

The acute and chronic toxicity of "Arfazetin" was studied in mice after oral administration. The $\mathrm{LD}_{50}$ for the dry extract of "Arfazetin" was $24 \mathrm{~g} / \mathrm{kg}$ (acute toxicity). No signs of toxicity were observed in the mice after 30 days of the administration of the "Arfazetin" infusion and dry extract at $1,200 \mathrm{mg} / \mathrm{kg}$ (there times a day every $4 \mathrm{~h}$ ) (Kvasova et al., 2010).

The antidiabetic potential of the medical species "Myrphasinum" was investigated in rats. Diabetes was modulated by the subcutaneous injection of alloxan. The glucose level in the blood was increased from $5.6 \mathrm{mmol} / \mathrm{L}$ (intact group) to $9.55 \mathrm{mmol} / \mathrm{L}$, body weights were decreased, and the rats had no appetite. The aqueous infusion of "Myrphasinum" ( $25 \mathrm{~g} / \mathrm{L})$ was administered to rats by an intragastric route at the dose of $5 \mathrm{ml} / \mathrm{kg}$ three times per day for two weeks. The control group was administered saline. The blood glucose in the treated group was decreased to $3.4 \mathrm{~mol} / \mathrm{L}$ (vs. an increase up to $10.7 \mathrm{ml} / \mathrm{L}$ in the control group) two weeks after the beginning of treatment. The body weights and appetite were improved. Meanwhile, $25 \%$ of the animals in the control group died. The animals were observed for five extra weeks after the end of treatment with "Myrphasinum". Three weeks after the end of treatment, the blood glucose in the treated group was equal to that in the intact group $(5.77 \mathrm{mmol} / \mathrm{L})$ and was stable until the fifth week (Grinkevich et al., 1997). In another study, outbred rats with alloxan-induced diabetes were orally administered $10 \mathrm{ml} / \mathrm{kg}$ of an infusion of "Myrphasinum" 3 times a day for three weeks. The control group received the same dose of normal saline. The treatment with "Myrphasinum" resulted in a statistically significant decrease in blood glucose by $26 \%$ compared with control. Glycogen in the liver and skeletal muscles was increased by 35 and $21 \%$ respectively, when compared with control and was equal to the level in an intact group (Dzhafarova, 2013). Subsequently, the efficacy of "Myrphasinum" in outbred rats with alloxan-induced diabetes was evaluated. The rats were treated orally with $10 \mathrm{ml} / \mathrm{kg}$ of an infusion of "Myrphasinum" 3 times a day. The control group received normal saline. Metformin ( $5 \mathrm{mg} / \mathrm{kg}$, 2 times a day) served as a positive control. The administration of "Myrphasinum" for 21 days did not affect the body weights of the rats, and no signs of toxicity were observed. The level of glucose in the "Myrphasinum"-treated group was decreased by $75 \%$ compared with control (vs. 59\% in the metformin group). The insulin and C-peptide levels were dramatically reduced by 3 and 3.3 fold, respectively, in rats after alloxan injection in those treated with "Myrphasinum" (vs. 1.5 and 1.7 fold increases, respectively, in the animals treated with metformin) (Jafarova and Garayev, 2013).

We have found, in the available literature, only a few publications about clinical trials with medical species. The efficacy of "Arfazetin" was studied in a group of 32 patients (18-65 years old) with types I and II diabetes mellitus. "Arfazetin" was prepared in the form of an aqueous infusion $(10 \mathrm{~g}$ in $400 \mathrm{ml})$ and administered in warm form at the dose of $1 / 3$ glass, three times a day, $30 \mathrm{~min}$ before meals, for one month. The patients with type I diabetes (12 persons) were administered "Arfazetin" in combination with an appropriate dose of insulin and diet. The five patients with a mild form of type II diabetes were administered "Arfazetin" in combination with an appropriate diet. The group of 15 patients with a moderate form of type II diabetes were administered "Arfazetin" in combination with an appropriate dose of hypoglycemic drugs and diet. In the patients with type I diabetes treated with "Arfazetin", a statistically significant decrease in blood glucose (by 38\%) was registered at $11.00 \mathrm{pm}$ when compared with 9.00 am of the same day. The effect was not cumulative. More pronounced results were observed in patients with type II diabetes. "Arfazetin" effectively ameliorated hyperglycemia. The doses of hypoglycemic drugs were reduced in 7 patients. In two patients, it was possible to maintain normal blood glucose levels without hypoglycemic drugs (Korotkova et al., 1988).

A "Myrphasinum" infusion was used in clinical praxis for the therapy of patients with and without diabetes decompensation. The treatment of patients with diabetes in the compensation stage resulted in statistically significant decreases in glucose by 15 and $44 \%$ in the blood and urine, respectively. Cholesterol and B-lipoproteins were decreased by 18 and $21 \%$, respectively. The effects of "Myrphasinum" in patients with diabetes in the decompensation stage were less pronounced (Fas'kov et al., 1991). However, the data provided in the inventor's certificate are limited and lacking other details.

The comparative efficacy of "Arfazetin" and "Myrphasinum" was studied in 57 patients with diabetes (22-76 years old) in an open clinical trial. The first group (26 persons) was treated with "Arfazetin", while the second group (31 persons) received "Myrphasinum". Basic therapy includes oral hypoglycemic drugs. "Myrphasinum" was considered more effective and resulted in a statistically significant decrease in blood glucose, surpassing “Arfazetin” in efficacy (Firsova et al., 1990). However, no more details were provided in this conference paper.

\section{CONCLUSION}

In this review, we analyze the compositions and potential of medical species used in Russian traditional and officinal medicine for the treatment of diabetes and related diseases. Several species besides medicinal plants contain fresh juices from berries, birch sap, and seaweeds. Another aspect of medical species is the presence of adaptogens. The philosophy and conceptualization for the compilation of medical species in Russian medicine are not well described. We have highlighted the most common binary and triple combinations of plants exploited in medical species. These combinations can be considered base mixes. Other plants are added to the mixtures to improve the efficacy, treat associated disorders, improve gastrointestinal function, prevent allergic reactions, etc. Obviously, Russian phytotherapeutic doctors 
compile polyherbal mixtures according to their own experience and knowledge. Modern studies of the mechanisms of action and predicted activities of the principal compounds from medicinal plants support the rationality of polyherbal mixtures. However, the mechanisms are not well studied and reported due to the limited number of compounds. Deeper investigations including gene expression will enable a better understanding of molecular mechanisms and targets. Although a few studies have evidenced possible additive/synergistic effects of herbal mixtures, additional investigations with calculations of synergistic or additive indices will assist in providing a scientific foundation for the wider use of medical species for the therapy of diabetes. Even though most medical species comprise mixtures of three to six plants, other species also deserve careful study. It appears to us that the species with seven or more plants have rationality that is difficult to explain and some secrets that are yet to be deciphered. On the other hand, modern good praxis rules require the identification of all the plants in medical species. An increase in plants in the mixture requires advanced techniques for quality control. Notably, two medical species approved for use in officinal medicine include 7 and 12 plants. The efficacy of these species was investigated in vivo. However, all the activities were proved using only one model of alloxan-induced

\section{REFERENCES}

Abidov, M. T., del Rio, M. J., Ramazanov, T. Z., Klimenov, A. L., Dzhamirze, S., and Kalyuzhin, O. V. (2006). Effects of Aralia Mandshurica and Engelhardtia Chrysolepis Extracts on Some Parameters of Lipid Metabolism in Women with Nondiabetic Obesity. Bull. Exp. Biol. Med. 141 (3), 343-346. doi:10.1007/ s10517-006-0167-3

Achilov, D. D. (2020). Hypoglycemic Properties of Phytobiabetol Obtained Based on Plants Vaccinium Myrtillus L., Galega Oficinalis L. Thesis of $\mathrm{PhD}$ dissertation. Tashkent, Uzbekistan. Available at: http://repository.tma.uz/jspui/handle/1/801.

Ahangarpour, A., Heidari, H., Oroojan, A. A., Mirzavandi, F., Nasr Esfehani, K., and Dehghan Mohammadi, Z. (2017). Antidiabetic, Hypolipidemic and Hepatoprotective Effects of Arctium Lappa Root's Hydro-Alcoholic Extract on Nicotinamide-Streptozotocin Induced Type 2 Model of Diabetes in Male Mice. Avicenna J. Phytomed. 7 (2), 169-179.

Ahn, J., Um, M. Y., Lee, H., Jung, C. H., Heo, S. H., and Ha, T. Y. (2013). Eleutheroside E, an Active Component of Eleutherococcus Senticosus, Ameliorates Insulin Resistance in Type 2 Diabetic Db/db Mice. Evid. Based Complement. Alternat. Med. 2013, 934183. doi:10.1155/2013/934183

Akamova, A. V., Nemyatykh, O. D., and Narkevich, I. A. (2017). Multiple View Marketing Analysis of the Russian Plant-Based Drugs Market. Drug Dev. registration 4 (21), 276-280.

Almuaigel, M. F., Seif, M. A., Albuali, H. W., Alharbi, O., and Alhawash, A. (2017). Hypoglycemic and Hypolipidemic Effects of Aqueous Extract of Phaseolus Vulgaris Pods in Streptozotocin-Diabetic Rats. Biomed. Pharmacother. 94, 742-746. doi:10.1016/j.biopha.2017.07.135

Anzar, M. A. (2013). Drugs Indicated for the Management of Ziabetus Shakri (Diabetes Mellitus) in Unani Medicine-An Overview. Int. Jour. Pharmamedix India 1 (3), 460-474.

Arai, Y.-C., Makino, I., Ikemoto, T., Saisu, H., Terajima, Y., and Owari, K. (2020). Kampo for the Treatment of Pain in Japan: A Review. Pain Ther. 9 (1), 161-170. doi:10.1007/s40122-020-00160-w

Arokiyaraj, S., Balamurugan, R., and Augustian, P. (2011). Antihyperglycemic Effect of Hypericum perforatum Ethyl Acetate Extract on Streptozotocin-Induced Diabetic Rats. Asian Pac. J. Trop. Biomed. 1 (5), 386-390. doi:10.1016/S22211691(11)60085-3 diabetes. Clinical trials were completed in small groups, and several details are not indicated in the reports. According to modern regulatory rules, additional pharmacological experiments and clinical trials are required for more detailed investigations of the mechanisms of action and the confirmation of efficacy. We believe that the scientifically based utilization of rich plant resources and knowledge of Russian herbal medicine can significantly contribute to the local economy as well as to the sectors seeking natural healing products.

\section{AUTHOR CONTRIBUTIONS}

AS, AA, and OP designed the study. AS, AA, ON, VL and OP collected information on the medical species. AS, AA, IM and OP conducted the literature search, extracted the data, and wrote the first draft. AA and MP collected information from web resources. $\mathrm{ON}, \mathrm{EF}$ and IN oversaw the research project, including checking the research work, reviewing, and interpreting the results. All authors are involved in reviewing and approval of the final manuscript.

Avci, G., Kupeli, E., Eryavuz, A., Yesilada, E., and Kucukkurt, I. (2006). Antihypercholesterolaemic and Antioxidant Activity Assessment of Some Plants Used as Remedy in Turkish Folk Medicine. J. Ethnopharmacol. 107 (3), 418-423. doi:10.1016/j.jep.2006.03.032

Azhunova., T. A., Lemza, S. V., and Linkhoyeva, E. I. (2001). Pharmacotherapeutic Efficacy of Complex Plant Remedy in Experimental Diabetes. Bull. East. Siberian Scientific Cent. Siberian Department Russ. Acad. Med. Sci. 1-2 (77), 104-108.

Bahrami, G., Miraghaee, S. S., Mohammadi, B., Bahrami, M. T., Taheripak, G., Keshavarzi, S., et al. (2020). Molecular Mechanism of the Anti-diabetic Activity of an Identified Oligosaccharide from Rosa Canina. Res. Pharm. Sci. 15 (1), 36-47. doi:10.4103/1735-5362.278713

Bailey, C. J. (2017). Metformin: Historical Overview. Diabetologia 60 (9), 1566-1576. doi:10.1007/s00125-017-4318-z

Balakirev, G. V., Gubanov, I. A., Kozina, E. M., Samokhina, E. B., Taranova, I. A., Tugunova, O., et al. (2010). Travnik. Encyclopedia of Medicinal Plants. G.A. Nepokoichitsky (editor-in-chief). 640. Moscow: Publishing House.

Banerjee, S., Bhattacharjee, P., Kar, A., and Mukherjee, P. K. (2019). LC-MS/ MS Analysis and Network Pharmacology of Trigonella Foenum-Graecum A Plant from Ayurveda against Hyperlipidemia and Hyperglycemia with Combination Synergy. Phytomedicine 60, 152944. doi:10.1016/ j.phymed.2019.152944

Barbalho, S. M., Damasceno, D. C., Spada, A. P. M., Silva, V. S. D., Martuchi, K. A., Oshiiwa, M., et al. (2011). Metabolic Profile of Offspring from Diabetic Wistar Rats Treated with Mentha piperita (Peppermint). Evidence-Based Complement. Altern. Med. 2011, 1-6. doi:10.1155/2011/430237

Barnaulov, O. D. (2008). The Comparative Estimation Influence of Drugs from Russian Flora Plants on Insuline and Glucose Blood Level in Alloxane-Diabetic Rats. Psychopharmacol. Biol. Narcol. 8 (3-4), 2484-2490.

Bayani, M., Ahmadi-Hamedani, M., and Jebelli Javan, A. (2017). Study of Hypoglycemic, Hypocholesterolemic and Antioxidant Activities of Iranian Mentha Spicata Leaves Aqueous Extract in Diabetic Rats. Iran J. Pharm. Res. 16 (Suppl. 1), 75-82.

Behzadi, A. A., Kalalian-Moghaddam, H., and Ahmadi, A. H. (2016). Effects of Urtica dioica supplementation on blood lipids, hepatic enzymes and nitric oxide levels in type 2 diabetic patients: A double blind, randomized clinical trial. Avicenna J. Phytomed. 6 (6), 686. 
Belodubrovskaya, G. A., Blinova, K. F., Vandyshev, V. V., Zhokhova, E. V., Klemper, A. V., Komarova, M. N., et al. (2004). Medicinal plant raw materials. Pharmacognosy: textbook. Editors G.P. Yakovlev and K. Blinova. St. Petersburg: Spezlit, 765. [in Russia].

Bhat, M., Zinjarde, S. S., Bhargava, S. Y., Kumar, A. R., and Joshi, B. N. (2011). Antidiabetic Indian Plants: a Good Source of Potent Amylase Inhibitors. Evidence-Based Complement. Altern. Med. 2011, 1-6. doi:10.1093/ecam/nen040

Blinov, V. A. (2000). Medicinal Plants for Diabetes Mellitus. Moscow: Publishing house Raduga.

Bljajić, K., Petlevski, R., Vujić, L., Čačić, A., Šoštarić, N., Jablan, J., et al. (2017). Chemical Composition, Antioxidant and a-glucosidaseinhibiting Activities of the Aqueous and Hydroethanolic Extracts of Vaccinium Myrtillus Leaves. Molecules 22 (5), 703. doi:10.3390/ molecules22050703

Bnouham, M., Merhfour, F. Z., Ziyyat, A., Mekhfi, H., Aziz, M., and Legssyer, A. (2003). Antihyperglycemic Activity of the Aqueous Extract of Urtica Dioica. Fitoterapia 74 (7-8), 677-681. doi:10.1016/s0367-326x(03) 00182-5

Bogdanova, O., and Bashkirova, N. A. (2010). The Big Book of Diabetics: Everything You Need to Know about Diabetes: Moscow: AST. St. Petersburg: Prime-Euroznak, 280. [In Russian].

Bouzghaya, S., Amri, M., and Homblé, F. (2020). Improvement of Diabetes Symptoms and Complications by an Aqueous Extract ofLinum usitatissimum (L.) Seeds in Alloxan-Induced Diabetic Mice. J. Med. Food 23 (10), 1077-1082. doi:10.1089/jmf.2019.0205

Brusenskaya, I. V., and Kaz'min, V. D. (2005). Treatment by Traditional Medicines. Rostov on the Don: Phoenix. [In Russian].

Bubenchikova, V. N., Bubenchikov, A. A., and Filippenko, N. G. (2003). Home Doctor Book: Phytotherapy in Your House. Moscow, RIPOL CLASSIC. [In Russian].

Centers for Disease Control and Prevention (2020). National Diabetes Statistics Report, 2020. Atlanta, GA: Centers for Disease Control and Prevention, US Department of Health and Human Services. Available at: https://www.cdc.gov/diabetes/pdfs/data/statistics/national-diabetesstatistics-report.pdf.

Chekina, N. A., Chukaev, S. A., and Nikolaev, S. M. (2010). Diabetes Mellitus: Possibilities of Pharmacotherapy Using Plant Remedies. Vestnik Buryatskogo Gos Universiteta 12, 71-78.

Chen, M., Xu, J., Wang, Y., Wang, Z., Guo, L., Li, X., et al. (2020). Arctium Lappa L. Polysaccharide Can Regulate Lipid Metabolism in Type 2 Diabetic Rats through the SREBP-1/SCD-1 axis. Carbohydr. Res. 494, 108055. doi:10.1016/ j.carres.2020.108055

Chirkov, A. I., and Seryi, V. S. (1993) Medical Species (Monography). Moscow: Medicine, 287. [In Russian].

Christensen, K. B., Minet, A., Svenstrup, H., Grevsen, K., Zhang, H., Schrader, E., et al. (2009). Identification of Plant Extracts with Potential Antidiabetic Properties: Effect on Human Peroxisome Proliferator-Activated Receptor (PPAR), Adipocyte Differentiation and Insulin-Stimulated Glucose Uptake. Phytother. Res. 23 (9), 1316-1325. doi:10.1002/ptr.2782

Cignarella, A., Nastasi, M., Cavalli, E., and Puglisi, L. (1996). Novel Lipid-Lowering Properties of Vaccinium Myrtillus L. Leaves, a Traditional Antidiabetic Treatment, in Several Models of Rat Dyslipidaemia: a Comparison with Ciprofibrate. Thromb. Res. 84, 311-322. doi:10.1016/s0049-3848(96)00195-8

Davydovich, M. G., Kostarev, O. V., and Farkhutdinov, R. G. (2008). Phytotherapy during Diabetes Mellitus. Ufa: Gilem.

Dedov, I. I., Shestakova, M. V., Vikulova, O. K., Zheleznyakova, A. V., and Isakov, M. A. (2018). Diabetes Mellitus in Russian Federation: Prevalence, Morbidity, Mortality, Parameters of Glycaemic Control and Structure of Glucose Lowering Therapy According to the Federal Diabetes Register, Status 2017. Diabetes mellitus 21 (3), 144-159. doi:10.14341/ DM20171S810.14341/dm9686

Dmitriev, A. I., Smolyansky, B. L., Kreknin, A. A., Mironets, T. N., and Vizgalova, I. I. (1994). Nutrition and phytotherapy for diabetes mellitus. Dnepropetrovsk: OGTIP "Dneprkniga" (40 p.) [In Russian]

Dontsov, V. V., and Dontsov, I. V. (2000). Medicinal Plants and Bee Products. More than 400 Recipes and Healing Species for All Diseases. Moscow: Tsentrpoligraf Publishing house, OOO "Polus". [In Russian].
Dremova, N. B., Bubenchikova, V. N., Drozdova, I. L., and Dzharu, M. (2003). Modern Medicines and Phytoterapy in the Complex Treatment and Prevention of Diabetes Mellitus : Educational and Methodical Manual. Kursk, Russia: Publishing house of the Kursk State Medical University. [In Russian].

Dzhafarova, R. A. (2013). Study of Action of Phytocomplex "Antidiabet" and "Mirfazin" to Sugar in Blood Plasma and Glycogen in Liver and Muscle Tissue. Bull. Russ. Mil. Med. Acad. 4, 172-174.

Efimov, A. S., and Shcherbak, A. V. (1993). Phytotherapy of Obesity and Diabet. Naukova dumka, Phoenix: Kiev. [In Russian].

Ekor, M. (2014). The Growing Use of Herbal Medicines: Issues Relating to Adverse Reactions and Challenges in Monitoring Safety. Front. Pharmacol. 4, 177. doi:10.3389/fphar.2013.00177

El Haouari, M., and Rosado, J. A. (2018). Phytochemical, Anti-diabetic and Cardiovascular Properties of Urtica Dioica L. (Urticaceae): A Review. Mrmc 19 (1), 63-71. doi:10.2174/1389557518666180924121528

Fas'kov, M. N., Nikolaev, A. V., Grinkevich, N. I., Balandina, I. A., Mamedov, L. A., Firsova, S. V., et al. (1991). Remedy for the Treatment of Diabetes. Inventor's certificate SU 1697820 A1-12.

Fattahi, A., Niyazi, F., Shahbazi, B., Farzaei, M. H., and Bahrami, G. (2017). Antidiabetic Mechanisms of Rosa Canina Fruits. J. Evid. Based Complement. Altern Med. 22 (1), 127-133. doi:10.1177/2156587216655263

Fedyukovich, N. I. (1998). Modern Hypoglycemic Drugs. Minsk: Universitetskoye. [In Russian].

Ferubko, E. V., Trumpe, T. E., Kurmanova, E. N., Kolkhir, V. K., Sidelnikova, G. F., Sidelnikov, N. I., et al. (2016). Human Use of FGBNU VILARs Phytopreparations. Probl. Biol. Med. Pharm. Chem. 19 (6), 11-15.

Filimonov, D. A., Lagunin, A. A., Gloriozova, T. A., Rudik, A. V., Druzhilovskii, D. S., Pogodin, P. V., et al. (2014). Prediction of the Biological Activity Spectra of Organic Compounds Using the PASS Online Web Resource. Chem. Heterocycl. Comp. 50 (3), 444-457. doi:10.1007/s10593-014-1496-1

Firsova, S. V., Kalinkina, M. A., and Koroleva, T. V. (1990). Comparative Clinical Evaluation of Antidiabetic Herbal speciesAbstracts of the All-union Scientific Conference of Young Scientists and Students Dedicated to the 225 Anniversiary of the First MMI Youth for Practical Health Care. Moscow, 65.

Ganesan, K., and $\mathrm{Xu}, \mathrm{B}$. (2017). Polyphenol-rich Dry Common Beans (Phaseolus vulgaris L.) and Their Health Benefits. Ijms 18 (11), 2331. doi:10.3390/ijms18112331

García-Carrasco, B., Fernandez-Dacosta, R., Dávalos, A., Ordovás, J., and Rodriguez-Casado, A. (2015). In Vitro Hypolipidemic and Antioxidant Effects of Leaf and Root Extracts of Taraxacum Officinale. Med. Sci. 3 (2), 38-54. doi:10.3390/medsci3020038

Ghadge, A. A., and Kuvalekar, A. A. (2017). Controversy of Oral Hypoglycemic Agents in Type 2 Diabetes Mellitus: Novel Move towards Combination Therapies. Diabetes Metab. Syndr. Clin. Res. Rev. 11, S5-S13. doi:10.1016/ j.dsx.2016.08.009

Golalipour, M. J., and Khori, V. (2007). The Protective Activity of Urtica Dioica Leaves on Blood Glucose Concentration and $\beta$-cells in Streptozotocin-Diabetic Rats. Pakistan J. Biol. Sci. 10 (8), 1200-1204. doi:10.3923/pjbs.2007.1200.1204

Grechanyi, I. A. (2013). Complete Reference Book of Medicinal Herbs and Healing Species: 600 Plants and species. Kharkiv: Family Leisure Club. Belgorod: Book Club "Family Leisure Club". [In Russian].

Grinkevich, N. I., Nikolaev, A. V., Firsova, S. V., Balandina, I. A., and Mamedov, L. A. (1997). The Study of Specific (Antidiabetic) Activity of Multicomponent Medical Species. Scientific Proceedings All-union Scientific Research Institute Pharmacy. Resource Pharmacognostic Study Medicinal Flora USSR Vol. XXV, 109-111.

Helmstädter, A. (2010). Beans and Diabetes: Phaseolus vulgaris Preparations as Antihyperglycemic Agents. J. Med. Food 13 (2), 251-254. doi:10.1089/ jmf.2009.0002

Helmstädter, A., and Schuster, N. (2010). Vaccinium myrtillus as an antidiabetic medicinal plant-research through the ages. Die Pharmazie 65 (5), 315-321. doi:10.1691/ph.2010.9402

Hernández-Saavedra, D., Pérez-Ramírez, I. F., Ramos-Gómez, M., Mendoza-Díaz, S., Loarca-Piña, G., and Reynoso-Camacho, R. (2016). Phytochemical Characterization and Effect of Calendula officinalis, Hypericum perforatum, and Salvia Officinalis Infusions on Obesity-Associated Cardiovascular Risk. Med. Chem. Res. 25 (1), 163-172. doi:10.1007/s00044-015-1454-1

Husain, G. M., Chatterjee, S. S., Singh, P. N., and Kumar, V. (2011). Hypolipidemic and Antiobesity-like Activity of Standardised Extract of Hypericum perforatum L. In Rats. ISRN Pharmacol. 2011, 1-7. doi:10.5402/2011/505247 
Hussain, Z., Waheed, A., Qureshi, R. A., Burdi, D. K., Verspohl, E. J., Khan, N., et al. (2004). The Effect of Medicinal Plants of Islamabad and Murree Region of Pakistan on Insulin Secretion from INS-1 Cells. Phytother. Res. 18 (1), 73-77. doi:10.1002/ptr.1372

Hwang, K.-A., Hwang, Y.-J., Kim, G. R., and Choe, J.-S. (2015). Extracts from Aralia Elata (Miq) Seem Alleviate Hepatosteatosis via Improving Hepatic Insulin Sensitivity. BMC Complement. Altern. Med. 15 (1), 1-9. doi:10.1186/ s12906-015-0871-5

IDF (2019). Diabetes Atlas. Available at: https://www.diabetesatlas.org/en/ (Accessed March 26, 2012).

Ishankulova, B. A., and Yuldasheva, U. P. (2019). Comparative Study of Antidiabetic Effect of Species "Marankhuch" and "Chordoru". Proceedings of the Conference "Medical Science of the XXI century - Looking towards the Future". Dushanbe 3, 69-70.

Ishankulova, B. A., Yuldasheva, U. P., and Urunova, M. V. (2013). Comparative Characteristics of Some Glucose-Lowering Plants and Anti-diabetic Collections of Tajikistan on Their Basis. Avicenna Bull. 1, 121125.

Jafarova, R. A., and Garayev, G. Sh. (2013). Comparative Pharmacological Study of the Effect of Collection of "Antidiabet" Galenical Medicines of mulberry Leaves and white and Grass clover Medicinalis on the Course of Alloxan Modeling Diabetes Mellitus. Amea-nın Xibırlıri. Biologiy Aelmlıriseriyası 68, 125-130.

Kiseleva, T. L., and Chauzova, A. V. (1999). Development of Algorithm for Preparation of Medical Species Based on the Traditional Recipes. Proceedings Book of the $3^{\text {rd }}$ International Congress Actual Problems of Creation of New Medicinal Preparations of Natural Origin. Pushkin: St-Petersburg, 211-215.

Kiyanova, I. V. (2005). Holy apothecary. Treatment of metabolic disorders (diabet, obesity, thyroid diseases). Moscow: Orthodox Brotherhood of the Holy Apostle John the Theologian. [In Russian].

Klimakova, A. I., and Kazmanm, M. A. (1962). Echinopanax Tincture in Complex Therapy of Patients with Diabetes Mellitus. Med. Preparations Plants, 250-258.

Kolesova, V. G., Marchenko, V. A., and Syrovezhko, N. V. (1998). Medicinal Plants: Myths and realityTraditional (Folk) Medicine in the Lens of Science. St. Petersburg: SPCPA Publishing. [In Russian].

Končić, M. Z., and Bljajić, K. (2019).Traditional Herbal Products Used for the Management of Diabetes in Croatia: Linking Traditional Use with a-Glucosidase-Inhibitory Activity Bioactive Food as Dietary Interventions for Diabetes. United States: Academic Press, 647-664.

Korodetsky, A. V. (2006). Korodetskii's green Apothecary against Diabetes: Basketplant. Stevia. Periwinkle. St. Petersburg: Piter, 92-96. [In Russian].

Korotkova, V. D., Perelygina, A. A., Trumpe, T. E., Sokolov, S. Ia., and Lobanova, A. M. (1988). [Arfazetin in the Treatment of Diabetes Mellitus]. Probl. Endokrinol (Mosk) 34 (4), 25-28.

Korsun, V. F., and Korsun, E. V. (2010) Phytotherapy: Tradition of Russian Herbalism. Moscow: Eksmo. [In Russian].

Korsun, V. F., Trumpe, T. E., Korsun, E. V., Ershov, N. V., and Ogrenich, N. A. (2016). Phytotherapy against Diabetes. Herbs of Life. Moscow: ZAO Tsentrpoligraph Publishing House. [In Russian].

Kovaleva, N. G. (1972). The Therapy with Plants. Moscow: Medizina. [In Russian].

Kukes, V. G. (1999). Phytotherapy with the Basics of Clinical Pharmacology. Moscow: Medizina. [In Russian].

Kuo, D.-H., Hung, M.-C., Hung, C.-M., Liu, L.-M., Chen, F.-A., Shieh, P.-C., et al. (2012). Body Weight Management Effect of Burdock (Arctium Lappa L.) Root Is Associated with the Activation of AMP-Activated Protein Kinase in Human HepG2 Cells. Food Chem. 134, 1320-1326. doi:10.1016/j.foodchem.2012.03.023

Kurylo, K., Budniak, L., Volska, A., Zablotskyy, B., and Klishch, I. (2020). Influence of Phytocompositions on Dynamics of Changes in Basal Glycemia and Glycemia in Oral Glucose Tolerance Test in Rats with Streptozotocin-Nicotinamide-Induced Diabetes Mellitus Type 2. Georgian Med. News (300), 112-116.

Kurylo, K. I., Klishch, I. M., Nebesna, Z. M., Furdela, M. Y., Volska, A. S., and Lytvynyuk, S. O. (2018). Histological Changes in Liver and Kidneys in Experimental Type 2 Diabetes Mellitus and its Correction by Administration of Galega Officinalis L. Phytocompositions. Womab 14 (63), 133-136. doi:10.26724/2079-8334-2018-1-63-133-136

Kvasova, T. M., Demidova, M. A., and Amosov, V. V. (2010). Assessment of Acute and Chronic Toxicity of Dry Extract of the Collection Arfazetin. Doctor -Aspirant 6 (43), 158-162.
Kvasova, T. M. (2011). Effect of Arfazetin Medical Species on the Experimental Diabetes Mellitus in Rats. Postgrad. Doctor 5 (48), 157-162. doi:10.26.724/20798334-2018-1-63-133-136

Ladynina, E. A., and Morozova, R. S. (1987). Phytotherapy. 1st ed. Moscow: Medizina. [In Russian].

Ladynina, E. A., and Morozova, R. S. (1990). Phytotherapy. 2nd ed. Moscow: Medizina. [In Russian].

Ladynina, E. A. (2005). The Wisdom of Herbs. Herbal Medicine and Homeopathy. Moscow: AiF-Print. [In Russian].

Lager, A. A. (2002). Herbs' Guide of Siberian Healer. Moscow: RIPOL CLASSIC. [In Russian].

Lager, A. A. (1991). Phytotherapy Diseases of the Digestive System. Krasnoyarsk: Publishing house of Krasnoyarsk University. [In Russian].

Lagunin, A., Stepanchikova, A., Filimonov, D., and Poroikov, V. (2000). PASS: Prediction of Activity Spectra for Biologically Active Substances. Bioinformatics 16 (8), 747-748. doi:10.1093/bioinformatics/16.8.747

Lavrenova, G. V., and Lavrenov, V. K. (2007). Complete Encyclopedia of Essential Medicinal Plants. Donetsk: Stalker. [In Russian].

Lemza, S. V., Linkhoeva, E. G., Toropova, A. A., Azhunova, T. A., and Petrov, E. V. (2014). Influence of Plant Remedy "glucovit» on the Energy Status of Organism in Experimental Diabetes Mellitus. Bulletin of the Buryat State University, 12, 12-15.

Leporatti, M. L., and Ivancheva, S. (2003). Preliminary Comparative Analysis of Medicinal Plants Used in the Traditional Medicine of Bulgaria and Italy. J. Ethnopharmacology 87 (2-3), 123-142. doi:10.1016/s0378-8741(03)00047-3

Letova, I. (2019). Diabetes Mellitus: The Best Recepies of Health. Saint-Petersburg: Dilia. [In Russian].

Li, S., Fan, T.-P., Jia, W., Lu, A., and Zhang, W. (2014). Network Pharmacology in Traditional Chinese Medicine. Evid Based. Complement. Altern. Med. 2014, 1-2. doi:10.1155/2014/138460

Lisitsyn, A. F., and Molodozhnikova, L. M. (1989). Phytotherapy: Eclectical Medicine: Collection of Medicinal Herbs.VDNKh USSR Centralized pavilion "Health" Subject of Public Speaking "Green Cosmetics, Medicinal Herbs and Sensible Nutricion” LK. Arnica, Moskow: VDNKh USSR Centralized pavilion Health. [In Russian].

Madić, V., Petrović, A., Jušković, M., Jugović, D., Djordjević, L., Stojanović, G., et al. (2021). Polyherbal Mixture Ameliorates Hyperglycemia, Hyperlipidemia and Histopathological Changes of Pancreas, Kidney and Liver in a Rat Model of Type 1 Diabetes. J. Ethnopharmacol. 265, 113210. doi:10.1016/j.jep.2020.113210

Mahendran, G., and Rahman, L. U. (2020). Ethnomedicinal, Phytochemical and Pharmacological Updates on Peppermint (Mentha $\times$ Piperita L.)-A Review. Phytotherapy Res. 34 (9), 2088-2139. doi:10.1002/ptr.6664

Makhlayuk, V. P. (1991). Medicinal Plants in Traditional Medicine. Saratov: Volga Book Publishing House. [In Russian].

Malgaonkar, M., Shirolkar, A., Murthy, S. N., and Pawar, S. (2016). Ayurvedic Plants with Antidiabetic Potential. Medicinal Plants-Recent Advances in Research and Development. Germany: Springer, 439-468. doi:10.1007/978-981-10-1085-9_19

Mashkovskii, M. D. (2002). Medicinal Agents Vol. 1. Moscow: Novaya Volna. [In Russian].

Matkovskaya, A. N., Trumpe, T. E., and Sokolov, S. I. A. (1988). Phytotherapy in Combination Therapy of Diabetes Mellitus: Prelection. Moscow: Central Institute for Advanced Medical Training Order of Lenin. [In Russian].

Maznev, N. I. (2005). Diabetes Mellitus and Obesity: Profilactics and Threatment with Herbs. Moscow: ASS-Center. [In Russian].

Maznev, N. I. (2014). Stop - Diabet! 500 Recepies and Useful Tips. Moscow: AST [In Russian].

Melik-Gusseinov, V. V., and Rekkandt, S. A. (2014). Phytotherapy. A Reference Book for Using Medicinal Plants in Traditional and Alternative Medicine (Monography). Volgograd: VolGMU Publishing house. [In Russian].

Micheli, L., Lucarini, E., Trallori, E., Avagliano, C., Caro, C., Russo, R., et al. (2019). Phaseolus vulgaris L. Extract: Alpha-Amylase Inhibition against Metabolic Syndrome in Mice. Nutrients 11 (8), 1778. doi:10.3390/nu11081778

Minaeva, V. G. (1991). Medicinal Plants of Siberia. Novosibirsk: Nauka. Siberian branch [In Russian].

Molokovskii, D. S., Davydov, V. V., and Khegai, M. D. (2002). Antidiabetic Activities of Adaptogenic Formulations and Extractions from Medicinal Plants. Rastitel'nye Resursy 38, 15-28. 
Mukherjee, P. K., Banerjee, S., and Kar, A. (2018). Exploring Synergy in Ayurveda and Traditional Indian Systems of Medicine. Synergy 7, 30-33. doi:10.1016/ j.synres.2018.10.003

Nagalievska, M., Sabadashka, M., Hachkova, H., and Sybirna, N. (2018). Galega Officinalis Extract Regulate the Diabetes Mellitus Related Violations of Proliferation, Functions and Apoptosis of Leukocytes. BMC Complement. Altern. Med. 18 (1), 1-13. doi:10.1186/s12906-017-2079-3

Namdul, T., Sood, A., Ramakrishnan, L., Pandey, R. M., and Moorthy, D. (2001). Efficacy of Tibetan Medicine as an Adjunct in the Treatment of Type 2 Diabetes. Diabetes Care 24 (1), 176-177. doi:10.2337/diacare.24.1.176

Nazina, Yu. V. (2006). Diabetes. The Best Recipes of Traditional Medicine from A to Ya. Moscow: OLMA media group. [In Russian].

Newman, D. J., and Cragg, G. M. (2020). Natural Products as Sources of New Drugs over the Nearly Four Decades from 01/1981 to 09/2019. J. Nat. Prod. 83 (3), 770-803. doi:10.1021/acs.jnatprod.9b01285

Nikolaychuk, L. V. (1997). Treatment of Diabetes mellitus with herbs. Minsk: The modern word, 256. [In Russian].

Nikolaychuk, L. V., and Zubitskaya, N. P. (2003). A 1000 Recepies for Patients with Diabetes Mellitus. Minsk: Interpresservis, 160. [In Russian].

Nikultseva, T. M. (1994). Phytotherapy: Collection of Folk Unconversional Methods of Treatments. Moscow: TWIC, 384. [In Russian].

Ninomiya, K., Matsuda, H., Kubo, M., Morikawa, T., Nishida, N., and Yoshikawa, M. (2007). Potent Anti-obese Principle from Rosa Canina: Structural Requirements and Mode of Action of Trans-tiliroside. Bioorg. Med. Chem. Lett. 17 (11), 3059-3064. doi:10.1016/j.bmcl.2007.03.051

Niu, H.-S., Liu, I.-M., Cheng, J.-T., Lin, C.-L., and Hsu, F.-L. (2008). Hypoglycemic Effect of Syringin from Eleutherococcus Senticosus in Streptozotocin-Induced Diabetic Rats. Planta Med. 74 (02), 109-113. doi:10.1055/s-2008-1034275

Okovitiy, S. V., Napalkova, S. M., Povydysh, M. N., Luzhanin, V. G., Goncharov, M. Yu., and Yakovlev, G. P. (2018). Medicinal Plants as a Source of Perspective Pharmaceutical Substances for Correcting Carbohydrate Exchange Disorders. Pharmacy 67 (7), 8-13. doi:10.29296/25419218-2018-07-0

Onipko, V. D. (2002). Comfortable Life and Diabetes Mellitus. Saint-Petersburg: Ves [In Russian].

Orhan, N., Aslan, M., Hosbas, S., and Deliorman, O. D. (2009). Antidiabetic Effect and Antioxidant Potential of Rosa Canina Fruits. Pharmacognosy Mag. 5 (20), 309-315. doi:10.4103/0973-1296.58151

Orlova, E. A. (2001). Phytotherapy: Medicinal Sacrament of Nature. Moscow: Terra - Book Club. [In Russian].

Osetrov, V. D. (1993). Alternative Herbal Medicine. Kiev: Naukova Dumka. [In Russian].

Osetrov, V. D., and Shreter, A. I. (2001). Herbalist for Women. A Practical Guide to Folk and Scientific Herbal Medicine and Homeopathy. Moscow: MSP Publishing House. [In Russian].

Panossian, A. G., Efferth, T., Shikov, A. N., Pozharitskaya, O. N., Kuchta, K., Mukherjee, P. K., et al. (2021). Evolution of the Adaptogenic Concept from Traditional Use to Medical Systems: Pharmacology of Stress- and Aging-related Diseases. Med. Res. Rev. 41 (1), 630-703. doi:10.1002/med.21743

Panossian, A., Seo, E.-J., and Efferth, T. (2018). Synergy Assessments of Plant Extracts Used in the Treatment of Stress and Aging-Related Disorders. Synergy 7, 39-48. doi:10.1016/j.synres.2018.10.001

Parasuraman, S., Thing, G., and Dhanaraj, S. (2014). Polyherbal Formulation: Concept of Ayurveda. Phcog. Rev. 8 (16), 73-80. doi:10.4103/09737847.134229

Pari, L., and Venkateswaran, S. (2004). Protective Role ofPhaseolus Vulgarison Changes in the Fatty Acid Composition in Experimental Diabetes. J. Med. Food 7 (2), 204-209. doi:10.1089/1096620041224120

Pavlić, B., Teslić, N., Zengin, G., Đurović, S., Rakić, D., Cvetanović, A., et al. (2021). Antioxidant and Enzyme-Inhibitory Activity of Peppermint Extracts and Essential Oils Obtained by Conventional and Emerging Extraction Techniques. Food Chem. 338, 127724. doi:10.1016/j.foodchem.2020.127724

Petkov, V. D. (1982). Modern Phytotherapy. Sofia: Medizina I. Fizkultura Publications House. [In Russian].

Pharmacopoeia Castrensis Rossica (1765). Military Pharmacopoeia Castrensis Rossica. $1^{\text {st }}$ Edition. Petropoli: Academia Scientiarum.

Pigulevskaya, I. S. (2018). Everything You Need to Know about Diabetes: An Essential Book for Diabetics: Types of Diabetes, Principles of Treatment,
Delicious and Healthy Dishes, Proper Exercises, Traditional Medicine, Herbal Medicine. Moscow: Centerpolygraph. [In Russian].

Pirogov, S. A. (2008). Secrets Of the Medicinal Species. Expirience Of a Work of Folk Phytopracticer. Barnaul: Altai printing plant. [In Russian].

Podduev, P. S. (2001). Medicinal Herbs and Traditional Methods of Treating Diseases: A Handbook. Bryansk: Bryansk Regional Printing House. [In Russian].

Popov, A. P. (2004). Doctor Book of Medicinal Herbs. Ekaterinburg: U-Faktoria. [In Russian].

Povydysh, M. N., Luzhanin, V. G., Ivkin, D. Yu., Belousov, M. V., and Yakovlev, G. P. (2018). Prospects for the Use of Phytotherapeutic Agents for Disorders of Fat and Carbohydrate Metabolism. Drug Dev. Registration (3), 130-135.

Protasenya, N. I., and Vasilenko, Yu. V. (1992). Medical Species (600 Prescriptions for the Treatment of the Most Common Diseases). Simferopol: Tavrida. [In Russian].

Register Russia (2021).On Line State Register of Medicinal Preparations by the Ministry of Public Health and Social Development of the Russian Federation. Available at: http://grls.rosminzdrav.ru/GRLS.aspx. [In Russian] (Accessed 2021).

Rendiuk, T. D. (2006). The way to the health. Herbal teas. Moscow: Drofa-Plus. [In Russian].

Ruzhenkova, I. V. (2014). Phytotherapy of Diabetes Mellitus: Medicinal Plant Treatment. Phoenix: Rostov on the Don. [In Russian].

Ryzhenko, V. I. (2007). Medical Species. Moscow: Onix. [In Russian].

Sabeva, N., Petkov, N., and Ogneva, V. (2004). Effect of Galega Officinalis Extracts on Glucose Homeostasis in Streptozotocin Diabetic Mice. Comptes RendusAcademie Bulgare des Sci. 57 (2), 95-98.

Safonov, N. N. (2016). Atlas of Medicinal Plants. 900 home Recipes that Will Help You Find health Gift Editions. Beauty and Health. Moscow: Publishing House 'E'.

Said, O., Fulder, S., Khalil, K., Azaizeh, H., Kassis, E., and Saad, B. (2008). Maintaining a Physiological Blood Glucose Level with 'Glucolevel', a Combination of Four Anti-diabetes Plants Used in the Traditional Arab Herbal Medicine. Evidence-Based Complement. Altern. Med. 5 (4), 421-428. doi:10.1093/ecam/nem047

Said-Shah, R. (2001). Spices that Heal. St. Petersburg: VES Publishing House.

Salehi, B., Ata, A., V. Anil Kumar, N., Sharopov, F., Ramírez-Alarcón, K., RuizOrtega, A., et al. (2019). Antidiabetic Potential of Medicinal Plants and Their Active Components. Biomolecules 9 (10), 551. doi:10.3390/biom9100551

Salehi Nowbandegani, A., Kiumarcy, S., Rahmani, F., Dokouhaki, M., Khademian, S., Zarshenas, M. M., et al. (2015). Ethnopharmacological Knowledge of Shiraz and Fasa in Fars Region of Iran for Diabetes Mellitus. J. Ethnopharmacol. 172, 281-287. doi:10.1016/j.jep.2015.06.017

Sammons, H. M., Gubarev, M. I., Krepkova, L. V., Bortnikova, V. V., Corrick, F., Job, K. M., et al. (2016). Herbal Medicines: Challenges in the Modern World. Part 2. European Union and Russia. Expert Rev. Clin. Pharmacol. 9 (8), 1117-1127. doi:10.1080/17512433.2016.1189326

Samylina, I. A., Ermakova, V. A., Bobkova, N. V., and Anosova, O. G. (2010). Pharmacognosia. T. 3: Medicinal Plant Raw Materials, Collections. Plant Powders. Medicines Based on Ground Plant Raw Materials. Moscow: GEOTAR-Media. [In Russian].

Sato, Y. (2004). The Treatment Strategy for Diabetes Mellitus by Kampo Medicine. Kampo Med. Nihon Toyo Igaku Zasshi 55 (6), 737-750. doi:10.3937/KAMPOMED.55.737

Seredin, R. M., and Sokolov, S. D. (1973). Medichinal Plants and Their Usage. Stavropol: Stavropol's Publishing house. [In Russian].

Shikov, A. N., Narkevich, I. A., Flisyuk, E. V., Luzhanin, V. G., and Pozharitskaya, O. N. (2021). Medicinal Plants from the 14th Edition of the Russian Pharmacopoeia, Recent Updates. J. Ethnopharmacology 268, 113685. doi:10.1016/j.jep.2020.113685

Shikov, A. N., Pozharitskaya, O. N., Kamenev, I. Yu., and Makarov, V. G. (2011). Medicinal and Spice Plants in Russia. Z. ArzneiఓGewurzplanzen 16, 135-137.

Shikov, A. N., Pozharitskaya, O. N., and Makarov, V. G. (2018). Challenges in the Investigation of Combinatory Modes of Action of Nutrients and Pharmaceuticals. Synergy 7, 36-38. doi:10.1016/j.synres.2018.10.002

Shikov, A. N., Pozharitskaya, O. N., Makarov, V. G., Wagner, H., Verpoorte, R., and Heinrich, M. (2014). Medicinal Plants of the Russian Pharmacopoeia; Their History and Applications. J. Ethnopharmacology 154 (3), 481-536. doi:10.1016/j.jep.2014.04.007

Shikov, A. N., Tsitsilin, A. N., Pozharitskaya, O. N., Makarov, V. G., and Heinrich, M. (2017). Traditional and Current Food Use of Wild Plants Listed in the Russian Pharmacopoeia. Front. Pharmacol. 8, 841. doi:10.3389/fphar.2017.00841 
Shojaee, S. S., Vahdati, A., Assaei, R., and Sepehrimanesh, M. (2015). Effect of Galega Officinalis Leaf Powder and Trigonella Foenum-Graecum Seed Powder on Blood Glucose Levels and Weight Gain in a Diabetes Mellitus Rat Model. Comp. Clin. Pathol. 24 (1), 145-148. doi:10.1007/s00580-013-1873-7

Sidorova, Y., Shipelin, V., Mazo, V., Zorin, S., Petrov, N., and Kochetkova, A. (2017). Hypoglycemic and Hypolipidemic Effect of Vaccinium Myrtillus L. Leaf and Phaseolus vulgaris L. Seed Coat Extracts in Diabetic Rats. Nutrition 41, 107-112. doi:10.1016/j.nut.2017.04.010

Sinyakov, A. F. (1992). About shoots and roots. Moscow: Physical education and sports, 272. [In Russian].

Sinyakov, A. F. (1999). Herbal Treatment. A Practical Guide. Moscow: JSC EKSMO-Press Publishing House. [In Russian].

Skalli, S., Hassikou, R., and Arahou, M. (2019). An Ethnobotanical Survey of Medicinal Plants Used for Diabetes Treatment in Rabat, Morocco. Heliyon 5 (3), e01421. doi:10.1016/j.heliyon.2019.e01421

Sklyarevsky, L. I. A., and Gubanov, I. A. (1989) Medicinal Herbs in Daily Graft. Moscow: Rosagropromizdat. [In Russian].

Smolianskii, B. L., and Lifliandskii, V. G. (2004). Diabetes Mellitus Treatment. Saint-Petersburg: Neva Publishing house. [In Russian].

Sokolov, S. Ya. (2000). Phytotherapy and Phytopharmacology: A Guide for Physicians. Moscow: Medical Information Agency. [In Russian].

Sokolov, S. Ya., and Zamotaiev, I. P. (1984). Guide to Medicinal Plants. Phytotherapy. Moscow: Medizina. [In Russian].

Stratton, C. F., Newman, D. J., and Tan, D. S. (2015). Cheminformatic Comparison of Approved Drugs from Natural Product versus Synthetic Origins. Bioorg. Med. Chem. Lett. 25 (21), 4802-4807. doi:10.1016/j.bmcl.2015.07.014

Suzuki, H., Sone, H., and Watanabe, K. (2017). Japanese Kampo Medicines for Diabetes Mellitus. Japanese Kampo Medicines for the Treatment of Common Diseases: Focus on Inflammation. Netherlands: Elsevier, 69-73. doi:10.1016/ B978-0-12-809398-6.00008-1

Xutian, S., Tai, S., and Yuan, C. S. (2014). Handbook of Traditional Chinese Medicine (Singapore: World Scientific). (In 3 Volumes).

Taghizadeh, M., Rashidi, A. A., Taherian, A. A., Vakili, Z., Sajad Sajadian, M., and Ghardashi, M. (2016). Antidiabetic and Antihyperlipidemic Effects of Ethanol Extract of Rosa Canina L. Fruit on Diabetic Rats. J. Evid. Based Complement. Altern Med. 21 (4), NP25-NP30. doi:10.1177/2156587215612626

Takács, I., Szekeres, A., Takács, Á., Rakk, D., Mézes, M., Polyák, Á., et al. (2020). Wild Strawberry, Blackberry, and Blueberry Leaf Extracts Alleviate StarchInduced Hyperglycemia in Prediabetic and Diabetic Mice. Planta Med. 86 (11), 790-799. doi:10.1055/a-1164-8152

Tarasenko, V. P., Ipatev, V. A., Shubin, V. A., Ivanina, E. V., Tarasenko, I. A., and Kholodilova, L. V. (1998). Modern Phytotherapy. Novosibirsk: The Science. Siberian enterprise RAS. [In Russian].

Tassa, B. D., Gogoi, G., and Das, S. (2012). A Comparative Study of the Hypolipidaemic and Antioxidant Activities of Ethanolic Extracts of Leaves of Phlogacanthus Thyrsiflorus, Oxalis Corniculata L. And Fragaria Vesca in Albino Rats. Asian J. Pharm. Biol. Res. 2, 12-18.

The State Pharmacopoeia of Russian Federation. (2018). Fourteenth ed. Moscow: Ministry of public health of Russian Federation. [In Russian].

Timmis, A., Townsend, N., Gale, C. P., Torbica, A., Lettino, M., Petersen, S. E., and De Smedt, D. (2020). European Society of Cardiology: Cardiovascular Disease Statistics 2019. Eur. Heart J. 41 (1), 12-85. doi:10.1093/eurheartj/ ehz859

Tokgöz, H. B., and Altan, F. (2020). Hypericum perforatum L.: a Medicinal Plant with Potential as a Curative Agent against Obesity-Associated Complications. Mol. Biol. Rep. 47 (11), 8679-8686. doi:10.1007/s11033-020-05912-7

Tong, X.-L., Dong, L., Chen, L., and Zhen, Z. (2012). Treatment of Diabetes Using Traditional Chinese Medicine: Past, Present and Future. Am. J. Chin. Med. 40 (05), 877-886. doi:10.1142/S0192415X12500656

Trofimenko, A. G., and Mogilny, N. P. (1998). Doctor Book. Moscow: JSC ASTV. [In Russian].

Turishchev, S. N. (2005). Phytotherapy for Everyone. Moscow: OLMAPRESS.Invest. [In Russian].

Turishchev, S. N. (2000). Rational Phytotherapy: Herbal Therapy. Moscow: Informpechat [In Russian].
Turova, A. D., and Sapozhnikova, E. N. (1989). Medicinal Plants of USSR and Their Applications. Moscow: Medizina [In Russian].

Ulrich-Merzenich, G. S. (2014). Combination Screening of Synthetic Drugs and Plant Derived Natural Products-Potential and Challenges for Drug Development. Synergy 1 (1), 59-69. doi:10.1016/j.synres.2014.07.011

Venkateswaran, S., Pari, L., and Saravanan, G. (2002). Effect ofPhaseolus Vulgarison Circulatory Antioxidants and Lipids in Rats with Streptozotocin-Induced Diabetes. J. Med. Food 5 (2), 97-103. doi:10.1089/109662002760178186

Vichkanova, S. A., Kolkhir, V. K., Sokolskaya, T. A., Voskoboinikova, I. V., and Bykov, V. A. (2009). Medicinal Products from Plants. Moscow: Scientific publication.ADRIS. [In Russian].

Vinogradova, T. A., GazhevVinogradov, B. N., Vinogradov, V. M., and Martynov, V. K. (2001). Practical Phytotherapy. Moscow: Valerie SPDEksmo-Press [In Russian].

Volynchenko, V. A. (2003). Medical Herbal Species: 3600 Healing Recipies. Donetsk Stalker Publishing house ACT. [In Russian].

Wang, J., Zhou, B., Hu, X., Dong, S., Hong, M., Wang, J., et al. (2021). Deciphering the Formulation Secret Underlying Chinese Huo-Clearing Herbal Drink. Front. Pharmacol. 12, 654699. doi:10.3389/fphar.2021.654699

Wouters, O. J., McKee, M., and Luyten, J. (2020). Estimated Research and Development Investment Needed to Bring a New Medicine to Market, 2009-2018. Jama 323 (9), 844-853. doi:10.1001/jama.2020.1166

Xiao, E., and Luo, L. (2018). Alternative Therapies for Diabetes: A Comparison of Western and Traditional Chinese Medicine (TCM) Approaches. Cdr 14 (6), 487-496. doi:10.2174/1573399813666170519103230

Xin, S., Jia, Y., and Huinan, Q. (2014). Research Progress and Prospect of Shennong Bencao Jing. Chin. Arch. Traditional Chin. Med. 32 (9), 2151-2154.

Xu, Y. X. Z., Xi, S., and Qian, X. (2019). Evaluating Traditional Chinese Medicine and Herbal Products for the Treatment of Gestational Diabetes Mellitus. J. Diabetes Res. 2019, 1-6. doi:10.1155/2019/9182595

Xue, J., Shi, Y., Li, C., and Song, H. (2019). Network Pharmacology-based Prediction of the Active Ingredients, Potential Targets, and Signaling Pathways in Compound Lian-Ge Granules for Treatment of Diabetes. J. Cel. Biochem. 120 (4), 6431-6440. doi:10.1002/jcb.27933

Yordanov, D., Nikolov, P., and Boychinov, A. S. P. (1972). Phytotherapy. Sofia: Medicine and Physical Education. [In Russian].

Zagayko, A. L., Chumak, O. I., Filimonenko, V. P., and Koshevoy, O. M. (2016). Investigation of the Effect of Dry Extracts from the Leaves of Cowberry and Bilberry Adding L-Arginine on Metabolic Parameters in Experimental Diabetes Type II. Ukr. Biofarm. Ž. 3 (44), 70-74. doi:10.24959/ubphj.16.43

Zeljković, Ć. S., Šišková, J., Komzáková, K., De Diego, N., Kaffková, K., and Tarkowski, P. (2021). Phenolic Compounds and Biological Activity of Selected Mentha Species. Plants 10 (3), 550. doi:10.3390/plants10030550

Zhang, J., Kang, M.-J., Kim, M.-J., Kim, M.-E., Song, J.-H., Lee, Y.-M., et al. (2008). Pancreatic Lipase Inhibitory Activity of taraxacum Officinale In Vitro and In Vivo. Nutr. Res. Pract. 2 (4), 200-203. doi:10.4162/ nrp.2008.2.4.200

Zmeev, L. E. (1896). Russkie Vrachebniki: A Study in the Field of Our Ancient Medical Writing. Saint-Petersburg: Typography by Lamakov, 276. [In Russian].

Łabuda, H., Buczkowska, H., Papliński, R., and Najda, A. (2017). Secondary Metabolites of Phaseoli Pericarpium. Acta Scientiarum Polonorum, Hortorum Cultus 16 (6), 187-200. doi:10.24326/asphc.2017.6.17

Conflict of Interest: The authors declare that the research was conducted in the absence of any commercial or financial relationships that could be construed as a potential conflict of interest.

Copyright $\odot 2021$ Shikov, Narkevich, Akamova, Nemyatykh, Flisyuk, Luzhanin, Povydysh, Mikhailova and Pozharitskaya. This is an open-access article distributed under the terms of the Creative Commons Attribution License (CC BY). The use, distribution or reproduction in other forums is permitted, provided the original author(s) and the copyright owner(s) are credited and that the original publication in this journal is cited, in accordance with accepted academic practice. No use, distribution or reproduction is permitted which does not comply with these terms. 